\title{
Childhood Determinants of Adult Psychiatric Disorder
}

\author{
Tom Fryers ${ }^{1,2}$ and Traolach Brugha ${ }^{2, *}$ \\ ${ }^{1}$ International and Public Health, School of Health Sciences, New York Medical College, USA \\ ${ }^{2}$ Department of Health Sciences, University of Leicester, UK
}

\begin{abstract}
The aim of this project was to assess the current evidence from longitudinal studies for childhood determinants of adult mental illness. Because of the variable and often prolonged period between factors in childhood and the identification of mental illness in adults, prospective studies, particularly birth cohorts, offer the best chance of demonstrating associations in individuals.

A review was undertaken in 2006 of the published literature from longitudinal studies, together with some large-scale retrospective studies and relevant reviews which provided supplementary evidence. The main focus was upon potentially ameliorable characteristics, experiences or situations of childhood; however, other factors, not determinants but precursors, associated with later mental illness could not be left out.

Seven major electronic data-bases of published research were interrogated with a range of key-words and the results supplemented from personal searches, enquiries and reference trails. In excess of 1,500 abstracts were read to select 250 papers for full review. The material was assessed in relation to ten factors:

Psychological disturbance; Genetic Influences; Neurological Deviance; Neuroticism; Behaviour; School Performance; Adversity; Child Abuse or Neglect; Parenting and parent-child relationships; Disrupted and Disfunctional Families.

In 2011 the search was repeated for the period 2006 to mid-2011, using the same search terms and supplemented in the same manner. Over 1,800 abstracts emerged and almost 200 papers selected for more detailed review. These were then integrated into the original text with modifications where necessary. The whole text was then revised and edited in January / February 2012.

There is continuing evidence for the association with later mental ill-health for each of these ten factors, but with different degrees of conviction. The evidence for each is discussed in detail and weighed both separately and in relation to others. These are then summarised, and the research implications are considered. Finally, the implications for prevention are discussed together with the practical potential for preventive and health-promoting programmes.
\end{abstract}

Keywords: Review, Epidemiologic Studies, Epidemiologic Factors, Cohort Studies, Association Studies, Genetic, Causality, Risk Factors, Socio-economic, actors, Confounding Factors, Epidemiology, Prevention, Humans, Child, Adolescent, Adult, Female, Male, Mental Disorders, Mood Disorders, Depressive Disorders, Schizophrenia, Substance -Related Disorders, Neurology, Neuro-behavioural Manifestations, Psychology, Child Behaviour, Adolescent Behaviiour, Adaptation, Psychological, Depression, Development, Personality, Abuse, Child Sexual, Neglect, Child, Stressful Events, Social, Support, Parenting, Families, Educational Achievement.

\section{PREFACE}

This project arose out of the continuing work in and through STAKES research institute in Helsinki on mental health indicators for use throughout the European Union. Indicators, in this context, are health related data-variables which are known to be associated in populations with real and important health problems, and which will permit monitoring in populations, an important aspect of public health.

Health problems include specific and well-defined serious diseases which give rise to serious morbidity or death and are often well recorded; common illnesses which are

*Address correspondence to this author at the Department of Health Sciences, University of Leicester, UK; Tel: +44 1162523211 ;

Fax: +44 116252 3272; E-mail: tsb@le.ac.uk inconsistently defined or recorded yet result in substantial loss of working days; disabilities which limit economic and social function; addictions and behavioural problems not always known to health agencies; psychological distress which may or may not be a normal reaction to adverse circumstances; and many other phenomena. Indicators may be records of disease recorded in health care systems; though these are often deficient for epidemiological purposes, they may still be useful as indicators, especially for comparisons over time. Disease registers and research may produce better epidemiological data for understanding aetiology and effectiveness of interventions, but which may also serve as indicators. However, other variables not directly related to disease processes or subjective ill-health may act as indicators, such as socio-economic measures, school attendance or work absence data, and community experience of civil or military conflict or natural disaster. 
Because of the pervasive tendency to ignore or minimise the impact of mental ill-health on populations, it is particularly important to encompass mental health in the indicators promoted across the EU for public health monitoring. The development of mental health indicators was the common context for seven linked projects arising out of previous work directed by STAKES, and recommendations for indicators arising from this study are to be found in the MINDFUL report [1].

Improving indicators, however, was not the only, nor indeed the principle aim of this particular project. It arose out of previous work by the first author on social inequalities in mental health $[2,3]$, and current concerns in the Department of Psychiatry in Leicester, and colleagues in the closely associated Medical School at Warwick [4]. It's principle aim was to explore the evidence available in the research literature that particular characteristics of childhood and childhood experience were determinants, that is, causes of later mental ill-health. It is better, of course, to speak of causal factors and causal processes, because rarely does one factor stand alone as causing illness, least of all mental illness. It is also necessary to be aware of other factors which may not be causes but are pre-cursors or early manifestations of what only later can we see to be a long-term mental-health problem.

In searching for evidence of such determinants, we were aware of a large existing literature including many reviews which generally examine retrospective and cross-sectional data which have grave limitations in pursuing long-term temporal relationships. So, given the inevitable limitations of time, we focussed largely upon longitudinal research, mainly large-scale birth-cohort studies, which have follow-up data through childhood and well into adult life.

Because we also have a practical interest in the possibility of intervention in countries of the EU to improve the lot of children and diminish the burden of mental illness in both children and adults, we gave the research an emphasis on ameliorable factors, in which intervention is at least theoretically possible. There may, as yet, be few programmes of proven effectiveness, but then, at least, there would be a case to be made professionally and politically for interventions to be tried and tested. It is hopeful that, at least in some countries, there are currently suggestions of interest at government level in doing something. Garnering the evidence already available should be of immediate use.

This is a review of epidemiological studies within the published literature, necessarily constrained by the methods, definitions, measures and chosen analyses of the studies and their published work. As in most fields, there is little published showing negative results, that is, in this context, showing no association between childhood factors and later mental illness, though some particular factors showing no association are often given within more general papers. There is, therefore, the risk of some bias. There is very little research on health-promoting or salutogenic factors and more work should be encouraged. In this review, it has not been possible, or, perhaps, appropriate, to enter much into psychological interpretation of childhood experience, such as trauma theory in relation to child abuse, or attachment theory in relation to parent-child relationships; these will be found in more appropriate texts.

\section{SECTION A: INTRODUCTION, BACKGROUND AND METHODS}

\section{Mental Illness and Children at Risk}

\subsection{Background}

In recent years there has been a great deal of professional interest in mental health research, including seeking possible causes of serious mental illness, mostly in adults. Most of this work has focussed upon proximal factors in adulthood necessarily using cross-sectional or short follow-up data, and easily known demographic, family and personal information. It has necessarily been dependent mainly upon retrospective data, commonly from relatively small samples or clinical series not representative of the general population.

From all this work an understanding has emerged of the 'cause' of serious mental illness as complex, varied and multi-factoral, encompassing elements of genetic constitution, childhood experience, characteristics of personality, significant life events, the quality of relationships, economic and social situation, life-style choices such as alcohol and other drugs, and aging. Some of these factors have been elucidated to the point of representing acknowledged risk factors for specific forms of mental illness or mental illness in general, such as familial genes, relative poverty, major trauma, excessive alcohol consumption, extreme negative life-events, poor education, and long-term unemployment.

These may all be experienced in childhood and we do not need research to tell us that poverty, inadequate education and life events such as loss of a parent or displacement as a refugee by war, or trauma such as child sex abuse are bad. Nor should it need evidence of later consequences such as mental illness to argue for the prevention of such situations and experiences. The strongest argument is in terms of human rights. However, the issues are not generally given a high priority and people may think them exaggerated or assume that these things are just part of human life and children get over them anyway. But we should not be willing to accept these as inevitably part of human life, but fight for a better life for our children - and hope thereby for a better life for adults and the whole community. And, though there is great diversity of reactions to adversity in children as in adults, and some victims of seriously adverse events or conditions in childhood do appear to survive intact, for very many it is not true that they 'get over' them, and many are, indeed, blamed for the experience of which they were the victim (for example rape, illiteracy, continuing poverty), or for not being able to 'get over' it.

In these circumstances we do need research to provide evidence of harmful effect, to highlight the issues, to keep them in the public eye, and to stimulate preventive action. Scientific evidence will give weight to compassionate argument and stimulate preventive action in childhood. The best evidence is likely to come from professionally evaluated programmes of prevention and health promotion; the relative paucity of such evidence currently makes it much more difficult to raise the political and social profile of the issues.

Of course, we also need epidemiological research relating risk factors to later illness, to understand the nature and origins of mental illness, whether in children, adolescents or 
adults, and to inform treatment and care of those who suffer. Although the starting point of this review, and the weight of data analysed relate to mental illness in adults, it is not in any way to be taken as ignoring the reality of mental illness in children and adolescents. This is likely to be related similarly to earlier experiences, may be related in a variety of ways to later mental illness, and is just as important to recognise, take seriously and treat appropriately.

\subsection{Childhood Variables; Characteristics, Situations and Experiences}

Although our focus was upon ameliorable factors, it proved impossible to ignore others, such as genetic factors, which cannot (as yet) be changed, but need to be taken into account in any programme. Moreover, factors in childhood which have been studied, and for which, therefore, there are data, are often not independent of each other; we must discriminate, for example, between child abuse, neglect, and inadequate parenting, for they raise different issues and may require different intervention, but there is clearly overlap in many cases, and in some the apparently different factors are really part of a more complex overall picture. However, it is necessary to separate them for research to be done at all, and this review was necessarily guided by the studies performed and published. Their likely inter-relationships need to be kept in mind throughout.

There are wider issues of inter-relationships which barely arise in the literature here reviewed, and which this study cannot, therefore, deal with. Persons are not primarily adults whose characteristics have been determined in childhood which is not important in itself but only as it affects them as adults. Indeed, for most populations during most of human history, what we call childhood, even for those that survived into adult life, constituted the greater part of life. We tend, perhaps, to diminish the status of being a child in populations with average life expectancies from birth of over 70 years, to a mere preparation for adulthood. An emphasis within schools, for example, of 'education for the world of work' reinforces this.

But in recent years there has been a growing understanding also of the complexity of the relationships throughout the whole life-course between experience, personality and illness. Adversities on the one hand, and positive features of childhood experience on the other, build up personalities with areas of both vulnerability and resilience, and, no doubt, experience continues to modify these throughout life. They, in turn, determine the response of individuals to particular experiences, adverse or otherwise, and sometimes this includes effects upon mental as well as physical health. For example, although physicians conventionally discriminate between the common mental disorders, anxiety and depression, (excluding psychotic illness) they frequently appear together and may best be understood as a varying individual response to experience, depending upon the resilience and vulnerabilities of that individual [5].

This review can enter very little into these areas. It is determined by the literature available and studied, and is thus forced to take the relatively simple epidemiological approach of examining defined 'risk factors' for later defined 'illness'.
Since most of the literature records illness in adults, that is the inevitable emphasis here.

Some of the variables studied have substantial evidence that they are associated with an increased risk of later mental illness - in childhood, adolescence or at any period of adult life, but conceptually they may not be determinants, that is not causal factors, but precursors, or early manifestations of mental illness. If the focus is upon mental illness in adult life, childhood mental health problems, the feature most strongly associated with later mental illness, can be viewed as a continuity of susceptibility and response to life's vicissitudes through at least later childhood and, possibly, all adult life. This in no way diminishes the importance of mental illness in children or adolescents, but it conceptualises a continuity of vulnerability to mental illness from childhood on. Of course, this continuity may not apply in all cases. It is equally possible for mental health problems in childhood and adolescence to affect future life chances (for example in relationships, education, work) which are associated with new problems leading to mental illness. In such cases, childhood mental health problems may indeed be determinants.

Other variables are probably not determinants but are indicators of underlying problems which might or might not be true determinants - for example, neurological deficits in childhood, which may cause mental health problems or may be part of a wider picture of cerebral damage or disorder with common earlier causes.

Some may very well be true determinants, but require other factors for expression - for example, certain gene combinations which will only cause a problem if particular conditions arise, an expanding current interest as knowledge of gene-environment interaction increases. Some, also true determinants, may contribute to an increased risk of later mental illness, but only to a very small degree if examined separately; however, in combination with others, with which they will frequently be associated, they may be part of an important causal process - for example the individual measured factors which constitute 'multiple childhood disadvantage'.

Of course, factors in childhood which are demonstrably causal may permit us to prevent later childhood mental illness as well as that reported in adults.

The relationships between these various types of childhood factor are complex and difficult to disentangle. In many cases we cannot know exactly what sort of relationship childhood experiences have with adult experiences. We are limited to the data available and the types of study published. We can only be relatively sure, where the data permit even of this, of epidemiological associations, that is statistical associations in populations, some of which give us some confidence of causal relationships, and some hope of preventive action. Overall, and simplified, the causal processes can be seen as combining personal vulnerability and precipitating factors [5]. Vulnerability for the young adult, perhaps built up in childhood by the operation of many factors, may be perceived as the susceptibility to react in certain ways to the somatic, inter-personal, physical and socio-economic stresses he or she will face throughout life, though there is no reason to believe that such susceptibility is not itself modified and varied throughout adult life. Vulnerability at the 
transition from child to adult is a key issue, and, therefore, factors in childhood which affect vulnerability in adolescence should be clarified as much as possible.

\subsection{Epidemiological Research; Birth Cohorts and other Longitudinal Studies}

Because of the time interval involved, there is a major interpretative problem in relating features of childhood experience to adult mental illness, if research is reliant upon data from cross-sectional, short clinical follow-up and retrospective studies. In particular, the reliability of recall of childhood experience is a major source of uncertainty. The scientific literature, in so far as it is dependent upon such studies, cannot readily provide definitive results with a very variable time interval which could be anything from 10 to 50 years later.

Despite the huge problems involved in funding and managing long-term prospective studies through childhood well into adult life, there is now a surprising number of major birth-cohort studies, some with very large national representative samples, which provide potential for firm linkage between observations made in childhood and later clearly defined mental illness. However, their potential is not realised with regard to these specific issues unless appropriate data were collected at particular ages, now long since past. Sadly, relevant data were often not collected at all, and data collected were not always of the highest quality by present-day standards. At the time of their inception (for example, the British 1946 and 1958 birth cohorts) and during their followup in childhood, many issues now considered important were simply not thought of, for example the quality of parenting.

Moreover, the practical and financial limitations of each wave of data collection required difficult decisions about what data could be collected, and these decisions were not necessarily what we would have hoped for now. Even had they wanted to collect mental health data more thoroughly, there were no adequate validated measures until the 1980s, so measurement precision in the longest-standing cohorts is generally much less than we now expect. Newer cohorts have the advantage of these developments, but have not yet the length of follow-up. And, in practice, none can collect all the data all researchers would desire.

With changing research priorities, even the cohort studies are dependent for some data now considered important, on retrospective questionnaires. For example, the 1946 birth cohort only collected data on parenting style and quality at age 43 , which raises the same questions of reliability of retrospective self-report data as in cross-sectional studies. The later British cohorts were able to collect some relevant data during childhood, but mostly during adolescence. Recall might be very good, but bias is certainly possible at a time of changing relationships between child and parent. With all the uncertainties in measurement of parental relationships, some mis-classification is inevitable. All these methodological limitations of cohorts tend to diminish their capacity to show differences, thus minimising positive results.

The measures of mental health outcomes at later ages are likely to be more robust and validated, being based on sound epidemiological experience and using more recently collected data. Yet no measure is perfect and none is without ambiguity in discriminating mental health problems. They vary in important respects, particularly with regard to the period of time for which the data are requested. The chosen instruments tend to be those which are simply and easily applied and analysed, such as the Malaise Inventory and the 12-item General Health Questionnaire; truly diagnostic data can rarely be collected on such numbers with the resources available, or with the co-operation of a large sample. However, in some countries cohort data can be linked to thorough national, regional or institutional records and registers, sometimes including verified standardised diagnoses. Even then, analytical dichotomies necessarily ignore the realities of gradations of illness and overlapping diagnostic categories. There are, therefore, many things which tend to reduce the capacity to demonstrate significant relationships, thus minimising positive results.

Although birth cohorts have usually been selected to represent a national population, they cannot do so perfectly, and representativeness tends to diminish in successive followups, as different groups drop out differentially. On the whole the British cohorts have tended to be biased towards nonmanual classes, two-parent families and female subjects. Representativeness is also problematic in smaller cohorts, such as the highly productive New Zealand cohorts, or where only reduced samples are surveyed in later waves, such as in the UK 1970 cohort in 2000.

Many possible confounding variables can be found in cohort data, but the most important is likely to be social class, or socio-economic group. Most characteristics, experiences and situations of childhood are profoundly affected by differences in social and economic circumstances, and some have suggested that these are the key determinants of variables such as parenting style and quality. However, though the importance of social and economic circumstances is incontrovertible and must always be accommodated in analyses, a great deal of research over many years has shown the other variables of childhood to have independent associations with mental ill-health both in adolescence and later adult life (see the literature review in [6])

It should also be recognised that cohort studies followed through from at least early childhood into adult life are necessarily recording childhood experienced some time ago, in some cases a long time ago. They are inevitably somewhat 'out of date' with respect to the economic, social and cultural context of childhood which will be very different for children 'now'. Cross-sectional studies offer a significant advantage when they record current events or very recent experiences, and can generate new ideas and hypotheses. Where possible, recent data from some such studies are included in this review.

However, in spite of these many problems of cohort research, they remain the best hope of elucidating childhood factors which contribute to the risk of adult mental illness, and which might be susceptible to intervention in order to reduce the risk. And much published material gives justification for that hope.

\section{Aims, Methods and Issues of Interpretation}

\subsection{Summary of Aims}

The main aim of this study, therefore, was to review the evidence currently available linking childhood factors to the 
Table 1. Original Searches, 2005

\begin{tabular}{|c|c|c|c|c|c|}
\hline Database & Date Range & Search Date & Hits. & Filename & BRS \\
\hline $\begin{array}{l}\text { Medline } \\
\text { (ovid) }\end{array}$ & 1966-date & $6 / 10 / 05$ & 873 & endnotemedline.txt & endnotetom \\
\hline $\begin{array}{c}\text { PsycInfo } \\
\text { (Ebsco) }\end{array}$ & 1887-date & $12 / 10 / 05$ & 276 & ebsco $123 \& 4$ & 3endnote \\
\hline $\begin{array}{l}\text { Embase } \\
\text { (Ovid) }\end{array}$ & 1980-date & $6 / 10 / 05$ & 472 & endnoteembase.txt & endnotetom \\
\hline Sci citation index (WOK) & 1970-date & $11 / 06 / 05$ & 294 & $\begin{array}{l}\text { sci9905.txt } \\
\text { sci8698 }\end{array}$ & 2endnote \\
\hline Soc Sci Cit index (WOK) & $\begin{array}{c}1970-95 \\
\text { 1996-date }\end{array}$ & $12 / 10 / 05$ & $\begin{array}{c}72 \\
365\end{array}$ & $\begin{array}{c}\text { ssci197095.txt } \\
\text { ssci9605.txt }\end{array}$ & 3endnote \\
\hline Assia (CSA) & 1965-date & $11 / 10 / 05$ & 57 & assia.txt & 2endnote \\
\hline Cochrane Library (Wiley) & All & $12 / 10 / 05$ & $\begin{array}{l}1 \\
4\end{array}$ & cochrane sys reviews central & 3endnote \\
\hline
\end{tabular}

frequency of mental illness in adults. This would largely use evidence from prospective cohort studies, would focus mainly on factors that might be amenable to individual or population intervention to prevent mental disorder and promote mental health, and would have an emphasis on relevance to European and similar populations.

\subsection{Overall Plan for Literature Review}

Literature searches using key-words have been greatly facilitated in recent years by computer-accessed data bases, but searching is not always easy or effective for multidisciplinary topics in which key-words are poorly defined. This was the situation in this project. It was necessary, therefore, to perform multiple searches using a variety of keywords, and accessing several publication data banks relating to all medical and social sciences. This inevitably generated great numbers of abstracts to peruse in order to select relevant papers.

The general review plan undertaken was discussed and agreed within the research group, and was as follows:

- Scoping review of samples of literature generated by general searches.

- Determining the practical boundaries of the project.

- Determining the determinants and outcomes for full searches.

- Choosing the main search strategy and key-words required.

- Systematic search of all relevant data-bases.

- Review of all abstracts to select full papers for study.

- Analytical study of selected papers.

- Collation of published evidence.

- Assessing potential for further research.

In 2011 this was exactly repeated from the 'Systematic search' point, with new data being integrated into the 2006 text, and the whole text revised.

\subsection{Search Strategy and Literature Processing}

Features of childhood, and mental health outcomes are both characterised by ambiguous and varied definitions, varied and inconsistent measures, and inconsistent and inadequate research design. There are several relevant data-banks of published papers including general medical research, psychiatry and psychology, social sciences, and evidence-based reviews. Each have their own systems of search terms, and each will reveal a somewhat different range of papers relevant to a particular search, though overlapping to a varying degree.

To identify published work relating a range of possible childhood determinants to a range of adult mental disorders, the search strategy is, therefore, complex. The Table $\mathbf{1}$ below shows the seven data-banks of published research papers interrogated, and the number of 'hits' - possible relevant papers identified - produced by each.

This produced 2,414 references, mostly with abstracts for review, using Reference Manager software, from which to select those which appeared, from the abstract, to offer possible relevance to the current project.

In addition, two other sources of possible papers were used. First, the major large-scale long-term cohort studies are well known and papers published exploiting their data-sets may be catalogued and available on web-sites or from study leaders. Consultations were undertaken where possible with leaders of major cohort studies in Finland and the UK, and a wide range of people from many countries concerned with similar research. These long-term studies have been exploited chiefly by a limited range of researchers, and the literature data-banks have been searched specifically by their names.

Second, references in papers eventually obtained provided yet more papers suitable for review. In some cases, books and unpublished research reports, which do not generally appear in the data-bases, were obtained directly from authors or their institutions. All these strategies produced additional references. Thus in excess of 2,500 abstracts were 
Table 2. Re-run of Searches April / May, 2011

\begin{tabular}{|c|c|c|c|c|}
\hline Database & $\begin{array}{c}\text { Date } \\
\text { Range }\end{array}$ & $\begin{array}{c}\text { Date } \\
\text { search }\end{array}$ & 'Hits'. & No. of 'hits' in 2006 \\
\hline $\begin{array}{l}\text { Medline } \\
\text { (ovid) }\end{array}$ & Oct 2005-date & $4 / 4 / 11$ & 1144 & 843 \\
\hline $\begin{array}{l}\text { PsycInfo } \\
\text { (Ebsco) }\end{array}$ & Oct 2005-date & $23 / 5 / 11$ & 145 & $2761887-2005$ \\
\hline $\begin{array}{l}\text { Embase } \\
\text { (Ovid) }\end{array}$ & Sept 05 -date & $4 / 4 / 11$ & 196 & $472(1980-05)$ \\
\hline $\begin{array}{l}\text { Sci. Citation index } \\
\text { (WOK) }\end{array}$ & $\begin{array}{l}\text { 2006-date } \\
\text { Sept-Dec } \\
2005\end{array}$ & $\begin{array}{l}5 / 4 / 11 \\
5 / 4 / 11\end{array}$ & $\begin{array}{c}378 \\
14\end{array}$ & $294(1970-05)$ \\
\hline $\begin{array}{l}\text { Soc. Sci. Cit index } \\
\text { (WOK) }\end{array}$ & $\begin{array}{l}\text { Sept-Dec05 } \\
\text { 2006-date }\end{array}$ & $\begin{array}{l}5 / 4 / 11 \\
5 / 4 / 11\end{array}$ & $\begin{array}{c}26 \\
466\end{array}$ & $437(1970-05)$ \\
\hline Assia (CSA) & $\begin{array}{l}\text { Sept 2005- } \\
\quad \text { date }\end{array}$ & $5 / 4 / 11$ & 45 & $57(1965-05)$ \\
\hline $\begin{array}{c}\text { Cochrane } \\
\text { Library (Wiley) }\end{array}$ & Sept 2005-date & $23 / 5 / 11$ & $\begin{array}{c}0 \text { syst. reviews } \\
1 \text { 'dare' } \\
15 \text { 'central' }\end{array}$ & $\begin{array}{c}1 \text { syst. reviews } \\
4 \text { 'central' }\end{array}$ \\
\hline
\end{tabular}

reviewed, and from these sources, papers were selected for reading and evaluation.

Papers for full evaluation fell into four groups: 52 were reviews of relevant topics; 155 related to particular prospective studies, our main focus. In addition, there were 83 papers related to retrospective studies, some of which (especially very large samples) were also used for additional evidence, as also several papers out of 13 identified, which were concerned with preventive programmes, including some evaluations. Approximately 250 selected papers were obtained, read, relevant data extracted and collated in relation to ten variables, and the evidence of association with adult psychiatric disorder evaluated.

In 2011 the same systematic research was undertaken, (Table 2) with the results below.

\section{Re-run of searches April / May, 2011}

Similar further less formal searches were undertaken as in the first review to add to the 2,430 identified in the above search, so that, again, in excess of 2,500 abstracts were appraised. It is interesting that in the 5-6 years 2005-2011, more papers were identified than in the previous search (2384) using the same search terms but historically barely restricted. It is clear that there has been a substantial increase in publications in this field, particularly relating to child adversities and child abuse. Out of the recent searches, approximately 200 papers were selected for more detailed review. Because there are many fields covered, it was important to search also in less formal ways and follow up reference trails and leads from one field to another, to minimise gaps.

Ideally, this process would be duplicated by two independent reviewers who would then discuss and agree any differences in choice, but this was simply impracticable given the amount of work it demanded. However, the first author's draft was reviewed by the second author and ad- justments made to the treatment of papers where appropriate and after discussion. The narrative review method was used throughout; any more formal data synthesis was precluded by the heterogeneity of data which applied to all aspects of both determinants and outcomes. This has commonly been found even in reviews of more restricted, focussed topics than this $[7,8]$.

\subsection{General Points to Consider in Understanding and In- terpreting these Findings}

\subsubsection{Duration of Studies}

Evidence from longitudinal studies, especially birth cohorts are often limited to early adult outcomes, but increasingly they now reach middle-age. So the links with childhood, previously predominantly for mental ill-health in early adult life, are being extended.

\subsubsection{Probabilities Apply to Populations}

These are epidemiological studies so the results are statistical probabilities and proportions, relative to control groups or the general population. No such risk can be applied to any individual. It should be remembered that, in most cases, of children who experienced a particular adverse event or circumstance, or who revealed a particular disadvantageous characteristic in childhood, a majority do not apparently suffer serious psychiatric disorder in adult life. This is illustrated by Skodol and Bender [9] in an unusual study in which positive experiences of childhood were associated with significant remission of personality disorder diagnosed in early adult life; the more positive features of childhood, the better the prognosis.

\subsubsection{Discriminated Variables are not Necessarily Inde- pendent}

Most cohort studies encompass many variables, even where individual published papers focus upon only one or 
two. The evidence is rarely very specific for particular variables as measured, or particular outcomes as measured. Frequently a paper lists several, sometimes many variables, which show associations of varying degrees with adult psychiatric disorder, and subject their data to multi-variate analysis, or control and adjust for many variables to focus upon one or two. This simplifies the results but does not necessarily accurately reflect reality where these variables may inter-relate and even overlap. Their discrimination as separate variables is to some extent a convenient abstraction, and they do not necessarily represent distinct phenomena.

Therefore the variables described below individually must not be assumed to be independent. They are not the only factors, nor, necessarily, are they even the most important; we can only say that there is evidence that they antedate, predict, and might be involved in the causation of adult illness. But almost certainly they are part of a group of interrelated variables and part of a life-course process which we hope ultimately to understand, and which might explain some of the complex aetiology of mental illness in adults only 'some' because factors in adult life are undoubtedly also involved. And there is now no doubt that both genetic and environmental factors are involved, that they may, for any particular outcome, be inter-linked, and that interaction may be modified by other factors [10].

The choice of variables by particular researches will reflect many personal and local factors, rarely described, and one piece of research may influence others to examine the same variables. other variables may be neglected for no good reason. It is also true of this literature, as most others, that positive results are more likely to be reported that negative results. So some publication bias and research-emphasis bias are both inevitable - a review like this one is entirely dependent upon what is published and available.

The picture is undoubtedly complex, yet researchers must simplify in order to measure, analyse and interpret. Recent developments in statistics and computing have made complex analyses easier for the generality of researchers, but this does not facilitate the definition, measurement and collection of the complex data themselves, nor the interpretation of results. Indeed, the very facility of computer analysis may distort the results by giving apparent significance to unimportant elements, and distract the researcher, and the reader, from the main results.

\subsubsection{Measurements in Childhood are Often not Precise}

With few exceptions, the childhood variables measured are not precise; for example the measurement of 'neurotic' features of personality depends upon the validity of the construct underlying the measure, as well as the measure's accuracy in representing that construct and its capacity in practice to record observations consistently. Childhood 'neuroticism' measured thus has often been found to be associated with adult depression and anxiety, but some have questioned whether what is measured is anything more than a 'base level' of symptoms of depression and anxiety in the child, an early manifestation of what is more readily recognised later as a mental health problem. Other variables pose similar problems, and may be measured in a variety of ways which are not easily compared between different studies.
2.4.5. Different Measures of Psychiatric Disorder may Simply Different Constructs

Although there are now several well validated measures of psychiatric disorder in adults, the earliest birth cohort studies, for example the UK 1946 cohort, did not have the benefit of these for their earlier adult studies. Longitudinal studies covered by this review used a wide range of measures for a variety of practical reasons. These range from a few simple questions to adults recalling their experience of depression, through formal psychiatric diagnosis by trained psychiatrists, to repeated measures using a standard instrument. Inevitably, they measure different phenomena and must be interpreted as different constructs of illness.

\subsubsection{Lower-Order Taxonomic Divisions may be Spurious}

The now conventional and almost universal divisions of mental disorders within the standard classification systems (currently ICD-10; DSM-IV) have been immensely important in clarifying the diagnostic process in clinical work and facilitating the prescription and evaluation of treatment. But outside the practical demands of the clinic, it is not clear that the lower-order divisions represent separate phenomena. The most obvious example is lesser forms of depression and anxiety which are frequently found together. It is misleading to call this 'co-morbidity'; epidemiological evidence suggests that they may well be variations of the same general phenomenon, affecting different people in different ways [5]. For this reason they are now often called, collectively, 'the common mental disorders' [3].

To a lesser extent there are similar problems related to terms and categories such as 'major depression', 'psychotic depression', and 'uni-polar affective psychosis', or 'schizophrenia', 'schizophreniform disorders', and 'other nonaffective psychoses'. It should be born in mind that these are extremely useful constructs, but, in the absence (in most cases) of related pathology, they are not perfect descriptors of the disease processes involved. In the literature here reviewed, adult mental disorder is recorded and measured in many different ways, often generalising rather than using specific diagnostic groups. A common exception is schizophrenia, so this is often dealt with separately in the review. Published analyses discriminating other major groups such as serious or psychotic depression, or alcohol disorders, were not sufficiently common across the literature to permit more than generalisations in the review. More subtle measures, such as 'At risk mental state for psychosis' (ARMS) were not generally encompassed.

\section{SECTION B. CHILDHOOD FACTORS AND EVI- DENCE FOR THEIR IMPACT ON ADULT MENTAL HEALTH}

\section{Psychological Disturbance in Childhood and Adolescence}

\subsection{General Review}

In spite of measures used being many and varied, probably the most evidenced predictor of psychiatric disorder in adult life is psychological disturbance, psychiatric symptoms or diagnosable psychiatric disorder during childhood and adolescence. There is strong evidence for this continuity of disorder from many individual studies, not least the big co- 
hort studies. For example, Reef et al., [11-14] followed 2,076 children for 24 years and examined many facets of children's mental health; the chief predictors of adult mental illness were anxious children, oppositional defiant children and those with conduct disorders. But their main conclusion was a general one - there is substantial continuity of psychopathology. Anxiety and depression, delinquent and aggressive behaviour were 'core predictors' but the putative outcomes were not specific.

There are suggestions that anxiety/depression commencing in childhood or adolescence may be a different entity from adult-onset depression with or without anxiety, with different childhood associations. This has been explored recently by Dekker et al., [15] who used a Dutch longitudinal study encompassing several cohorts $(\mathrm{n} 2,976)$ to look at a variety of early life trajectories of depressive symptoms from age 4 to age 18 . They found significant differences for several groups, and particularly between boys and girls. Only in girls was a chronic trajectory of early childhood-onset depression identified, other trajectories showing peak ages and later improvement.

Depression has only been generally recognised in children since the 1970s [16] and there is still much debate about measurement in surveys and the clinical significance of the results. Depression can be found in younger children, but there is a sharp increase in adolescence [17]. Kandel and Davies in 1986 [18] found that depressed mood in adolescence predicted depression in adults, and there is now a substantial body of literature that, in spite of the many differences of measurement and interpretation, provides strong evidence for a number of temporal relationships [19]. It confirms that the strongest relationship of any childhood variable with adult depression is childhood or adolescent anxiety and depression; [16, 20-25].

For example, in the Christchurch (New Zealand) birth cohort, the strongest association was between diagnosed mood disorder in children and diagnosed mood disorder in adults [26]. However, the numbers involved beg questions about the categories used: at age 15 about $25 \%$ of the cohort met DSM III criteria for mental disorder, more in girls than boys, and predominantly anxiety and depression. At age 18, about $40 \%$ met the criteria, though less than a quarter of these had received treatment.

In this cohort, adolescent depression carried a risk of very early (by age 21) adult depression of three times that of others, and of anxiety twice that of others (figures adjusted for confounders). This translates into nearly two-thirds of adolescents at age 14-16 with depression experiencing a further episode of depression by age 21 , and half experiencing an episode of anxiety by 21 [16].

Recent studies confirm previous work, that anxiety/depression (closely related to 'internalising problems'), as well as conduct disorder, ('externalising problems') in adolescents, not only predict adult depression, but that, like earlier childhood mental health problems, its power to predict adult depression does not weaken with time [25]. A British 20-year follow-up of 149 children, mostly adolescent, with rigorously diagnosed major depressive disorder (MDD) found a recurrence rate of over $60 \%$ for MDD and over $75 \%$ for any depressive disorder $[27,28]$. In another recent study, about $75 \%$ of adults with depression had had depression also during adolescent years [29].

The Finnish 1981 Birth Cohort (n 5,346) has recently produced interesting data on the different expectations or risks for boys and girls, followed up to age 24 [30]. The most important single predictor of psychiatric admission to hospital was conduct disorder in boys and emotional problems in girls, though the combination of both childhood diagnoses was the strongest predictor in both sexes. The commonest diagnosis on admission was substance abuse disorder in young men and non-psychotic mood disorder in young women. But there was a lot of overlapping of diagnostic groups, and the relationships appear rather non-specific, a theme repeated in many studies.

An American study following up 354 children from age 5 reported that 'internalising problems' at age 15 were associated with about twice the expected frequency of major depression between ages 18 and 26 [31]. In the Netherlands, anxiety/depression in adolescence predicted similar disorders 10 years later $[17,23,32]$. The first British Birth Cohort, following over 5,000 births from 1946, shows persistent child affective disturbance (from teachers' questionnaires) as a powerful predictor of persistent adult affective disorder (OR 7.8) [33].

The effect of this continuity of morbidity should not, however, be exaggerated. In the 1958 British Birth Cohort [34] found that 'psycho-social problems' measured at age 16 were highly predictive of depression at the age of 33 (OR 2.9 for women; 2.3 for men) Continuing follow-up with a biomedical survey of 9,377 members of the original 17,416 births, to age 45 (54\% of survivors) the Malaise Inventory (MI) was used to measure psychological distress at ages 23, 33 and 42 years. Both internalising and externalising problems had been measured at ages 7 and 11 with the teacherrated Bristol Social Adjustment Guides, and at age 16 with the Rutter Scales. They did find that both types of child problem gave an increased risk of 1.5 to 2 times for adult psychological ill-health at all subsequent ages, but most respondents with psychological ill-health in childhood, or in early adulthood, did not have a psychiatric diagnosis at age 45: only $8 \%$ and $17 \%$ respectively. On the other hand, risk of mid-life disorder increased significantly with the number of adult experiences of psychological ill-health [35].

When matching child and adolescent diagnoses with adult illness the problems inherent in diagnosis are exaggerated, so that determining the degree of specificity in relationships is difficult. A recent paper from the Great Smoky Mountains Study, a longitudinal study of the development of psychiatric disorder in a representative sample of children from 11 counties in western North Carolina, followed up 1,420 children aged 9, to ages 16,19 and 21. They found "generalised anxiety and depression cross-predicted", but such a conclusion assumes that they are separate entities at any age, and it is much more useful to consider them as one, with a variety of expressions. Other patterns of relationship across ages were non-specific, which many others have found. Their data suggested that oppositional defiant disorder in childhood or adolescence was a precursor of many later disorders - though they only had data to age 21 [36]. 
Suicidal ideation, attempted suicide, and successful suicide are closely related to experience of depression or other psychological disturbance. A review in 2000 [37] could say "Current research evidence suggests that the strongest risk factors for youth suicide are affective disorders, substance use disorders, anti-social behaviour, and a history of psychopathology.". Of 149 British adolescents with MDD followed up for 20 years, $2.45 \%$ had committed suicide, six times the expected rate, and the risk of suicide attempt at least once was over $44 \%$, higher still where MDD and Conduct Disorder co-existed [27, 28]. In the Christchurch cohort, mental health problems at ages 14, 15 and 16, especially mood disorders, were the strongest predictors of suicidal behaviour [38]; over a quarter reported suicidal thoughts by age 21 , over five times expected rates; $8 \%$ reported an attempt, twelve times expected rates [26].

To keep this in perspective it should be noted that most of their children and adolescents with depression did not attempt suicide, and half of them reported never having had a suicidal thought. Indeed, most adolescents with depression do not develop depression as young adults [25].

However, in cohort studies, anxiety/depression in children or adolescents also has associations with other adult outcomes, though they are much less studied. These include substance abuse, generally poor psychological functioning, and bi-polar disorder [39]; impaired social functioning and low academic achievement, nicotine dependence, employment problems and early parenthood [16]. The researchers from Christchurch stated that "Adolescent depression is a marker for long-term adjustment problems, but there is possible confounding with family characteristics, child adversity, child abuse, and neuroticism. Indeed, and for example, adolescents with depression were much more likely to have suffered child abuse or parental change [16]. It is hardly surprising that childhood or adolescent depression and anxiety should have antecedents of adversity and suffering; it offers possible opportunities for primary prevention but does not diminish the importance of treating the conditions when found.

Interpretation is also rendered more difficult by the finding of Patton et al., in 2003 [40] in Victoria, Australia, that the relationship between depression and life events within the period of adolescence, appears to be reciprocal, each modifying the risk for the other at the next stage. They suggest that features of personality or long-term adversity might underlie this phenomenen.

The variables that may confound analysis may, in fact, be important precursors of anxiety/depression in children and adolescents, possibly part of the long-term process of development of adult mood disorders. From reviews, the risk of childhood MDD appears to include parent psycho-pathology, family conflict, inadequate peer support and poor coping skills, but in a rather non-specific way [39]. The Christchurch cohort have found child mental health problems associated with socio-economic adversity in the family, marital disruption (mostly divorce or separation), poor parent-child attachment, exposure to child sexual or other abuse, neuroticism, and 'novelty-seeking' as an adolescent [38]. The researchers interpreted their findings as early adversities' associations with adult psychiatric disorder being 'mediated' by mental health problems in childhood and adolescence.
In the Dunedin (New Zealand) cohort, early mental health and behaviour problems, appeared strongly to predict psychiatric disorder at age 15, which in turn overwhelmingly predicted psychiatric disorder at age 18 [41]. In the Finnish Birth cohort of 1981, precursors of psychiatric disturbance at age 18 included not living with two biological parents at age 8 , and poor school achievement, as well as psychological or emotional problems subject to psychiatric consultation, or reported by parents, teachers or the child [42, 43]. It is also important to know if 'psychological problems' in children are persistent. The three-year follow-up of a sample of 2,587 children from the 1999 British Child and Adolescent Mental health Survey had data from parents, teachers and young people at base-line and follow-up using appropriate versions of the Strengths and Difficulties Questionnaire [44]. Latent mental health scores showed strong stability over time, suggesting that children with problems truly need help as they are unlikely to get better spontaneously. Poorer outcomes were related to (at base-line) externalising symptoms, reading difficulties, single-parent or re-constituted family; and (at follow-up) parental separation, parental mental illness, child illness, and loss of a close friendship. The authors comment that all these factors could be targeted in public health or clinical interventions.

In summary, there is evidence of pathways from psychological problems in early and mid-childhood, to psychological symptoms and well-defined disorder in adolescence, to psychiatric disorder in adults. However, much of the evidence up to now has not been very precise, and a recent exploration of the utility of psycho-pathological screening at ages 5 and 14 in the large Queensland cohort study concluded that the results did not justify a screening programme [45]. It is possible to infer from other work, including that from Britain above, that detection of risk-increasing life events might permit help to be offered to reduce the recurrence and severity of later disorder in those individuals.

One set of unusual studies in the USA focussed on specific panic disorder, using a sample of 1018 adults assembled for other purposes by Biederman et al., [46]. 58 persons were diagnosed with panic disorder which appeared to have a special high-risk relationship with a childhood history of separation anxiety, social phobia and simple phobia.

\subsection{Psychoses}

There has been a lot of work examining the childhood precursors of schizophreniform disorders, which conventionally have an onset in adolescence or early adulthood. There is generally consistent evidence that children who later develop schizophrenia commonly show behavioural disturbances and psychopathology, intellectual and language deficits, and early motor delays (see Section 5). However, it is now becoming increasingly clear that children do also sometimes show frankly psychotic signs and experience psychotic symptoms, and that these are associated with later psychotic illness. In the Dunedin cohort, self-reported psychotic symptoms at age 11 were significantly predictive of schizophreniform disorder (but not affective psychosis) at age 26, though there were many individuals who did not progress to frank psychosis, at least by that age. Those symptoms at 11 were even more strongly associated with earlier neurological signs, delayed language development, lower intelligence and 
emotional development. Violent behaviour in people with schizophrenia was more likely if there had been psychotic symptoms at age 11 [47].

This continuity of illness from tentative beginnings in childhood to frank psychosis in adult life is a constant theme. Welham, Scott and their colleagues [48, 49], using data from the Queensland MUSP birth cohort (n 3,801 assessed aged 5, 14 and 21) recorded self-reported hallucinations in older children (age 14), that represented a significantly increased risk of non-affective psychosis as young adults. They considered that the screening test was useful to identify children at high risk of developing non-affective psychosis.

There has been much less published on affective psychoses, also often assumed to be essentially an adult disorder. In a clinical sample of 529 adult out-patients diagnosed with bipolar disorder between 1996 and 2001, with a mean age of $42,50 \%$ had shown signs of the illness before age 18 . Compared with the other $50 \%$ with adult onset, these had overall greater severity of both depression and mania, more episodes and more days depressed. After 4 years follow up, the group with early onset had a significantly worse record on all measures of illness [50]. This finding that childhood and adolescent onset for bipolar disorder needs replicating from the large cohorts, but, of course, it is a relatively rare condition.

\section{Genetic Influences}

This current review can only briefly outline genetic issues related to psychiatric disorder, but genetics cannot be left unmentioned, and many cohort studies encompass some aspects. There appears to be a consensus that major disorders all have complex multi-factoral aetiologies, and in some cases a genetic component will be a major contributor [51]. A study of 2,111 young adults from a Norwegian Twin Panel examined genetic and environmental factors in many DSMIV disorders [52]. They found moderate correlations for genetic factors apparently distinguishing externalising and internalising disorders, various personality disorders, and eating disorders. They concluded that there were coherent genetic structures underlying these groups, different from environmental correlations, but were aware of the limitations of studying many diagnostic categories together.

For mood disorders, a 2005 review concluded that the genetic contribution in general was very unclear, but evidence suggested a definite influence on bi-polar disorder [25], though the separation from uni-polar disorder in familial associations was not clear [53]. Recent large-scale studies have identified several specific gene loci related to bipolar disorder [54] and the 'Genome-wide association studies'.

In a review of early onset depression in 1996, Birmaher et al., [39] claimed that twin and adoption studies have shown that genetic factors account for at least $50 \%$ of the variance in transmission of child and adolescent mood disorders. Hankin and Abramson [55] say that this is more true for girls than for boys. A recent review of the situation asked why, with improving techniques, and several large-scale gene studies of recurrent early-onset depression had produced only 'promising' findings, though overall 'heritability' has been shown to be quite high [56]. The answer proposed is that unipolar depression has been assumed to be influ- enced little by genetic factors, and therefore has attracted relatively little study, and that what influence there is will almost certainly be multiple genes each having a small effect, often too small easily to be detected. So high heritability is the sum of contributions from several, perhaps many genes, and the grouping may be very varied. However, the authors were optimistic about new techniques for whole genome association studies.

An interesting paper from the Aberdeen cohort of children born 1950 to 1956 showed that children born at full term but weighing less than $5.51 \mathrm{bs}(2,500 \mathrm{gms})$ were more likely to suffer psychological distress (using four items from the GHQ12) in later life, though this was only just statistically significant. Neuro-developmental factors are likely, but what components might be genetic and what intra-uterine experience remains in doubt [57]. Researchers from the Dunedin birth cohort (which has a special emphasis on genetics) have suggested that there is genetic coding which can modify the sensitivity and response styles of children and adults to various stresses and insults, and may, therefore, confer resilience $[58,59]$, and which may work through interference in serotonin transport $[59,60]$. Further work exploring the resilience of some children to serious maltreatment has given some credence to this, though it remains an interesting hypothesis [61]. Paykel considers it now clear from twin studies that genetic pre-disposition to depression also increases the risk of negative life events, perhaps involving partially heritable personality features such as 'neuroticism' [62].

However, genetic contributions have been more favourably considered for schizophrenia and other psychotic disorders. In a critical review of epidemiological evidence, Portin and Alanen [63] considered that twin and adoption studies suggested that susceptibility has, to some extent at least, a basis in genes, but diagnoses are not consistent and there is little specificity. Indeed, when very broad definitions are used, a very high degree of heritability can be obtained, but this diminishes as definitions are narrowed.

Nevertheless, recent researchers in Sweden with the cohort of over one million men born between 1852 and 1982 have confirmed the association of short stature and low BMI with schizophrenia [64]. Using several linked population registers and therefore very large numbers, they have also confirmed clear associations between low birth lengths and later depression where short stature has persisted [65]. More interesting, perhaps, with such large numbers they were able to show a very large excess (OR 4.62; CIs $2.28 \& 9.36$ ) of schizophrenia in subjects whose fathers were over 50 years old at the time of conception, compared with those whose fathers were aged 21 to 24 . They suggest the possibility of de novo germ cell mutations in older fathers having a causal influence.

The large Northern Finland cohort has great potential of exploitation for genetic and family studies and their researchers hope to do this in the future. Isohanni et al., [66, 67] have discussed the issues and summarised current findings from elsewhere: in twin studies, concordance in nonidentical twins has been found to be $10-15 \%$; in identical twins $30-40 \%$ - but that leaves $60 \%$ discordance. Approximately $90 \%$ of people with schizophrenia have parents who 
were not schizophrenic, $83 \%$ have no first degree relative, $63 \%$ no known relative with schizophrenia. The offspring of parents both of whom have schizophrenia are nonschizophrenic in $60-70 \%$ of cases. Researchers are now seeking genetic mechanisms conferring increased susceptibility to environmental factors, but lack of clarity in phenotype definition is a barrier to progress. The pattern is complex, and multiple genes are likely to be involved, expressed through multiple environmental factors, which can also cluster in families. The authors give a salutary message: "Heredity is not a fate, but a kind of probability."

A different approach to studying a rare disorder like schizophrenia is to follow-up high risk groups, the most obvious being children of people with schizophrenia. In the New York High-Risk Project, 15\% of the 79 offspring of schizophrenic parents had schizophrenia by about age 30 (mean of the group, 30.7 years). This will, of course, increase as observations continue, as incidence continues beyond 30, especially in women. But 7\% (four people) of the 57 offspring of parents with affective psychosis also had developed schizophrenia by age 30 . In contrast, there was only one case $(0.8 \%)$ in 133 offspring of parents with no recorded serious mental disorder [68].

Clearly genetic predisposition is important; the situation is complex but has become a lot clearer in the last few years as genetic techniques have improved and interest has widened. Very recently, Nature Genetics gave a whole edition to papers addressing genetic influences on schizophrenia and bipolar disorder. They report several large, indeed huge studies (over 50,000 people) especially in Chinese populations, in which very specific gene loci have been identified associated with schizophrenia [54] and others). The total number of genes or gene variants associated with schizophrenia was at that point 20. Commenting on recent research, Shitij Kapur of the London Institute of Psychiatry said "Mounting evidence from bio-chemistry, brain imaging and now genetics, settles any debate: schizophrenia is at root a neurological disorder. Your genetic inheritance predisposes you to it. It doesn't determine that you will get it, but makes you more vulnerable to it. But thereafter a number of environmental influences, and perhaps social factors, come together to present as schizophrenia." [69].

However, there have been several editorials by highly regarded population genetics researchers, expressing scepticism about whether there are going to be identifiable genes that explain mental disorders like schizophrenia; up to now there has been a general failure to replicate isolated findings. Merikangas, in an illuminating, scholarly article [70] discussed the state of knowledge and the direction of understanding. She saw limiting complexity arising mainly from the continuing inadequacy of our essentially descriptive taxonomy in psychiatry, and the multiple ways in which genes exert any influence they have. In spite of huge advances in genetic knowledge, there seems little early chance of major improvements in either clinical treatment or preventive strategies. Some humility is required; genes need to be seen as risk factors among others, rather than main 'causes', and accurate risk estimations for population action is the challenge.

Kendler [71], in a finely structured argument examines the evidence for genetic contributions to psychiatric illness according to five criteria for simple causal relationship of single gene to 'illness'. Current evidence failed to meet any of the five and he concluded that "The impact of individual genes on risk for psychiatric illness is small, often nonspecific, and embedded in complex causal pathways."

\section{Neurological Deviance in Childhood; Intelligence; Brain Damage or Disorder}

\subsection{Introduction}

It is never easy to measure non-psychotic disorders in populations and relate them to early experiences or features of childhood. Definitions and classifications remain problematic, perhaps especially in their merging with 'normal' experiences of depression, anxiety, fear, guilt, remorse, and so on which are common to all humanity. Psychosis may be a little easier in diagnosis which separates it from everyday experience because of its severity and characteristics, but there are many border-line or indistinct cases. It has been much more studied than others in relation to some childhood determinants.

Yet a fundamental problem for studying psychoses is their relative rarity, demanding huge populations if cohort studies are to provide sufficient numbers of cases for convincing results. Because incidence is progressive over many years, studies also require several generations of follow-up. For example, out of 4,746 subjects followed through to age 43 in the British 1946 birth cohort, only 30 clearly defined cases of schizophrenia were identified, though more, especially among women, would arise later [72]. However, other types of study are also important, including case control studies and followup of high risks such as in the offspring of cases.

The substantial amount of work done on the biochemistry of the brain in relation to neurological deficits described above, cannot be dealt with here, but the large-scale cohort studies focussed upon here do provide some evidence of possible origins of neurological damage, as well as genetically determined disorder. A lot of evidence has now accumulated that signs of neurological abnormality early in and throughout childhood are associated with later schizophrenia and related psychoses, though less for other psychiatric disorders [73]. There is also evidence, not always confirmed, for obstetric and peri-natal problems, and some for later neurological insults such as meningitis / encephalitis, also being associated with serious psychiatric disorder in adults, again especially schizophrenia spectrum disorders.

Low birth weight and short birth length have both been implicated in studies though few have had detailed evidence from birth of obstetric complications, or recorded 'apgar' scores. Delayed early milestones and more obvious signs of neurological deficits have been the focus of many studies, as has measured intelligence. Earlier causes of these indicators may be genetic, pre-natal or both, and the processes at work are usually unknown, but they all imply brain damage or disorder.

\subsection{Schizophrenia Spectrum Disorders}

\subsubsection{Specificity of Risk Factors}

Signs or indicators of neurological deficits, combined with established genetic factors, give rise to most current hypotheses relating to the aetiology of schizophrenia spec- 
trum disorders. There is broad consensus, however, that the causes of schizophrenia and its expression in psychotic episodes are many, inter-related, include genetic factors, very early neurological factors, childhood emotional and psychological factors, and stress factors through the life course [51]. Much of this evidence comes from the long-term birth cohorts [74]. The apparent risk factors are not always specific to narrowly defined schizophrenia [73]. Cannon et al., in 2002 [47] wrote: "the same childhood development risk factors seem to apply to the broader concept of schizophreniform disorder as to the narrower concept of schizophrenia itself."

\subsubsection{Size and Condition at Birth}

Very low birth weight $(<1,500 \mathrm{~g})$ or low birth weight $(<2,500 \mathrm{~g})$ are usually accepted as proxies for a sub-optimal pre-natal milieu, though this might have many causes. Many studies have shown associations with an increased risk of schizophrenia. A meta-analysis in 1995 produced a pooled risk of two times (OR 2.0) for exposure to obstetric complications, but we cannot know if these are themselves the result of pre-existing foetal abnormalities [75]. There was an increased risk in the British 1946 cohort [76], and in the Northern Finland 1966 cohort, where strict definition of severe delivery complications leading to 'peri-natal brain damage' gave an increased risk of x7 (OR 7.0) for schizophrenia. Short gestation (with appropriate weight) was also a risk factor, but not 'small for gestational age'. However, numbers are very small and confidence intervals wide: of 125 survivors of severe peri-natal brain damage, 6 persons $(4.8 \%)$ developed schizophrenia by age 28 . On this basis, about $7 \%$ of all schizophrenia occurring in the population might be attributed to peri-natal brain damage [73].

The Dunedin cohort also found a higher risk of schizophrenia (but note the caution in Section 5.2.1 above) associated with low 'Apgar' scores in the new-born, hypoxia and, in this population, also babies small for gestational age. Other diagnostic groups did not appear to be at risk [47]. The 7,086 births in Helsinki hospitals between 1924 and 1933 had generally good obstetric records. Later schizophrenia was linked not only to low birth weight, but also to small length at birth and low placental weight; period of gestation was not linked. Children with early low body-mass index (BMI) and belowaverage weight at seven, maintaining it throughout childhood, were at higher risk of schizophrenia [77]. There were similar findings in the Swedish conscripts study [78]. This resonates with a late study of the victims of the Dutch Famine during the second world war: children conceived during the height of the starvation in $1945 \mathrm{had}$, as adults, three times the risk of schizophrenia-spectrum disorder, and twice the risk of the most narrowly defined schizophrenia [79]. It is of interest that there was also a notable increase in prevalence of nervous system anomalies in the same cohort in the context of an otherwise stable incidence [80].

Although low birth weight has been associated with schizophrenia in many studies, a record-linkage study on a huge population of men in Sweden revealed an association of schizophrenia not with low birth weight but with short birth length - the shortest babies had the greatest risk. Where that short stature was maintained even in young adulthood and combined with low BMI, there was an increased risk, whereas short babies who became taller were not at increased risk [64, 65].

High birth weight has not been much studied, but appeared to be a risk factor for schizophrenia in males only in the Northern Finland cohort [81] and in the Swedish conscripts study, consistent with a previous Swedish casecontrol study [82] where there was about four times the risk for one standard deviation (SD) above average birth weight [78]. This is interesting in the light of a later meta-analysis of obstetric complications in relation to schizophrenia, which found the strongest predictor of schizophrenia to be diabetes in pregnancy [83]. Since there is evidence of sub- optimal neurological development in infants of mothers with poorly controlled diabetes, there is some face validity in these links [78]. However the much earlier Helsinki (1924-33) cohort did not show any association with high birth weight [77].

\subsubsection{Neuro-Motor Deficits; Infant 'Milestones'}

In reviewing studies of factors below age 4, Kagan and Zentner in 1996 [84] found the most robust association with later schizophrenia to be serious neuro-motor anomalies in infants. Similarly, Ellison et al., in 1998 [85] found a substantial consensus that pre-schizophrenic children were slower reaching their early milestones, had more speech difficulties, more cognitive defects and lower measured intelligence, especially in boys, as well as pre-morbid schizoid traits. A recent review of birth cohort studies came to very similar general conclusions [86]. Where small, high-risk groups have been followed up, studies have shown various delays in development, poor motor function and poor cognitive development [87].

Such neurological imperfections may be indicated by many things in childhood; for example, visual dysfunction at age 4 was found to precede neurological abnormality at age 6 and predict schizophrenia-spectrum disorders in high risk offspring of mothers with a history of psychosis [88]. In the large-scale Child Health and Development Study of over 8,000 people in California, serial measures of growth up to age 9 showed a mean $1 \mathrm{~cm}$ a year slower growth than their peers for women who later developed schizophrenia, but not for men [89].

Early milestones of walking and talking have been found in many individual studies, most importantly in birth cohorts [74]. The British 1946 cohort found late walking, many more speech problems, nail-biting at age 6 , and low educational test scores in those who revealed schizophrenia between ages 16 and 43 (odds ratios - ORs about 2) [51, 76, 87, 90]. Indeed, there was deviance throughout childhood in terms of motor and language deficits [73]. In the Welham et al., review [86], five birth cohorts had studied both early and later motor development and had uniformly found delayed infant milestones and relatively poor motor function continuing throughout childhood, associated with later schizophrenia, whatever measures were used.

However, Jones [72] in 1995 found no evidence of an identifiable high-risk sub-group; rather the evidence pointed to a continuum of risk. The British 1958 cohort showed the same: schizophrenia precursors included motor deficits, many neurological signs at ages 7 and 11 , and lower intelligence test results [73]. An interesting approach was taken by 
Walker and Lewine in 1990 [91] in using 'home videos' from families to look back at the childhood of young adults who became schizophrenic. Late milestones, including standing, walking and potty-training were evident, as well as more clumsiness, poor motor co-ordination, and odd movements.

The Dunedin birth cohort, followed up to age 26, found very similar results to the British 1946 cohort following up to 43. People developing schizophrenia had walked late and had poor motor skills throughout childhood. Expressive language appeared to be normal, but receptive language was impaired throughout childhood, and intelligence was relatively low on tests at ages 3, 5, 7 and 9. These findings were all independent of sex and socio-economic group. They were also associated with self-reported psychotic symptoms at age 11 which were a strong predictor of later schizophrenia [47, $92,93]$. At age 13, schizophreniform disorders were associated with continuing executive and motor impairments, and poor attention but not memory or learning [94]. Lower tested child intelligence was associated with a higher risk of schizophrenia in the Dunedin cohort examined at ages 18 , 21,26 , and 32 [95].

The Northern Finland birth cohort (n 4,976), has found similar relationships: those later developing schizophrenia, narrowly or widely defined, as well as 'other psychoses' were delayed in learning to stand as infants (but not delayed in other milestones), and performed poorly aged 16 at school, especially in motor skills. These associations were independent of any genetic factors imputed by family history [66]. Unusually, in this cohort, pre-schizophrenic pupils did not generally show lower schoolmarks, but, they were far more commonly behind their grade or in a special class. Pupils in a lower class than expected at age 14 because of low IQ scores had an increased risk of schizophrenia, other psychoses, and hospital treated non-psychotic illness, estimated between times two and times eight [96]. At age 31 women, but not men, who had had lower placental weight, lower birth weight, and smaller head circumference at 12 months had a higher risk of 'schizotypal traits' [97]. These authors, from other work, thought the overall results reflected those they got relating to frank schizophrenia.

There are other possible sources of brain damage than during and after birth, and meningitic and encephalopathic infections are the most likely. A small New York study of rubella in pregnancy suggested that exposed infants who became schizophrenic had shown many more neuro-motor and intelligence deficits than those that did not, but such evidence needs confirmation in larger studies [98]. In Sao Paulo, Brazil, a huge epidemic of meningitis in 1971-72 gave occasion to follow-up, though only a small and nonrepresentative sample could be traced and interviewed. Of 190 survivors of meningitis contracted under the age of 4 years, 8 had become schizophrenic as young adults, whereas none were schizophrenic in the 156 sibling controls [99]. Whilst by no means conclusive, this is backed up by evidence of a high risk from meningitis in the Northern Finland cohort who followed children throughout childhood. The adjusted OR for schizophrenia after CNS viral infection was 4.8 'Other psychoses' had an increased risk of 6.9 after bacterial infection, though numbers were small and confidence intervals very wide, in spite of a follow-up population of 11,017 sub- jects $[67,100,101]$. There is some evidence for a relationship with influenza exposure, but not in all studies [80].

\subsubsection{Measured Intelligence and Performance}

Intelligence, however measured is distributed in human populations according a 'normal' or 'Gaussian' curve, being essentially symmetrical around the mean. There is, however, an excess at the lower end related to brain damage and disorder. Apart from that, there are no doubt many causal factors and processes underlying relatively low intelligence, but they are seldom known for individuals, and conventional measurements do not accommodate all types of intelligence and related human capacities. It is, therefore, somewhat controversial to use 'IQ' or other summary indicator as a proxy for neurological deficit, but, accepting that, it has given some value in epidemiological studies. Some justification might be found in large-scale and long-term population cohort studies which demonstrate higher mortality from a wide range of causes at almost all ages related to intelligence measured in childhood, with USA data [102] and with UK data [103].

A meta-analysis in 1984 showed intelligence test deficits related to later schizophrenia, but it would be good to have this replicated 30 years later [104]. The much later, and very large Swedish conscripts study (birth years 1973-1980) showed that 18-year olds with schizophrenic disorders had had lower IQ scores as children, as well as recorded 'inter-personal problems'. Other psychoses showed similar associations but to a lesser degree [73]. In their review of birth cohorts, Welham and Isohanni [86] found that studies addressing the issue "uniformly found that individuals who develop adult schizophrenia (or schizo-affective disorder, or psychotic symptoms), as a group achieved lower scores on intelligence tests in childhood and adolescence than their peers." Birth cohort studies have also usually identified lower performance on verbal, nonverbal and mathematical tests in children who go on to suffer schizophrenia. These may all accumulate problems resulting in poor overall school performance.

On the other hand educational progress has shown rather mixed results, with some showing an excess of later schizophrenia in high performing children.

The cohort of all births in Helsinki from 1951-1960 produced 486 cases of schizophrenia on the national hospital data-base. 400 had complete school records and these were matched with controls. Although there were no significant differences in examination marks, far fewer went up to high school, and generally they did not pass through the school system as expected from their test results. They did have poor co-ordination skills, and more psychological problems for which they were more frequently referred for investigation and treatment $[105,106]$.

The same results arose from the New York High-Risk Project, following up children of parents with schizophrenia and other major psychiatric diagnoses; there were commonly deficits in attention, memory and neuro-motor function at age 15 in those who were becoming schizophrenic [68]. In a meta-analysis of studies with recurrent intelligence tests, Szoke et al., [107] concluded that cognitive function in schizophrenia was essentially stable, suggesting an underlying developmental defect rather than a neuro-degenerative progressive disorder. Murray et al., [108] related adult cog- 
nitive function in schizophrenia to delayed neuro-motor milestones in infancy, suggesting a common over-arching defect affecting all these measures.

\subsection{Affective Psychosis; other Depression and Anxiety}

For affective psychosis and other depression and anxiety there is less evidence, though many studies have found associations to be less than specific within the wider group of psychotic illnesses. In the British 1946 cohort, before serious affective disorder of onset in childhood there were delays in motor and language milestones, speech defects between ages 6 and 15, and lower psycho-motor alertness later between 4 and 11. Some neuro-motor signs, especially twitching and grimacing, persisted into adolescence, especially in boys where the risk was times 2 , but signs were generally less marked than in schizophrenia $[33,73]$.

Low birth weight was shown to be a factor in the cumulative prevalence of depression in 1,420 adolescent girls followed up from age 9 to 16 in N. Carolina, USA, but only interacting with adverse events or experiences. Girls with low birth weight or normal birth weight and with no adversities had no adolescent depression, but each adversity added to the risk in girls with low birth weight far more than the others. There was no such effects in boys [109]. However, their paper does not differentiate birth weights related to gestational age, though they say it was measured.

In a remarkable data set from Sweden, women born in 1914, 1918, 1922 and 1930 have recorded birth factors and psychiatric examinations in 1968,1974, 1980, 1992 and 2000 . Over their varied life-times, $44 \%$ of these 803 women experienced depression, including MDD, 'dysthymia' and 'depression not otherwise specified'. Of birth factors only relatively low birth weight $(<3,500 \mathrm{~g})$ and short gestation were associated with more depression [110]. Exploring possible reasons, Cunnane and Crawford, [111] discuss the importance of adipose tissue - body fat - in the new-born. Similar results were shown by a study focussing upon 'trait anxiety' - "a propensity to experience anxiety intensely and frequently" in over 2,000 men and women of the Finnish cohort born between 1934 and 1944. At a mean age of 61.5 years, higher trait anxiety scores correlated with lower birth weights and BMI, but not length [108, 112].

In the Aberdeen 'Children of the 50s' cohort, they found psychological distress in mid-life (age 45 to 51 years) to be significantly associated with low birth weight $(<5.5 \mathrm{lbs}$; $2,500 \mathrm{~g}$ ) but only when 'small for dates', particularly full term, very small babies. This is a marker of impaired foetal growth and suggests neuro-developmental factors [57].

Affective disorder was also associated with poor coordination throughout childhood, and clumsiness and grimaces at age 15, in the British 1958 cohort. Intelligence and educational tests were relatively poor too, but less so than in schizophrenia [87]. However, the association of low IQ in childhood with psychotic depression and other non-affective psychosis has been supported by later studies [113]. The Dunedin cohort has shown more neurological deficits in childhood associated with major depressive disorder, but only where onset was before the age of 16 [93].
On the other hand, by the age of 32, the Dunedin cohort had shown low IQ to be a significant indicator of both depression and anxiety, persistence of depression and 'comorbidity', when diagnostic assessments were made at ages $18,21,26$, and 32 [95]. Of their suggested explanations, perhaps their most convincing are low IQ as an indicator of neurological deficits conveying increased vulnerability, and low IQ also increasing psycho-social stresses, with lower levels of personal resources to deal with them.

The British birth cohorts have also shown intelligence to be related to depression and anxiety broadly termed 'psychological distress'. They have chosen to emphasise that those with higher measured intelligence as children had a very significantly reduced risk of depression and anxiety at age 30 (1970 cohort) and 33 (1958 cohort) [114]. In a follow-up of the National Collaborative Peri-natal Project, one standard deviation increase in IQ at age seven was associated with a $50 \%$ reduction in cumulative risk of generalised anxiety disorder (GAD) by their mid-thirties [115]. There was a progressive association of more GAD with lower IQ and overall, the mean IQ of those with GAD was 5 points lower than those without, the difference increasing over time.

It is worth noting in this context that studies of Swedish conscripts (over one million men) have recently concluded, inter alia, that lower intelligence is a risk factor for the whole range of mental disorders, for more co-morbidity, and for greater severity of illness [114]. Moran et al., [116] also found in Sweden that lower IQ scores related very positively to later risk of admission to hospital with a diagnosis of personality disorder. More specifically, in the large cohort of 610,359 Norwegians born between 1967 and 1976 and followed up through 2004, the lowest two intelligence groups (considered approximately equivalent to IQ 70-80) had a $40 \%$ increased risk of suicide, even when the analysis adjusted for many other variables including mental illness [117].

Not surprisingly, trauma may affect development. A follow-up of children examined after violence before the age of five, and admitted to hospital with a diagnosis of Mild Traumatic Brain Injury, showed them to have a very high risk of ADHD and conduct disorder, mood disorder and substance abuse at age 14 to 16 , with odds ratios between 3.1 and 6.2 [118]. These behaviours and problems are notable indicators of later mental illness.

\section{Features of Personality}

This heading poses some problems, as 'personality', however measured, is rarely recorded in cohort studies, and where features are recorded, they are couched in terms now not in fashion, considered inappropriate, or representing disputed concepts. 'Neuroticism' might be considered one of these. Other features here reviewed may be considered a mixed bag, but it seemed necessary to group them and, in a general sense, they may be considered to represent features of 'personality', itself not clearly defined!

'Neuroticism' in childhood and adolescence is commonly said to be strongly associated with adult psychiatric disorder but the relationship is not specific, being found for psychiatric symptoms, psychiatric disorder in general, anxiety and 
depression, schizophrenia and related psychotic illnesses. Part of the problem is the personality construct represented by 'neuroticism', not necessarily conceived in the same way by different researchers, as well as the limitations and variations of measurement.

Few cohort studies report on measures of personality. Probably the most standardised measure is the Maudsley Personality Inventory (MPI), used for adolescents and providing, in its simplest analysis, scores for 'neuroticism' and 'extroversion'. This was used in the 1946 British birth cohort at age 16, but at 13 they had used the Pinter Aspects of Personality Inventory [119]. The Christchurch cohort used a short-form version of the 'neuroticism scale' from the Eysenck Personality Inventory at age 14, related to, but different from the MPI, and a 'novelty-seeking scale' from the Tridimensional Personality Inventory at age 16 [120]. The Dunedin cohort used teacher ratings between ages 5 and 9 from the Rutter Child Behaviour Scales for emotional and behavioural functioning, describing 'internalising' and 'externalising' problems [121]. In up-state New York, 10 dimensions of 'temperament' were explored with the 976 mothers in the first interview, the children averaging age 6. A 'severe' problem in one dimension rated the child as having a' difficult temperament' [122].

It is hard to say how results using these different measures might relate to each other, as well as others such as the Bristol Social Adjustment Guide, used at ages 7 and 11 in the British 1958 cohort [123], or, indeed, aspects of behaviour, and psychiatric symptoms measured in children. It is surprising that coping skills and self-esteem are so rarely mentioned in the reports of cohort studies. It seems likely that these too would overlap with 'neuroticism' or other personality measures. Poor coping skills and low self esteem have both been claimed as antecedents of adult major depression, but more evidence would be helpful [39, 124].

Researchers jointly from the Christchurch, Groningen and Canberra cohorts, noting the continuity of symptoms of depression through childhood, adolescence and into adulthood, and the link with 'neuroticism', considered that their studies, at least, provided evidence for an argument that 'neuroticism', as constructed and measured in their cohort research, might be no more than "an account of the subject's stable or typical level of minor psychiatric symptomology." [124].

This may not be far away from the idea expressed by researchers from the British 1946 cohort that 'neuroticism' might represent a general susceptibilty to illness of many sorts, perhaps through lowered stress tolerance [125], and Rodgers' suggestion that 'neuroticism' in girls, as associated with later affective disorder, may represent a specific indicator of vulnerability, perhaps particularly affecting selfesteem, with many knock-on effects on both social behaviour and social experience, such as early marriage and subsequent divorce [126].

Adults in the Dunedin birth cohort rated by teachers and parents as showing 'internalising problems' as children, had significantly more anxiety and depression at age 26 . In the Christchurch birth cohort 'neuroticism' at age 14 predicted major depression in adolescence, with an OR of 2.7 [16].
In the British 1946 birth cohort, following up 3,293 adults at age 36 , and specifically examining the relationship of child personality to adult psychiatric disorder, of seven best predictors of adult disorder (high scores on the Present State Examination - PSE), the best of all was MPI-measured 'neuroticism' at age 16, in both girls and boys, though ORs tended to be only 2-3 [90]. This was true specifically of nonpsychotic affective disorder; there was also a negative association of 'extroversion' as measured by the MPI [119].

They also found that there seemed to be an impact of childhood behaviour and 'neuroticism' upon parenting styles, which have, in turn, an impact upon later depression in their children [127]. They comment: "The offspring of less harmonious families are marked by poorer socio-emotional development which is evident in childhood neuroticism, in aspects of marital, child-bearing and occupational histories in early adulthood, and in several areas of inter-personal relationships in mid-life, all factors held to be important contributors to poor mental health".

A similar circularity of problems was found in New York where 976 families with young children (average age 6) randomly selected in 1975 were followed up for 16-18 years. They found that a young child with a 'difficult temperament' rated on interview with the mother in 1975, was associated with later parent maladjusted behaviour, which was then associated with adolescent and adult anxiety and depression in their offspring [122].

The association of 'neuroticism' with later psychiatric disorder continued to hold true into mid-life for the British 1946 cohort. At age 43, high Psychiatric Symptom Frequency Scale (PSF) scores, and high PSE scores at age 36 were both associated with 'neuroticism' measured at both age 16 and 26. Extroversion was still associated with low scores at 43 [125]. And 1,778 women retained from the 1946 birth cohort, whose health was measured annually from age 47 to 52 showed a progressive significant excess of psychological distress, and consumption of anti-depressant drugs, if they had revealed 'neuroticism' or anti-social behaviour in adolescence [128].

Not surprisingly there has been shown a link with suicidal behaviour. Of 965 children in Christchurch followed through to age $21,29 \%$ had had suicidal thoughts by that age, and $8 \%$ had attempted suicide, both associated significantly with 'neuroticism' and 'novelty-seeking personality', as well as with 'dysfunctional childhood circumstances' [26, 38]. Successful suicide was also linked [120]. Perhaps more surprisingly, the British 1964 cohort found progressively greater excess of natural deaths and accidental deaths, as well as suicides, between ages 16 and 50, associated significantly with earlier 'emotional instability', as measured by the personality inventory [129]. In the Norwegian birth cohort of 1967-1976, suicides were significantly more common in children who were not first-born, especially women, a somewhat surprising result, and unrelated to later mental illness. The authors hypothesise that the birth position affects formation of personality in subtle ways but this is highly speculative [117]. See the meta-analysis by Sullaway [130].

There is an additional dimension to the findings of cohort research relating child personality features with adult psychi- 
atric disorder. Depression is well known to be associated with negative life events, especially threatening events or those involving inter-personal loss. But there are confirmed findings that child 'neuroticism' may increase the risk of experiencing a wide range of negative life events, and this circularity of effect may continue through adult life, possibly by increasing emotional reactivity to minor daily stresses, perceiving them as more serious than others would $[25,62$, $131]$.

In the British 1946 cohort, the association between life events and adult mental health was greater if childhood 'neuroticism' was greater and child mental health was poorer [132]. It has already been noted that the relationship in adolescence between depression and life events seems reciprocal, each modifying the risk for the other in the next phase.Patton et al., in 2003 [40] wondered if features of personality might underlie this phenomenon.

A related concept arising in a different theoretical context, is 'locus of control', considered a trait of personality that represents the extent to which individuals believe they can influence events through their own actions. From the British 1970 birth cohort (original n 17,198), [133] there were 11,261 adults aged 30 agreed to participate, of which 7,551 had full data using the CAROLOC scale at age 10 to measure locus of control. Even after controlling for sex, IQ, educational attainment, earnings and SES, adults at 30 who had shown higher internal locus of control as children had significantly less obesity, overweight, poor or only fair selfrated health, and psychological distress (OR 0.86; CI 0.760.95. Women also had less high blood pressure. Psychological distress was measured on the Malaise Inventory which has been widely used within the British cohort studies. It is possible that locus of control acts through health behaviour: those with higher levels as children showed less smoking, more exercise, and less obesity and overweight as adults.

Something similar but less formalised as a concept was included in the Dutch NEMESIS study which followed up 7,076 adults aged 18-64 for a year, re-interviewing 5,619 for, inter alia, social phobia which were diagnosed in $1 \%$ of the total. There were many associations with childhood phenomena, but prominent among them were low sense of mastery and low self-esteem, together with emotional neglect and poor educational achievement [134].

Several papers recently have looked at positive features of childhood and their impact on adult health. In the British 1946 birth cohort, positive and negative behaviours were assessed at age 13 and 15, and personality was assessed at age 16. Positive childhood behaviour was associated with mid-life well-being: a low probability of life-time emotional problems, high levels of satisfaction with work, a high frequency of contact with friends or family, and engagement in social activities. The associations were independent of childhood SES, cognition and attainment, and adult SES, and recorded extroversion. The interest for this review is that childhood well-being appeared to predict adult well-being, not merely an absence of mental ill-health [135].

Skodol et al., (9) focussed upon personality disorders which are known to tend to remit through adult life. They followed up 520 patients with diagnoses of various personal- ity disorders for four years, re-assessing them six times, and including the Childhood Experiences Questionnaire (Revised). Positive achievements and positive inter-personal relationships during childhood or adolescence were significantly associated with remission from avoidant and schizotypal personality disorders. The more positive experiences and the wider period they covered, the better the prognosis.

It has been found in several studies that neuroticism measured in childhood is associated also with schizophrenia, or schizophreniform disorders in adult life. Twice the expected number of people with schizophrenia were found among those recorded with 'neuroticism' at age 16 in the British 1964 cohort [119], and two to three times for 'neuroticism' at age 14 in the Christchurch cohort [136]. In the British 1958 cohort, at age 11, girls in particular had more commonly been described as 'un-reactive, withdrawn \& unforthcoming' [73].

The Christchurch researchers questioned if 'neuroticism' as measured, confused early pro-dromal signs of psychosis; other reviews have found schizoid traits more commonly in children who later develop schizophrenia [85]. But there is a much wider question possibly relating all these measures of personality with early signs and symptoms of illness, and a wide variety of developmental and neurological signs increasingly associated with a higher risk of schizophrenia in adulthood. This is addressed above in Section 5.

The variables studied in 50,087 Swedish male army conscripts might also be collectively considered to represent a measure of personality. Four variables were associated with an increased risk of developing schizophrenia - having fewer than two close friends; preferring small groups; feeling more sensitive than others; and not having a steady girl-friend. In the presence of all four of these variables, the OR for schizophrenia was 30 [137]. But again, do these really represent early indications or manifestations of the disorder?

Personality disorder research is broad and extensive and overlaps only partly with that devoted to psychiatric illness. This review cannot deal with it thoroughly, but cannot ignore it when it arises in the context of other research.

\section{Behaviour in Childhood and Adolescence}

\subsection{Behaviour, Psychological Problems and Psychiatric Disorder}

Many reviews have reported that children and adolescents with behaviour problems are at an increased risk of psycho-social problems, including depression, in adult life $[16,138-142]$. The possibility of overlap of behaviour with signs of illness and aspects of personality is obvious; behaviour in cohort studies has been measured in many different ways, and may be reported simply as various types of difficult or abnormal behaviour or as a diagnosis of conduct disorder. The main debate on measurement is between dimensional and categorical methods. In cohort studies, the former will often be considered the most useful. There are probably common underlying factors and processes, whatever categories may be super-imposed [143, 144].

There is a similar problem with abnormal or unwanted behaviour in adults: some behaviour may be diagnosed as mental illness; some may be treated as anti-social behaviour, 
or even criminal activity. So outcomes must be studied on a broad front only possible in long-term cohorts. Indeed, a 2009 report from the British 1958 birth cohort of 11,142 children, demonstrated significant long-term excess of overall mortality associated with child problem behaviours, both externalising and internalising, though a greater effect from the former [145]. In the Christchurch 1977 birth cohort of 1,265 children, the extent of conduct problems aged 7 to 9 years was associated with excess problems of recorded crime, substance use and conflictual sexual / partner relationships, as well as mental health, when aged 21 to 25 [143]. Interestingly, they found very similar associations for girls as well as boys, though conduct problems were more common in boys.

Reviews have identified several apparent relationships: impulsivity and lack of control in boys aged 3-4 associated with later anti-social and delinquent behaviour [146]; difficult behaviour in early teenage years, including poor school attendance, associated with adult psycho-social problems [126]; inattention with over-activity associated with later psychiatric disorder [147]; and conduct disorders associated with later depressive symptoms [146]. In a total area sample of 411 South London boys aged 8-10 in 1961-61 followedup to age 32 , adult delinquency and offending was associated (perhaps not surprisingly) with anti-social behaviour as children, but also with impulsivity, hyperactivity and attention deficit disorder [148].

Descriptions and categorisation of child behaviour is most commonly reliant upon parent or teacher reports, which have a very poor rate of agreement [26, 146]. Behaviour observations can only apply to a specific age, for expectations change throughout childhood. Boys and girls must be treated separately. Generally the context of cultural norms is not described in these studies, so comparisons between cultures may not be valid..

Moreover, child behaviour is likely to be related to current family circumstances or recent life events as well as more remote adversities such as parental conflict and divorce. As Fergusson and Horwood wrote [143] "... these disorders are multi-causally determined and reflect the aggregate effects of social, family (including genetic), individual, peer and other factors." To link behaviour to specific experiences is often difficult or impossible, yet the significance of the behaviour is difficult to interpret without doing this. In a 20-year follow-up of London school-children, the only circumstances which appeared to relate to early behaviour problems were severe negative life events [149]. This is made more complex by the knowledge that behaviour itself is a predictor of negative life events, and is probably, in part, a cause of some of them [62].

The overall picture is, therefore, not very satisfactory, and in most situations subjected to research, any associations of behavioural disturbance with later psychiatric disorder are part of a wider picture involving also symptoms and signs of illness, personality features, school achievement records and measures of intelligence, as well as social and family variables. Perhaps for the same reasons the predicted excess of adult disorders is, in most cases, quite small. One review by Kagan and Zentner in 1996 of early childhood factors observed that 'recovery' from early signs of disturbance, as well as adverse family conditions was the norm, unless adversities continued. They offered a positive and optimistic comment rare in this literature: "...children have a natural tendency to grow towards health." [84]

The British 1946 birth cohort found bed-wetting at age 4 and 6 in both boys and girls to be associated with adult psychiatric disorder at age 36 , as was aggressive behaviour at several ages, and anti-social behaviour at 15 in girls, and frequent truanting at 15 in boys [90]. For women recorded annually between ages 47 and 52, psychological distress was significantly greater if anti-social behaviour had been recorded in adolescence, often linked with neuroticism [128]. And, more surprisingly perhaps, bed-wetting, and conduct disorder, often linked with emotional instability, were associated with significantly greater mortality - natural, accidental and suicidal - with increasing risks between ages 16 and 50 [129].

In the British 1958 cohort, deviant behaviour at 16 was associated with depression between 16 and 23, and disruptive behaviour with early motherhood in girls, itself linked with later depression. Examination of both the 1958 and 1970 cohorts suggested that the association of disadvantageous circumstances in early childhood with poor psychological health in adulthood involved maladjusted behaviour in intervening years [150]. As with aspects of personality, there may be circular effects: a 20-year follow-up of London school-children showed that high scores at age 10 for behaviour problems predicted later negative, even severe threatening life events in both men and women, but stronger in women [149]. This suggests at least close links between measures of personality and measures of behaviour in children.

In Christchurch, anxiety and depression at ages 16-18, and 18-21 were predicted by withdrawn behaviour at age 8 , but this was often also concurrent with anxiety. In Dunedin, conduct disorders were associated with later schizophrenia, and attention deficit and physical aggression were risk factors for violence if schizophrenia developed [47, 151, 152]. Aggressive boys who did not graduate to adolescent delinquency to any degree, were at higher risk of anxiety and depression by age 26 , were socially isolated and tended to have finance and work problems [153]. Among 6,000 Finnish army recruits, previous conduct disorder predicted psychiatric disorder at age 18, but in a very non-specific way, that is, including anxiety and depression, psychosis, substance abuse and anti-social personality disorder. Thus there may be a very high risk of adult psychiatric hospital admission in children with problems of inattention especially when concurrent with conduct disorders [154].

It might be noticed here that there is good evidence of childhood behavioural antecedents to schizophrenia, but they are not easily identified as such. Welham et al., [86] undertook a review of the evidence and concluded: " The behavioural antecedents of schizophrenia are subtle. Individuals who later develop schizophrenia are not marked by extreme deviations in behaviours. Also most cohort members with a behavioural feature associated with later schizophrenia, do not develop the disorder. In other words, behavioural features have poor positive predictive value." 
The associations may be even wider: in a rather small follow-up study of children aged 5-12 in San Francisco, USA, conduct problems, hyperactive-impulsive behaviour and inattention problems were strong predictors of criminal behaviour 18 years later [155]. In a high crime community in Seattle, self-reported conduct problems at age 10 was the only predictor of depression at 21 [156]; in a very poor community in Chicago, teacher-rated aggressiveness in girls predicted major depressive disorder at age 32-34 [157].

As expected, behaviour problems in children are themselves predicted by many family and social factors in earlier childhood [158]. And early behavioural problems tend to be associated with later behaviour problems [159], sometimes linked also to depression [23]. Recent intervention studies have brought a degree of optimism for preventive programmes, but resources are not commonly available.

\subsection{Cannabis Use}

A particular aspect of behaviour which has been the subject of several studies is the use of cannabis. In both the New Zealand cohorts, Christchurch and Dunedin, cannabis use was predictive of schizophrenia before there were any signs of psychosis [160]. In Dunedin, the earlier the use, the greater the effect; users by age 15 had four times the risk of diagnosis of schizophrenia by age 16 than non-users, but most had shown some symptoms at age 11. Cannabis use was also associated with more violent behaviour as young adults, but was not associated with more depression. Interestingly, use of other drugs did not predict schizophrenia when cannabis use was taken into account [160]. In a review in 2005, Isohanni et al., [67] quote evidence of higher rates of schizophrenia and psychotic symptoms related to earlier cannabis use in Northern Finland, 50,000 Swedish army conscripts and in other studies.

Over two thirds of the Christchurch cohort had used cannabis by the age of 21 , and $21 \%$ were assessed as abusing or dependent. More than a quarter had used other illicit drugs and $7 \%$ were assessed as abusing or dependent. Cannabis use preceded use of other drugs in $99 \%$ of children, and those who used it 50 or more times a year were 140 times more likely than non-users to use other illicit drugs later -60 times when adjusted for all possible variables. Researchers concluded that almost certainly cannabis leads to other drug use [121].

Two reviews, both published in 2004, offered different views of the literature on cannabis and psychosis. Arseneault et al., (ibid) focussed mainly upon five prospective analyses from four longitudinal studies in Sweden, The Netherlands and New Zealand as giving the best evidence and permitting pooling of results [51]. They found a pooled odds ratio (OR) of 2.34 (CI 1.69-2.95) and concluded that cannabis use is not a sufficient or necessary cause but is a contributory cause, roughly doubling the risk of schizophrenia. Most users will not become psychotic, but a vulnerable minority will, and this probably includes the youngest users.

However, Macleod et al., in 2004 [161] used a wider range of study types, and they viewed the overall results with profound scepticism, focussing on the many technical possibilities which would cast doubt on results associating cannabis use causally with schizophrenia. However, they do say that "Cannabis use might exacerbate existing predispositions to psychological problems", and that younger users are at greater risk, which is, perhaps, not far from the conclusions of Arseneault et al.

The Queensland study of Hayatbakhsh et al., [162] also provides a possible corrective. In a birth cohort study examined at age 21 (n 2,493) and using the CIDI-Auto, 51.4\% reported ever having used cannabis, and $21.1 \%$ had had a 'cannabis disorder'. But gender, marital disruption of parents, maternal smoking, child school performance, childhood sexual abuse, early smoking and alcohol consumption, and adolescent aggression or delinquency were all strongly associated with both cannabis use and cannabis disorder, which were even more strongly associated with multiple risk factors. So the context of cannabis use must be understood if any longer-term consequences are to be elucidated clearly.

\subsection{Bullying}

Although it is generally acknowledged that bullying is well known in most schools and in most countries, the frequency of bullying is not easy to define and record, even less to compare. In a study of 25 countries covering 113,000 students aged 11 to 15 years, involvement in bullying (being bullied or aggressive to others) varied from $5 \%$ to $54 \%$ [163]. In a sample of 2464 Norwegian adolescents 5\% reported having been aggressive to others 'often' in the previous 6 months, boys more than girls. This was associated with parental divorce, low self-esteem, depressive and other symptoms, and delinquency [164]. Sourander et al., [165] had similarly found that bullying and being bullied were both associated with poorer family functioning, parental violence and parental mal-treatment. Lien et al., [166] found strong evidence that bullying among Norwegian school children diminished sharply after age 15/16.

There is a substantial literature on bullying from educational and psychological perspectives which has focussed mainly upon effects in school-age children and adolescents (but see Section 9 below). Those driving this research have concentrated upon practical programmes in schools to prevent bullying and there are now several very encouraging evaluation studies of such programmes in several countries. This literature cannot be fully reviewed here but summaries and further references can be found in Olweus and Limber and associated texts [167-170].

Only recently has the wider psychiatric epidemiological literature reflected the much more widespread general interest in bullying. Studies tend to focus far more on victims than perpetrators, (for which see Section 9.2 below) but there is an overlap with some children falling into both categories eg [170]. In the Finnish 1981 cohort of 5,038 children born in 1981, girls very rarely bullied, but boys that bullied $(6.0 \%$ of all boys), and those who both bullied and were victims of bullying $(2,8 \%)$, were more likely than non-bullies to be using anti-depressant or anxiolytic drugs at age 24 [165]. However, the excess could be fully explained by their own psychological problems at age 8 . The most disturbed young men were those who had been both bullies and victims. Almost half had experienced either psychiatric hospital or drug treatment by the age of 24 and almost all of them had shown distinct signs at age 8. Duke et al., [171], in a very large 
study of older American school children, found substantially more (x 2-3) bullying (and other violence) by children with an experience of abuse or family disfunction. Cultural differences may prejudice application in other communities.

Linking bullying behaviour to later psychiatric disorder or suicidal behaviour really needs longitudinal studies. Klomek et al., [172], in a recent review of 300 studies identified of all types, found only 31 articles useful. From crosssectional studies there were suggestive associations of suicidal ideation or attempts with previous bullying behaviour, including the very new activity of cyber-bullying, but methodological variation and inadequacy made interpreting correlations hazardous. The very few longitudinal studies including their own [173], suggested that boys and girls are very different. Only boys showed correlations of bullying behaviour with later suicidal behaviour, with ORs from 1.7 to 11.8 , but this was not sustained when existing depression or conduct disorder was introduced into the analysis. Few girls were found to bully so no comparisons could be made. This is work in progress, but it is fair to see children who bully at school as themselves vulnerable and needing help.

In this context it is interesting to reflect on some findings of large-scale surveys of child abuse which include information on the perpetrators. Radford et al., [174], in a national study in the UK with a representative sample of several thousand, found that of $0.5 \%$ of children under age 11 , and $4.8 \%$ of children aged 11-17 who reported contact sexual abuse, $65.9 \%$ of perpetrators were other children under age 18. There are important psycho-social and cultural issues here, and perhaps this should be considered a special and extreme form of bullying, but studies of perpetrators themselves are few.

The work of Olweus and others referred to above suggests that preventive programmes in schools can work, through measures to change the whole culture of schools, involving all teachers and all children, parents, and even sometimes the wider community. They should be more widespread [175, 168].

\section{Poor School Performance; Poor Educational Achievement}

School is a universal requirement in western societies and has clear end-points which include some measures of achievement. These offer relatively secure and comprehensive data, which also generally ante-date the onset of most serious psychiatric disorder. School performance can be measured as years of schooling completed, qualifications achieved, examination marks, or class grades related to measured intelligence or previous expectations.

Difficult behaviour of any type in childhood is likely to have an impact on school performance and achievements, and it may be these that, in part, mediate the higher risk of depression and anxiety. Inadequate education measured either in qualifications or years of full-time study is one of the key precursors of adult depression and anxiety related to social inequalities; measures of 'size of effect' give ORs between 1.5 and 3.0 [176].

Many important studies have found poor school performance to be related to adult psychiatric disorder. The British
1946 birth cohort found poor educational achievement related to overall psychiatric disorder by age 36 (measured on the PSE) and 43 (measured on the PSF) [73]. Schizophrenia was specifically and progressively associated with low school test scores at ages 8,11, and 15, which also predicted adult affective disorder (ORs around 1.5; somewhat higher, if onset of depression was in childhood, but $<2.0$,) with or without a history of childhood affective symptoms [33, 76]. The British 1958 cohort also showed poor educational achievement associated with psychological distress measured on the Malaise Inventory between the ages of 16 and 23 [177]. Jointly analysing both cohorts showed disruptive behaviour associated strongly with poor school performance and few qualifications, which themselves predicted a wide variety of psychological and social problems in adulthood [178].

In a Finnish cohort study reported by Lehtinen et al., [179], depressive symptoms were not clearly and systematically predicted by measures of school performance, though some groups showed some associations. Their conclusion was that the relationship between relative failure at school and later depressive illness was not a simple one. However, in the massive cohort of 610,359 Norwegians born between 1967 and 1976, suicide (which has some important associations with depressive illness and symptoms) showed a strong inverse association with educational level in both sexes, that is, the lower the educational level, the higher the suicide risk $[117,168,175]$.

For the Northern Finland 1966 birth cohort, data were available for exam marks and appropriateness of grade or class at age 16, as well as diagnoses from the national healthcare registers up to the end of 1994. All three diagnostic groups - schizophrenia, other psychoses, and non-psychotic disorders, were between two and eight times more likely to have been in a class or grade lower than expected (according to known measured intelligence or earlier school performance), or in a special class, than subjects with no psychiatric diagnosis by age 28 (non-psychotic disorders OR 2.2 for men and 4.9 for women; higher ORs for psychosis). Adolescents later hospitalised for non-psychotic disorders had significantly low mean examination marks, but, in spite of also being in lower than expected grades, this was not so for the groups with psychosis [96].

Similarly, in the Helsinki cohort born 1951-1960, children who were later diagnosed with schizophrenia did not necessarily have low marks compared to other children, but tended not to have proceeded as expected within the school system [105]. The implication is that intelligence and academic performance were not factors, but rather features of personality, inter-personal skills, social performance, or other aspects of behaviour. The Finnish study of national service recruits at 18 also showed psychiatric disorder at 18 related, inter alia, to poor school achievement (about twice the risk for affective disorders, but no excess for anxiety) [42]. The same thing has been found in the USA, where poor school performance was one factor related to anxiety and depression in young adults [180].

MacCabe et al., [181] followed the huge Swedish national cohort born between 1973 and 1983 (n 907,011), and 
found poor school performance at age 16 associated with an increased risk of adult schizophrenia (HR 3.9; CI 2.8-5.3), schizo-affective psychosis (HR 4.2; CI 1.9-9.1), and other psychoses (HR 3.0; CI 2.3-4.0). Interestingly, receiving an 'E' grade in any subject at school significantly increased the risk of later schizophrenia, and no birth or social variables confounded these results.

A different approach was adopted by Almquist [182] in his study relating peer-status to later disease specific morbidity in adulthood. He used data from the Stockholm birth cohort of 1953, in which the social status of each child was socio-metrically assessed by his or her peers when they were age13 or 14. Hazard ratios related to adult illness were calculated from in-patient care between 1973 and 2003. Some of the highest HRs were for psychiatric illness associated with behavioural disorders (including alcohol abuse and suicide behaviour), which were not explained by family socioeconomic status. Though little studied, peer status might be an important indicator, but it needs un-picking, as it might reflect many personal, societal and school features. How much it reflects school academic performance per se, is difficult to say.

\section{Childhood Adversity; Life Events; Multiple Disadvan- tage}

\subsection{Adversities and Adult Mental Health}

Definitions of childhood adversity are truly problematic; they inevitably overlap with apparently more specific variables such as negative life events, child abuse or family conflict, and must be expected to inter-relate with variables such as divorce of parents, child behaviour and child psychological disturbance. Not surprisingly, 'measures' cover a wide range of observations, often retrospective, regarding family poverty, poor material circumstances, inadequate housing, and instability of residence, amongst other aspects of environment and experience. It is not always clear from published reports at what periods of childhood these circumstances have prevailed, or for how long.

Hardt and Rutter [183] reviewed studies using retrospective reports on childhood adverse circumstances, and concluded that they will include many false negatives and substantial measurement error, but few false positives. For example, several American studies of women with proven child abuse 20 (or so) years earlier, interviewed with no overt connection with the previous abuse experience, gave a history of abuse in less than two thirds of cases. The greater the detail, the less the agreement. Low agreements have been consistently found in reports from two parents or from parents and their children. However, some studies comparing prospective observations and retrospective recall, have found more false positives than false negatives. Of two recall studies of close sister pairs, one reported better than chance agreement of abuse as children, but in the other the only agreement was when both claimed to recall the same adversity, that is, experienced by both of them; if not there was virtually no agreement.

Hardt and Rutter judged that effects are more likely to be under-estimated than over-estimated, and that retrospective recall methods are only suitable for clearly defined major adversities, not for details, or where much judgement and interpretation is required However, it is intrinsically far more difficult to test false positives than false negatives. Where false negatives can be demonstrated, there must, by definition, be data available relating to the period being recalled, but for false positives there will rarely be data from any source to verify claims. And, of course, there may be many of either not verified. This will be especially true for general population samples, but cohorts remain the best hope of achieving estimates of validity.

More recently, Hardt et al., [184] have tried to throw clearer light on this issue with a comparison of data from the prospective British 1958 cohort (n 7710) and the retrospective Mainz Adverse Childhood Experiences Study $($ n 1,062) in Germany. They examined ten adverse childhood experiences considered to have good evidence for serious consequences, and psychological adjustment at age 42 . No significant differences in risk effects were detected, using bivariate analyses on all ten adversities and a multi-variate model. Retrospective ascertainment appeared to offer no disadvantage.

Comparing the results of different studies from different populations is obviously problematic, compounded by the finding in some studies that a category 'multiple adversities' is the one most clearly related to later psychiatric disorder $[26,51,185-187]$. Some elements may be mutable but it is not easy to know which individual elements are most important in particular populations.

For example, from the British 1946 birth cohort, Rodgers wrote [188] that early environment showed very little relationship with adult psychiatric disorder as measured on the PSE (which records only symptoms experienced in the previous month, and is likely seriously to under-estimate associations), but that high rates of adult illness were related to multiple disadvantages in childhood. High risks of adult mental illness were in relatively small groups who had had particularly bad experiences in childhood, that is, they scored the most in the category 'multiple adversity'. The elements constituting 'adversity' were very limited; for example, no record of child abuse or neglect was included, nor parenting quality and style, and in this immediate post-war cohort parental divorce and separation rates were very low compared with later cohorts. Nevertheless, the highest level of 'multiple adversity' gave increased risks, compared with the lowest level, of over five times in men and over seven times in women.

More recently this cohort has shown early adverse circumstances to be associated with diminished cognitive ability (verbal ability, memory, speed and concentration) not only in childhood and adolescence, but even to the age of 53, and related to poor school achievement and low adult social class [186]. Diminished cognitive ability and poor school achievement are independently associated with higher risks of later mental illness.

The Christchurch birth cohort study has contemporaneously recorded a wide range of adversities including child abuse and impaired parenting. As in Rodgers' analysis of the 1946 British Birth Cohort, Fergusson and Horwood [26] found in Christchurch NZ, that though most individual ad- 
versities gave only modest associations with later mental illness, children exposed to one were frequently exposed to several. They therefore analysed adversities collectively as degrees of 'multiple disadvantage', and found that the $5 \%$ of children exposed to the greatest childhood disadvantage which included family dysfunction, had 100 times the risk of adolescent psychiatric disorder compared to the $50 \%$ most advantaged children. This dramatic result is hard to ignore!

Young people reporting four adversities were over 3 times more likely to have suicide ideation and almost 5 times more likely to have attempted suicide than those reporting no stressful life events in the previous year. Their overall measure of multiple adversities combining many factors, was also progressively related to suicidal ideation and suicide attempts. In the whole cohort of 965 children followed up with full records to age $21,28.8 \%$ had entertained suicidal thoughts, but the $10 \%$ with the highest level of multiple adversities had more than 7 times the rate of the $50 \%$ with the lowest level of multiple adversities. $7.5 \%$ of the cohort had attempted suicide by 21 , but the $10 \%$ with the highest level of multiple adversities had over 60 times the rate of the $50 \%$ with the lowest (31.1\% v $0.5 \%)$ [16].

These findings are consistent with the results of a wideranging review of risk factors for suicidal behaviour in adolescents and young adults by Beautrais [37], again related to a wide range of adversities, usually multiple: "The account that emerges suggests that suicidal behaviours in young people are frequently the end point of adverse life sequences in which multiple risk factors combine to encourage the development of suicidal behaviours." These adverse experiences included social disadvantage, parental separation or divorce, parental discord, impaired parent-child relationships, parental psycho-pathology, and physical or sexual abuse.

The same thing has been shown since then in many studies; though individual details may vary considerably, the overall conclusion is very often that the accumulation of a variety of adversities or disadvantages is the most prominent indicator of later depression and anxiety or broader psychiatric disorder, usually excluding psychosis. They also commonly find that there is a sort of group dose-response relationship with psychiatric outcomes, where the more individual adverse childhood experiences (ACEs), the greater the problems later on. Some recent studies have used a standardised ACE score with the same result whether the outcomes were as specific as the use of prescription drugs, or as broad as a range of psychiatric disorders [189, 190].

Perhaps some light on this is thrown on this by a large longitudinal community study (n 7,485 aged 20-64) in Canberra, Australia, focussing upon the precursors of adult personality traits [191]. Higher levels of cumulative childhood adversities (five or more) substantially increased the risk of high levels of neuroticism (OR 2.6) or negative affect (OR 2.6), behavioural inhibition (OR 1.7) and dis-social behaviour (OR 1.7). Maternal depression also had a substantial significant effect on neuroticism and negative affect. These, of course, can underlie mental ill-health, especially depression and anxiety. It is also noteworthy that Stansfeld et al., [192] in the large Whitehall study found that childhood adversities and parental depression increased the risk of insecure adult attachment styles (features of personality related to capacity for effective relationships) which are also associated with depressive symptoms.

Similar conclusions arise from three important papers deriving from the American National Co-morbidity Survey Replication of 2000-2001, encompassing a group of 12 known adversities. They were all highly prevalent and intercorrelated, and collectively were positively and significantly associated with all outcomes. There was a little nuancing of the large group of adversities: those indicative of maladaptive family functioning (parental mental illness, substance abuse disorder, and criminality; family violence; physical abuse; sexual abuse; and neglect) were more consistently correlated to to impaired function related to psychiatric disorder and the time of onset, which persisted throughout life as far as yet studied. Little specificity was found, and adversities were often clustered; but the more adversities could be counted, the worse the outcomes [39, 55, 193-195].

Benjet et al., [196] had similar results from the Mexican NCS: all adversities (except economic adversity and parental death) were significant predictors of adult psycho-pathology; those representing family dysfunction were the strongest; and the greater the number of adversities, the worse the outcomes. Pirkola et al., [197] in Finland with a sample of over 4,000 people aged 30-64, found "a moderate dose-response relationship between the total number of childhood adversities and current disorders." But looking at the detail, he also found some marked gender differences representing complex interactions of a very diverse set of adverse circumstance and experiences.

Differences between girls and boys are not consistent in the cohort studies available. In the Dunedin cohort a rather limited cumulative measure of childhood family disadvantage (including parental separations, low SES, poor family social support, and poor maternal mental health) showed multiple disadvantage to be associated with psychiatric disorder (DSM III R) in males at age 18, but not in females [41]. In Woodlawn, one of the poorest districts (99\% African-American) of Chicago, USA, where a cohort of 1242 children were followed up from first grade at school to age 32 , poverty predicted adult major depression only in boys [198], but single parent and absence of mother predicted suicidal behaviour in both sexes [198]. There was no multiple or summary measure analysed.

In the Netherlands NEMESIS study, the largest odds ratios for suicidal behaviour were for abuse variables and the smallest for the parent bonding variables, but, even so, "increasing numbers of adversities were associated with progressively higher ORs for suicidal ideation and attempts." [199] Even in China, a similar study in Beijing and Shanghai produced similar results. Adversities were highly intercorrelated; a family dysfunction cluster gave the strongest association with psychiatric disorder outcomes; there was little evidence of specificity and the total number of adversities was important [189, 190, 200].

A welcome variant on this theme comes from Norway [201]. A huge cohort of all males born in Norway in 19671971 (n 170,678) was followed up for 4-9 years from age 29 using national registers. Social inequalities were strong and consistently graded for all psychiatric outcomes. Of many 
variables derived from children and their parents subjected to complex analyses, the best indicator of social position was education level achieved by age 29 . But, the many variables representing characteristics of children and parents that included adversities of childhood, moderated the social gradient of psychiatric disorders in adult life, though not equally. Early childhood factors were more strongly related to 'all psychiatric disability', 'neurosis', 'personality disability' and 'sickness absence', than with suicide and schizophrenia disability rates. They concluded that early factors account for a large part of the socio-economic class inequalities in psychiatric disorder.

The same issue was addressed by Melchior et al., [202] using data from the Dunedin cohort of over 1,000 children born in 1972-3, followed up to age 32. Childhood socioeconomic status (SES) was measured at multiple points between birth and 15 years. Many individual, family and social adversities / risk factors were recorded, and health, including psychiatric disorders evaluated at age 32. Low childhood SES was associated especially with a significantly increased risk of substance dependence and poor physical health in adulthood, but on further analysis, the risk factors included collectively accounted for $55-67 \%$ of poor health outcomes associated with low SES.

In the British 1958 birth cohort, socio-economic adversity, using indicators such as housing tenure and parental financial difficulties, was a definite risk factor for adult anxiety and depression (on the MI) only in women [203, 204]. Power et al., concluded that anxiety and depression is largely of social causation but including many factors that go back to early childhood [187]. Virtually all factors examined gave ORs of approximately $2.0-3.0$. Childhood adversities may also be linked to early pregnancy and behaviour problems in childhood and adolescence which themselves are associated with adult anxiety and depression $[150,205]$.

In an interesting and unusual study, 1,112 children in a very poor district of Copenhagen, identified in 1948-1952 as being under the age of 7 and having been admitted to hospital within the previous five years $(71 \%$ of all children under 7 in the district), were re-identified through the Danish national registers and followed-up after about 30 years [206]. The 430 children experiencing the worst combination of social conditions in the first 7 years were compared with random controls born in Copenhagen in the same period (but for whom the early conditions were not known).

For all diagnostic groups the index group had more hospital admissions in all periods compared with the controls. Psychiatric disorders diagnosed in somatic hospitals, psychiatric disorder following other disease, alcohol dependence, schizophrenia, other psychoses, and suicide, all showed approximately three times the admission rates. There were also large differences for drug dependence, personality disorder, injuries, poisonings, and neoplasms, as well as criminal convictions, unemployment and disability before the age of 40 . Psychiatric admissions in total were just over twice those of controls.

The authors conclude that, in spite of a 'comprehensive' welfare state throughout their lives, early adversities seem to have determined continuing serious disadvantage across most aspects of life. The two most powerful predictors were quality and density of housing, but these must be closely related to many other adverse conditions, and, perhaps are as much symbolic as directly causal. Nevertheless the results of this study are highly salutary. It would be good to replicate this study in later cohorts and different cultures, but few populations could offer the combination of records in early childhood and comprehensive registers to identify them later.

An interesting side light is thrown on this Copenhagen study by an 8 year follow-up of a group of 9-13 year old Native American children raised initially in poverty but with a dramatic change in material circumstances unrelated to family effort or behaviour, welfare provision, or the involvement of any authorities or professionals [207]. The key event was the establishment of a casino in the American $\mathrm{Na}$ tive Reserve, which brought great wealth to the whole community and all families within it. There was a reduction in conduct disorders among the children, but no subsequent reduction in anxiety or depression. It seems that the effects of early adversity may not be so easily reversed by removal of poverty alone; primary prevention of serious adversity in young children may be necessary if we are to diminish later anxiety and depression.

Distribution of schizophrenia and other psychoses according to socio-economic status has long been controversial, and is no less so now as genetic evidence increases. Wicks et al., [208] applied five different indicators of SES to the 2.1 million persons born in Sweden between 1963 and 1983, and looked for hospital admissions for psychotic disorders between 1987 and 2002. Increased age- and sexadjusted hazard ratios for schizophrenia and other psychoses were found for all childhood socio-economic indicators, and ratios increased with the number of adverse social factors recorded. They consider that 'social drift' as an explanation of SES inequalities is unlikely in only two generations. This again suggests that there is a far from simple interaction between genetic and familial determinants and other factors in childhood.

Definitions of poverty tend to be both relative and variable within and between different societies, but researchers must generally use those current in their community. Exposure to poverty in Brisbane, Australia was examined in a birth cohort of 2,609 children and mothers and followed up at ages 14 and 21 (45). Family poverty was found to predict higher rates of adolescent and young adult anxiety and depression, greater frequency of child exposure to poverty resulting in ever more morbidity. In a Brazil birth cohort from 1982 with over 4,000 subjects, greater poverty either as a child or at adult follow-up was related to greater risk of common mental disorders (largely anxiety and depression) [209].

More specifically, Vinnerljung et al., [210] examined psychiatric morbidity among former child welfare / protection clients and controls within a Swedish cohort population of almost one million. Former child welfare clients (risk ratios standardised for year of birth and sex) were four to five times more likely than peers in the general population to have been hospitalised for suicide attempts. They were five 
to eight times more likely to have been hospitalised for serious psychiatric disorders in their teens, four to six times in young adulthood. There were also higher risks for psychoses and depression. Individuals who had been in long-term foster care tended to have the most dismal outcome. Adjusting for birth parents' hospitalisations with a psychiatric diagnosis or substance abuse, and for birth-home-related socio-economic factors, reduced excess risks to around two-fold.

In recent analyses from the British 1958 birth cohort, 'manual' socio-economic position (the lowest SES) at 42 years of age was associated with a significantly increased risk at age 45 of a category 'any diagnosis' and with depressive episode, but not generalised anxiety disorder, in women but not in men. The effects of childhood socio-economic position were deemed to be largely mediated through adult socio-economic position, through pathways that lead from disadvantaged childhood to disadvantaged adulthood [211]. Many things were associated with childhood SES at age 45, including and especially depressive symptoms and chronic widespread pain with a general trend of deteriorating health from social class I to V. Child SES and adult SES at age 45 tended to act as independent variables [212].

More specifically, the relationship between childhood adversities and adult psychiatric disorder could be affected by other experiences in adult life. In papers considered largely in Section 10 there is sometimes mention of the continuing or re-victimisation into adult life of some child victims of sexual abuse, but not much discussion [213-216]. Clearly this must have some bearing on the relationship with disorder. But other adult negative life events have not often been examined in the context of the correlation between childhood adversities and adult illness. Korkeila et al., [217] were able to do this for depressive illness with a large shortterm cohort of 16,877 adults from a random health survey of the Finnish working-age population. They were as meticulous a possible through postal questionnaires in measuring childhood adversities and later life events, and had three records of depression - The Beck Inventory by post, and hospital records of use of anti-depressant drugs, and hospital admissions.

They found the expected correlations between childhood adversities and adult depression (as recorded in all three ways), and between negative life events and depression, but also an increased risk of negative adult life events, especially of more burdonsome events, with childhood adversities. Such life events in adulthood appeared to mediate about one quarter of the effect of adversities upon illness. Their interesting conclusion noted that childhood adversities may influence later depression through production of stressors (negative life events) rather than through sensitization to stressors, or that for some of those with depression, childhood adversities and adult life events represent ongoing exposure to chronic sources of stress.

\subsection{Life Events Including Physical Illness}

We can now consider life events more generally. There is a large psychiatric literature on life events experienced by adults and related to psychiatric disorder, but not very much on children's experience of life events. Several recent reviews and papers note some of the childhood antecedents of adult life events, but not life events occurring in childhood $[62,132,218-220]$. They are mentioned incidentally in Colman and Jones 2004 review [51] of birth cohort studies, and as a considered factor in Beautrais 2000 review [37] of risk factors for suicide in young people. However, a short followup study of about 2,000 adolescents in Victoria Australia, found life events predicting depression between the ages of 14 and 17 , but found a reciprocal relationship, each modifying the risk of the other for the immediate future [40]. In other words, negative life events may increase the risk of depression, but depression may also increase the risk of negative life events. On the other hand, early adversities and negative life events were associated with anxiety in adolescence but not depression [221].

Physical illness in childhood can be considered one of many possible adversities, and some studies have found it associated with later depression [222]. If the British 1946 and 1958 birth cohorts are representative, about $10 \%$ of children experience a physical illness of more than three months duration. However, the results in terms of later psychiatric disorder were mixed: in the 1946 cohort at age 36, women with psychiatric disorder had had significantly more illness in childhood, but at 26 there had been no difference. Perhaps the clearest result was that the highest incidence of psychological problems was in the group with serious childhood disabilities [177].

There was significantly more depression in those who had suffered chronic ill health in childhood in the 1958 cohort, but the differences were very small [223, 224]. Repeated headache in children also had a small association with later psychological symptoms [225]. In the British 1970 birth cohort there was an interesting association between high scores on the Malaise Inventory and self-reported 'chronic fatigue syndrome' (CFS) / 'myalgic encephalomyelitis' (ME), (somewhat ambiguous diagnostic terms related to persistent fatigue without pathology or explanation) both reported at age 30 . They were both associated also with having experienced a limiting long-standing medical condition in childhood [226]. Predominantly sedentary behaviour in childhood increased the risk of $\mathrm{CFS} / \mathrm{ME}$; low rates of both $\mathrm{CFS} / \mathrm{ME}$ and malaise were found in those who had had high levels of physical activity in childhood and adolescence. This was not related to school programmes; that is, it was independent of school sports cultures and opportunities.

At the opposite end of the scale of follow-up studies from the Copenhagen one described above, it is not often that a single clearly defined event or experience common to many children can be rigorously studied, but the removal of many new-born children from their parents to protect them against tuberculosis in Finland between 1945 and 1965 provided an opportunity [227]. 3,020 babies were removed into special nurseries for an average of 7 months, and were followed up in 1998 by means of the national hospital discharge register together with two matched controls for each case. Treatment in hospital for depression (implying serious illness) showed a small significant excess in the separated children compared with controls for men, but no difference for women. Psychotic depression showed no difference. Overall, by this measure, the effect of the separation was very little if anything. 
As already said, bullying is well known in schools and consideration of bullies is in Section 7 above. Here it is appropriate to consider the possible effects of being bullied. At the extreme end, bullying might be considered as child abuse or child trauma where peers are the perpetrators, but mostly it seems to fit best with adversity / negative life events, even where it is repeated over a long period. Like child abuse, it is common, though an international comparative study found huge variation in reported frequency from $5 \%$ to $54 \%$, not clearly explained $[163,228]$. There is a standardised measure available in the 'Olweus Bully/Victim Questionnaire' [229].

Although the focus of this review is adult consequences of childhood experience, children have immediate responses of course. There have been many studies showing increased psychological adjustment problems in bullied children. More specifically, Hawker \& Boulton, [228] performed metaanalysis on cross-sectional studies of victimisation in schools, examining depression, loneliness, generalised and social anxiety, and global and social self-worth. Victimisation was most strongly related to depression, least to anxiety, though still significantly correlated with being bullied. O1weus also followed up children to age 24 , where he found a similar pattern of adjustment problems, with higher rates of depression, anxiety, suicidal thoughts and low self-esteem in those previously bullied compared with their non-bullied peers [230].

Cross-sectional studies can raise the issues clearly by focussing upon the later experience of psychiatric illness. Gladstone et al., [231] found over a quarter of both men and women presenting to an out-patient depression clinic reported severe and traumatic bullying at school. Many patients also had anxiety, including social phobia and agoraphobia; they also commonly reported a variety of other child adversities. Fosse [232] found about half of her new psychiatric patients reporting being seriously bullied at school, though in a relatively small sample. She found a doseresponse relationship, with more symptoms in those who had suffered more bullying.

In a large two-stage survey of about 3,700 Norwegian students in school aged 15/16 and 18/19, Lien et al., [166, 232] found $12.4 \%$ of boys and $11.7 \%$ of girls aged $15 / 16$ had been bullied sometime, and $3.8 \%$ of boys and $2.7 \%$ of girls had been bullied at least weekly. At age 18/19 only $4.7 \%$ of boys and $4.3 \%$ of girls had been bullied in the three years since the previous enquiry, and only $1.1 \%$ of boys and $2.0 \%$ of girls in the previous year. These diminishing prevalence figures were statistically significant. Both internalised and externalised psychological symptoms, and also many somatic symptoms, were far more common among those bullied than their peers, with varying significant ORs even when adjusted for other stressors [166]. There was some selection bias, especially for the later sample due to a low response rate from those who had left school. It was not truly a longitudinal study but two cross-sectional studies with overlapping populations.

In a Danish cohort of 12,270 men born in $1953,38 \%$ recalled some bullying in childhood, those bullied showing more depression and difficult peer relationships as adults, increasing as the degree of bullying increased. The association diminished when parental mental illness and social class were taken into account, but was still significant [233]. Those of low SES tended to have experienced more bullying than others. Another Danish longitudinal study of a 1990 representative population sample produced similar results: $44.3 \%$ had been bullied at school and this was highly correlated with both depression at age 27 and SES. The effect of bullying was to be worse on children of low SES, and they also tended to experience more bullying [234, 233]. The authors postulated that children from families of low SES tend to have fewer resources to call on to resist aggression, within themselves, the family and society.

For a Finnish cohort of over 5,000 born in 1981, frequent victim status at age 8 years among females independently predicted psychiatric hospital treatment and use of antipsychotic, antidepressant, and anxiolytic drugs. Among males, frequent bully-victim and bully-only statuses predicted use of antidepressant and anxiolytic drugs. Generally boys are bullies and girls are victims, and frequent victimization among girls predicted use of anti-depressants even when controlling for baseline psycho-pathology [165].

However, the Norwegian cohort of 2,464 adolescents reported by Undheim et al., [164] did not find any variation in risk according to SES or ethnicity. But bullied children were excessively from families with social problems, had lower social acceptance and few friends, low self-esteem, and emotional and behaviour problems. These victims of bullying were more likely to have depression and anxiety as adolescents, whereas children who bullied showed more delinquent and generally aggressive behaviour.

As stated in Section 7, there is good evidence that preventive programmes in schools can work, through measures to change the whole culture of schools, involving all teachers and all children, parents, and even sometimes the wider community. They should be more widely introduced [168].

There are relatively few papers from the cohort literature on the impact of parental mental illness on children, though there is an important literature elsewhere. It is relevant to several sections of this review, but is dealt with in Section 11 on parenting. Here it is sufficient to say that parental mental illness may often be one factor among several which have an impact as 'multiple adversity', may affect the quality of parenting, may be involved where there is child abuse, parental conflict, separation and divorce, and may lie in the background of child behaviour problems and school failure.

\section{Child Physical Abuse, Sexual Abuse, Neglect and mal-Treatment}

\subsection{Introduction}

There is a very substantial literature on child abuse in relation to later psychiatric disorder, amongst many other outcomes, and the output of papers has increased very substantially in recent years. There have now been many reviews examining various aspects of that literature and, in spite of the limited number of large-scale prospective studies they broadly agree on the overall picture [214, 235-237]. Child abuse is common wherever it has been measured, though figures vary and cover a wide range. It is difficult to know how much of this variation is due to differences and inadequacies of research method, and how much might represent 
differences between communities and cultures, which there must surely be [238, 239]. But substantial proportions of children and young people are apparently subjected to serious verbal, physical and sexual abuse, severe mal-treatment and neglect in every community, and it is only recently that authorities and community leaders have been willing to acknowledge it (eg [174, 240-243].

There are obvious problems of method: retrospective data from adults or even adolescents is always suspect and cannot easily be validated, though recent work has suggested that they are more reliable than previously thought. Most reviews conclude that most published papers lack methodological rigour and make a very limited contribution to our further understanding. Inevitably "More research is needed"! However, prospective data in cohort studies are far from easy to obtain, as the process is threatening to participants, and could prejudice relationships in a long-term study [244]. Moreover, the members of the long-standing British and other cohorts were children at a time when child abuse was not really on anyone's agenda, and few data were attempted [245]. The later British cohorts and the New Zealand ones are exceptions.

There is also a problem of definition and inclusiveness which also renders many studies of limited value [246]. Although this issue was given some prominence in the early 1990 s, a later editorial by Cicchetti and Manly in 2001 complained that there had been little progress [247] Variability of terms, age ranges and definitions is still characteristic. Some studies have used very broad definitions combining all forms of abuse, neglect or mal-treatment. For sexual abuse, some have included verbal abuse, others restricting their study group to court-verified cases of abuse, or proven penetrative sexual intercourse [245, 248-250]. The difference between the two ends of this spectrum is so great in the nature of the experience and their likely impact, that it is essential that studies divide them into clear sub-groups [251]. A helpful discussion of the problems of recording physical and sexual abuse of children was published by Fergusson et al., in 2008 [252].

However necessary various categories are for research, they mask the continuous nature of the spectrum of abuse, and the variability within any operational sub-groups. It is also to be expected that there is great variability according to the age of abuse (eg [241], the frequency of abuse, the length of time it continued, and the relationship with the abuser [253]. And, of course, as already discussed, there remain problems of variable definition and measurement of mental health outcomes.

It is not surprising, therefore, that results of studies link reports of child abuse to many very different adult outcomes, social, educational, behavioural and psychological. An early review (1993) of studies of physical abuse by MalinoskyRummel and Hansen [254] found associations with somatisation, anxiety, depression, hostile attitudes, paranoid ideas, psychosis and dissociation, as well as more violent behaviour as adults, and the same conclusions have been common ever since. This non-specific nature of associations is particularly characteristic of the literature [194, 214, 238, 239, $241,255]$, and it emphasises the seriousness of the impact, appearing to affect the whole of life for many abused people.
However, it must also be remembered that there are survivors who appear to have coped largely with the trauma, and live psychologically and socially effective lives [256-258], though some of these may nevertheless have nonpsychological ill-health which has also been found to be increased in the victims of child abuse [259].

If outcomes are varied and non-specific, so, it seems, are the putative causes. No one type of child abuse, however defined, stands alone, but should be seen in the context of family values, behaviour, conflict, socio-economic circumstances, parental illness, and so on, all of which may also change throughout the period of childhood. Most research has focussed upon one or more types of mal-treatment or adversity, though often acknowledging the problem. Recently, researchers using the USA National Co-morbidity Survey Replication of 2000-2001 have seriously addressed the issue and demonstrated that adversities are often clustered and few ever appear alone [193, 194, 259]. This is important to recognise when considering all the more limited associations discussed below. Another facet is connected which also is rarely addressed - the inter-generational transmission of abuse within families, examined very recently by Noll et al., [260]. They found higher risks of mal-treatment and adversity across three generations, but again, in a rather non-specific fashion.

\subsection{Frequencies and Causes of Abuse}

Some reviews have concluded that child physical abuse has been experienced by $10-31 \%$ of men and $6-40 \%$ of women; and child sexual abuse (CSA) by $3-29 \%$ of men and $7-36 \%$ of women [239], but the very wide ranges make it difficult to make general statements. Other reviews have suggested that $10-25 \%$ of women, and $2-10 \%$ of men experienced abuse as a child [251]. In the USA it is officially recorded that 16 per 1000 children are subjected each year to mal-treatment, and about 2.4 per 1000 are subjected to sexual abuse, about half of these involving genital penetration. Other figures available are very conflicting; women's retrospective reports give a very wide range, but often much higher figures [238]. The USA Centres for Disease Control (CDC) 2009 telephone survey of Adverse Childhood Experiences in five States had just over $50 \%$ response rate. Of 26,229 respondents, $17.2 \%$ of women reported child sexual abuse, and $6.7 \%$ of men. Data for the USA from CDC in 2009 suggest that these also are likely to be under-estimates [261].

In the USA, of 506 boys from Pittsbugh followed up from age 13 to 19,105 had child abuse before age 18 substantiated from official agency records; this was mostly physical abuse and neglect and almost all from within the household [262]. In another USA cohort of working class / lower middle class white children aged 5 and followed up at $9,15,18$ and 21 , recorded abuse before age 18 in $15.5 \%$ of women and $12.3 \%$ of men; sexual abuse was recorded in $5.9 \%$ of women and $1.1 \%$ of men [263]. In Christchurch, New Zealand, members of the birth cohort at age 18 reported retrospectively on sexual abuse experienced. Overall $17.3 \%$ of women and $3.4 \%$ of men reported sexual abuse: $12 \%$ of women and $3.4 \%$ of men reported non-contact abuse; $12.6 \%$ of women and $2.8 \%$ of men reported sexual contact not involving attempted intercourse; $5.6 \%$ of women and $1.4 \%$ of men reported attempted or completed intercourse [251]. 
May-Chahal and Cawson in 2005 [242] reported a representative population survey of 2,869 young adults in the UK who were interviewed by trained interviewers. Over $90 \%$ said they came from a warm and loving family background, but $16 \%$ had experienced serious mal-treatment: contact sexual abuse $11 \%$ ( $16 \%$ women and $7 \%$ men); physical abuse $7 \%$ ( $12 \%$ women and $17 \%$ men); emotional abuse $6 \%$; absence of care $6 \%$; absence of supervision $5 \%$. A UK national study in 1998 [264] was replicated in 2009 and did find some hope in reduced levels of mal-treatment reported, but they were still high [174]. They interviewed parents or guardians of 2,160 children under age 11;2,275 children aged $11-17$, and 1,761 young adults aged $18-24$, with the following results:

All severe mal-treatment: $5.9 \%$ of $<11 \mathrm{~s} ; 18.6 \% 11-17 \mathrm{~s}$; $25.3 \% 18-24 \mathrm{~s}$. Severe physical abuse: $1.2 \%$ of $<11 \mathrm{~s}$; $6.9 \% 11-17 \mathrm{~s} 11.5 \% 18-24 \mathrm{~s}$ Contact sex abuse: $\quad 0.5 \%$ of $<11 \mathrm{~s} ; 4.8 \% 11-17 \mathrm{~s} 11.3 \% 18-24 \mathrm{~s}$

Severe mal-treatment inflicted by a parent or guardian was $5.0 \% ; 13.4 \%$ and $14.5 \%$ respectively. Girls suffered generally more than boys except in physical abuse in the $<11$ s; overall mal-treatment in the $11-17 \mathrm{~s}$ was the same for both. Sexual abuse was experienced far more by girls: $0.8 \% ; 7.0 \%$ and $17.8 \%$ respectively. Most perpetrators were males known to the child, but $65.9 \%$ of contact sexual abuse was by peers.

It is widely believed that the figures reported in most studies are a substantial under-estimate of the real figures, but it is very difficult to know, and there has been some work to validate measures which gives a degree of confidence in the results $[265,266]$. We must conclude, however reluctantly, that it is very common.

This review was commissioned to examine the consequences of abuse (as a determinant of mental illness) and research on possible causal factors of abuse, risk factors and processes cannot be fully encompassed. However, it may be helpful to refer to one excellent research paper by Sidebothom et al., in 2006, prefaced by a review of the literature from which they concluded that four principle risk factors in parents emerged: young parental age; low educational achievements; adverse childhood experiences, particularly parental exposure to abuse or neglect in their own childhood; and parental psychiatric history, including a history of alcohol or drug abuse. They provide many references [267].

Their own study used the ALSPAC cohort (n 14,256) in SW England. The terms of reference for the cohort permitted contemporary collection of many data, and they had access to official records of $2.1 \%$ of children investigated for maltreatment and of those then placed on the Child Protection Register. These were relatively hard data on mal-treatment of children, but the authors were aware that not all maltreatment will have been recorded. Briefly, mal-treatment thus defined was highly associated with parents who were very young ( $\mathrm{x} 3$ ), had low educational attainment (x5), had a psychiatric history before pregnancy $(x>2)$ or had a history themselves of child abuse $(x<2)$. But the highest risks were found with indicators of deprivation (OR 11.02 for those on the Child Protection Register, controlling for parental factors). Single parents and re-ordered families were also at high risk of being Registered, as were, more surprisingly, children of low birth weight. Thus a wide range of factors contributed to increased risk to the child. From the same cohort, children of abused mothers had an increased risk of experiencing negative life events between ages 4 and 7 years, including changes in family composition, separations from parents, 'shocks and frights', and physical assaults [258].

\subsection{Consequences of Abuse}

\subsubsection{Multiple Effects and General Psycho-Pathology}

Published work in the last six years has greatly broadened our knowledge. As mentioned above, the relationships between abusive experiences and later psychological distress and psychiatric disorder is generally very non-specific, encompassing also almost every social ill. Meta-analysis is very difficult to perform convincingly when the data are so variable. Neither Neumann et al., [215] nor Rind et al., [268] solved the problems, but their common broad conclusion that virtually all psychiatric outcomes were associated, with small effect sizes, is probably not prejudiced. The recent large-scale study from Victoria, Australia followed up 2,688 children recorded officially as having been sexually abused between 1964 to 1995 and a random control group from the electoral register and matched for gender and age group $[241,269]$. They examined all categories of disorder using a public Psychiatric case Register, and found all of them with substantial associations (ORs between 2.13 and 5.94) with sexual abuse histories. This included psychosis (though this gave the smallest OR) and personality disorder. The one exception was eating disorders.

The NCS Replication in the USA has already been mentioned [193, 194]. They found little specificity for particular abuse experiences for classified disorders; indeed most abuses were associated statistically with almost all classes of disorder (psychosis being largely an exception). They also showed that most abuses were related to the persistence of disorders through adult life, but only modestly. They concluded that the overall statistical associations of child abuses with adult disorders applied largely to onset rather than persistence. Maniglio [214] in his excellent review of 14 previously published reviews came to the same conclusion: that survivors of sexual abuse were significantly at risk of a wide range of medical, psychological, behavioural and sexual disorders, though frank psychosis seems to be excluded from this. He wrote that child sexual abuse should be considered as a general, non-specific risk factor for psycho-pathology in general.

However we should not forget that not all those suffering child abuse are afflicted later. Collishaw et al., [258] in 2007, followed up the famous Isle of Wight study population of children from 1964, most recently when they were aged 44-45 years $(n=378) .10 \%$ of respondents had reported repeated or severe physical or sexual abuse as children, but almost half $(45 \%)$ of these showed no psycho-pathology over their adult lives to their mid forties. The numbers are small but the lesson needs to learned. All other problems of behaviour examined were less frequent on the 'resilient group' than the others. 'Resilient' people commonly reported at least one parent as 'very caring', and peer relationships in adolescence, quality of adult friendships, and stability of adult love relationships were all strongly related to resil- 
ience. DuMont et al., [270] in 2007, also found almost half of abused and neglected children in Mid-Western USA were resilient with no psycho-pathology in adolescence, and about one third in early adult life.

Some studies are fairly general and encompass a wide range of psychological measures, especially from the birth cohorts, many appearing in several sections here. In a paper from 2008 [252] the team from the Christchurch cohort looked broadly at 'adjustment' in early adult life, encompassing depression and anxiety disorders, conduct disorders and anti-social personality disorders, substance misuse, suicidal ideation \& suicide attempts. Apparent correlations with child physical abuse (CPA) mostly disappeared after adjustment for family, social and individual factors, though leaving harsh or abusive physical punishment with a 1.5 times risk of mental disorder by age 25 . They suggested that they are family contextual. But correlations with child sexual abuse (CSA) remained after all adjustments with a 2.4 times risk of disorder by age 25 . Like other researchers (eg Fisher et al., in 2010 [271]), they found both types of abuse were associated with most negative factors, and were themselves somewhat correlated. They estimated that CSA accounted for about $13 \%$ of psychiatric disorder by age 25 in their population; CPA only about $5 \%$.

\subsubsection{Psychotic Disorders}

As noted in the previous paragraphs, schizophrenia and closely related syndromes have not generally been much associated with previous child abuse but the picture is not simple. Spataro et al., in 2004 [250] showed no difference from their general population controls, and Shevlin et al., in 2007 [272] using NCS data from the USA, found overall associations with psychosis only for physical abuse, though for rape also in men. They only had retrospective data. There have been two recent reviews of the evidence. Morgan \& Fisher, in 2007, [7] considered that most papers lacked methodological rigour and were therefore of very limited value, leaving any relationship of childhood trauma to psychosis controversial and contestable. There was modest evidence of an increased risk of 'psychotic symptoms' (not diagnosable psychosis). Bendall et al., in 2008, [8] reviewed 46 published studies and found only six of them useful. Three of these found a statistically significant association of childhood trauma with psychosis, and even these were of low power or had other limitations.

After reviewing what was published, Bendall et al., offered the suggestion that child trauma might be associated with atypical psychotic symptoms, but not recognised psychosis per se. This perhaps coud be linked with a Schurhoff and Laguerre et al., study in 2009 [273] of children at high genetic (familial) risk of schizophrenia. They found an association in these subjects of child trauma with psychotic features ('schizotypal dimensions') and suggested that there might be interaction between the trauma experience and the genetic predisposition.

The outstanding cohort study contributing evidence at variance with most of the literature is that of 2,759 individuals ascertained as having been sexually abused when younger than 16 years in Victoria, Australia and followed up into adult life with a matched control population [269]. In the abused subjects they found increased rates of psychosis in general (OR 2.1; CI 1.4-3.1; $\mathrm{p}<0.001$ ) and schizophrenic disorders in particular (OR 2.6; CI 1.6-4.4; $\mathrm{p}<0.001$. Penetrative sexual abuse had even higher rates in both categories, but, on its own, abuse without penetration showed no significance in either. Higher risks attached to abuse after age 12 , and more than one perpetrator.

Some confirmation of these results arises from a careful 2010 UK case-control study of 182 cases of adult psychosis on first presentation, and 246 geographically matched controls from Nottingham and London [271]. Using the Childhood Experience of Care and Abuse Questionnaire (CECA.Q) and other data collected in face-to-face interviews, cases recorded about three times the rate of severe physical abuse by the mother before the age of 12 , even after adjustment for confounders $((\mathrm{OR}=3.01,95 \%$ CI $1.02-8.89$, $\mathrm{p}=0.046)$. Correlations with sexual abuse and neglect diminished below statistical significance when adjusted for maternal antipathy and physical abuse. These abuse categories were clearly not independent variables. There were no significant correlations relating to paternal behaviour. The age of most impact was the opposite of the Victoria study. The authors point out that numbers were small because the vast majority of cases $(86 \%)$ and controls $(93 \%)$ did not report any maternal physical or emotional abuse.

An illustration of the complexity of the situation comes from a paper comparing USA data from the NCS and British data from the National Psychiatric Morbidity Survey [274]. The authors examined the relationship between a summative index of 'number of traumatic experiences' and later psychotic illness, and found a progressive relationship - more traumas indicated a higher risk of psychosis. This helpful, but they also found differences between USA and UK populations in what particular traumas gave significant correlations with psychotic illness. Could cultural differences explain such findings?

Given that these are impressive studies with reasonable numbers and apparently reliable data, the results are not easy to interpret in the light of the rest of the literature. However, if there is a correlation between child abuse and psychotic illness, the direction of causal relationship must be considered. It is possible that children with early, but undetected signs of later psychosis, are more vulnerable to abuse by parents, especially in the light of the generally consistent evidence of behavioural, intellectual, language and motor abnormalities as precursors of later psychotic illness. (see Sections 3 and 5).

\subsubsection{Depressive and Anxiety Disorders}

In 2009 , in a systematic review of published prospective studies, Weich et al., [237] found that poor relationships at home, especially mal-treatment in childhood, was associated with later depression in eight out of ten cohorts with adequate data for women, and six out of ten for men. Anxiety was associated in both of two studies while post-traumatic stress disorder (PTSD), (which can be considered an anxiety disorder) [255], was associated in two out of three studies. A wider review by Nurcombe in 2000 [238]) found PTSD especially associated with sexual abuse, but there were many other negative outcomes too. Fergusson et al., in 1996 [235, 
$251,274]$ reviewed other reviews and concluded that child sexual abuse was always associated with significantly higher risk of psychiatric disorder. From the Dutch NEMESIS study, using retrospective data in a large population sample, the only important measured pre-cursor of the incidence of adult depression in the first year of follow-up, appeared to be 'childhood trauma', particularly 'neglect' and 'childhood adversity' .

Weiss et al., in 1999 [236] thought that data from many studies suggested that the greater the severity, frequency and duration of sexual abuse, the greater the risk of subsequent depression, a conclusion consistent with reasonable expectation. Although findings relating to the age of abuse and the relationship of the abuser to the child were not generally clear, it was likely that the most damaging situation was father-daughter incest. Meta-analyses are not easy to justify in this field where definitions and methods are so variable, but Neuman et al., in 1996, Rind et al., in 1998, and Paolucci et al., in 2001 [215, 268, 271, 275] all concluded that child sexual abuse was significantly related to depression and PTSD, among other things, in the data they had analysed.

More recent reviews have confirmed the substantial risks of depressive and anxiety disorders related to child abuse. As already noted, Maniglio in 2009 [214] found the same in his review of reviews. In a particularly important review Weich et al., in 2009 [237] focussed exclusively upon the output of longitudinal cohort studies, finding 23 papers on 16 cohorts following subjects for from 10 to 37 years. The most robust findings related to severe child abuse and neglect which were predictive of later depression in all but one relatively lowquality study. All methods of ascertainment of severe abuse and neglect (parental interview, observation, child report and court records) were predictive of depression. The results of these studies were independent of socio-economic factors and, in some cases, of factors such as child behavioural problems and family stress that might have been acting as mediators of the association between abuse and mental health outcomes. Studies with data on childhood abuse or neglect found this also to be associated with PTSD, even after controlling for potential confounders and effect mediators. These findings are consistent with evidence that childhood sexual abuse, whoever the perpetrator, is associated with an increased risk of most forms of non-psychotic mental illness in adulthood. The authors comment that, in spite of our conventions of taxonomy, anxiety, depression and PTSD share phenomenological and aetiological similarities and commonly occur simultaneously.

Individual cohort studies have confirmed these findings. In the Dunedin birth cohort, adult-onset major depressive disorder had a highly significant association with child sexual abuse (ORs over 2) [93]. Child sexual abuse experienced by the mothers of the very large $(>14,000)$ British Bristol pre-birth cohort of 1991/92 (ALSPAC) was related to higher levels of depression and low self-esteem, though effect sizes were only about 1.4. They also had less satisfaction in relationships in general and with their partner in particular, and poorer communication skills, though no less enjoyment of their child. Higher frequencies of behavioural and emotional problems in their children appeared to be mediated by mother's mental health problems, especially anxiety and de- pression, and related to their experience of child sexual abuse. Abused women were very frequently pregnant in their teens $(26 \%)$, had a variety of parenting problems, and, especially if sexually abused, were more likely to mal-treat their own children [255].

The only data available from the British 1946 birth cohort are scores from the Parental Bonding Instrument (PBI) given retrospectively at age 43, and the Psychiatric Symptom Frequency scale (PSF) given at the same age. High affective symptom scores (largely depression) were associated with high PBI scores for parental control, and low scores for parental care. Low levels of depression were associated with low control scores and high care scores. People reporting a history of mistreatment also gave low care and high control scores for their parents, most of all for fathers where physical or sexual abuse was reported. The link with depression is implied [245, 276].

In the Christchurch cohort, child sexual abuse was strongly associated at ages 16 and 18 with major depression, anxiety disorders, conduct disorders, substance abuse and attempted suicide, with ORs ranging from 2.7 to 11.9; the more severe the abuse, the higher was the risk [235, 257, 120]. At age 21, panic attacks and panic disorder were associated with earlier physical abuse, and especially sexual abuse [277]. However, there was relatively poor correlation of these retrospective reports of abuse at age 18 and similar reports at age 21. Evidence from other cohort data collected during childhood suggest that this retrospective abuse data included many false negatives, perhaps $50 \%$, but few false positives, and the relative risk remained stable. Odds ratios of just under 2 for psychiatric disorder related to sexual abuse remained after adjusting for many other childhood factors including physical abuse, social disadvantage, family dysfunction, impaired parenting and parent psychopathology [26].

Serious physical violence by parents in Christchurch was associated with later depression and other mental health problems in the children, but the significance mostly disappeared when adjustment was made for confounders - the conclusion being that more general family conditions underlay the violence. But father-initiated violence was still associated after adjustment with conduct disorders, anxiety disorders and property offending, in boys and girls [278].

A cohort in the USA followed 5-year old children for 16 years, and found strong associations of physical abuse with later major depression and PTSD in both men and women, and even stronger associations for sexual abuse, but numbers were very small [263]. Similarly, small numbers reduce the value of associations with depression and suicidal behaviour in an up-state New York cohort [279].

In San Diego, USA, a different approach permitted large numbers to be studied, but in a non-representative population. Four retrospective birth cohorts were established for birth years from 1900 on, from the clients of a large health management organisation (HMO), of whom about $81 \%$ are seen in any one year. About $65 \%$ responded. $25 \%$ of women and $16 \%$ of men reported contact sexual abuse in childhood. Within all cohorts there was a progressive increase in lifetime depression, suicide attempts and a range of other psy- 
cho-social problems [280]. However, there are no data presented as to how these cohorts relate to any general USA population.

Yet another approach was adopted in a USA Mid-West metropolitan area where cases of child abuse substantiated by the courts from 1967 to 1971 were matched with controls, though only in $74 \%$ of cases, and controls had unknown histories of abuse. They were followed up 25 years later. The cohort encompassed a wide age range, which added more problems for analysis. Abuse victims, male and female, had significantly more anxiety and depression and anti-social personality disorders, but significance disappeared when adjustment was made for stressful life events. However, it seems likely that life events were more common in those abused. Relationships were seriously affected in many of the abused victims $[248,281]$. Overall, these results cannot be given too much weight except that they confirm many others.

A similar method, but perhaps more satisfactorily controlled, was used in Victoria, Australia, in identifying 1,612 children with medically validated sexual abuse, and following them with controls into mid-life on the Victoria Psychiatric Case Register (which does not include private medical services). Significant relationships were clear for personality disorder (OR 5.47), PTSD (OR 5.56; more for women) other anxiety disorders (OR 2.67) and major or minor affective disorders (OR 2.07; more for women) [250]. The 1958 British cohort is now also in mid-life. Clark et al., in 2010, [282] examined all the data for the 9,377 people who responded in 2003 (59\% of the surviving sample) for the relationship between childhood abuse (collected retrospectively at age 45), earlier measures of childhood adversity, psycho-pathology in adolescence and early adulthood, and depression and anxiety at 45. When adjusted for SES, childhood adversities were associated with adolescent, early adult and mid-life psychopathology and most associations did not attenuate with age. Mid-life depression and anxiety were associated with cumulative adversity, sexual or physical abuse and illness in men and women.

Researchers have continued to analyse the data set from the large cross-sectional, retrospective, USA National Comorbidity Study of 1990-1992. Subjects recalling child sexual abuse ('rape' or 'molesting') had higher rates of generalised anxiety disorder (GAD), panic attacks, panic disorder, and post-traumatic stress disorder (PTSD) but the strongest association was with the number of diagnoses, which may support the view that they are all related phenomena. The association of most categories was attenuated when controlled for parent and peer relationships, but major depression (MDD), dysthymia and bipolar disorder remained significant even when divorce was controlled for [283, 284].

Those data are now 20 years old and presumably no longer represent the American population as once they did. The NCS Replication collected new cross-sectional data in 2001-2003. Cougle et al., in 2010, [285] found physical abuse to be especially related to PTSD and specific phobia, but sexual abuse was associated with social anxiety disorder (SAD), PTSD and personality disorder. Among men, both forms of abuse were associated with SAD and PTSD. In the Netherlands Study (NESDA) [286] depression and anxiety, and their chronicity, were associated with more and more frequent childhood abuse, and similar relationships have been reported by many others in recent work [287-292].

Disorders of depression and anxiety, including PTSD, are the most commonly and consistently recorded increased risks for adults abused in childhood.

\subsubsection{Suicide Ideation and Suicide Attempts}

It is, perhaps not surprising, given the high rates of depression in those who suffered child abuse that suicide ideation and attempts are more common than in their non-abused peers, but the association is never a simple one. Reviews by Mina \& Gallop in 1998 [246], and Beautrais in 2000 [37] both concluded that child abuse was one of many factors associated with later suicide behaviour. Paolucci's metaanalysis in 2001 specifically identified sexual abuse as a risk factor, and Brezo et al., in 2008 [275, 293] found the risk highest following repeated sex abuse. Enns et al., in 2006 [199], in the large population study in The Netherlands, NEMESIS, (> 7,000 aged 18-64) found childhood neglect, physical or psychological abuse strongly associated with new suicide ideation or attempts in a three-year follow-up period, but not sexual abuse. However, when they looked at data for suicide ideation and attempts throughout adult life there were very significant associations with reported sexual abuse (ideation: OR 2.33; CI 1.58- 3.43; attempts: OR 2.62; CI 1.36-5.03)

The USA National Co-morbidity Survey (NCS) of 19901992, and its Replication in 2001-2003 found much broader associations with most forms of abuse and maltreatment, but violent sexual abuse gave the highest risks [284, 294]. Their estimates of the population attributable fraction for suicide ideation were $16 \%$ for women and $21 \%$ for men; and for suicide attempts, $50 \%$ for women and $33 \%$ for men. Of course, causation is not proven by association, but any alternative is currently less convincing. However contentious these figures may be, they must surely give a fair indication of the huge importance of childhood abuse even in just this one effect.

Longitudinal studies have less commonly had data on abuse and suicide. Weich's 2009 review of cohort studies found only two with appropriate data; they found strong associations with suicidal ideation and attempts. The Victoria study data particularly high-lighted the high rates of suicide and accidental (presumed) fatal drug overdose [295]. A related phenomenon - self injurious behaviour has also been linked to child abuse in two reviews from 2008 and 2009 [296, 297], though in the latter case, the authors had little confidence in respect of child sexual abuse. In a 2010 review [298], Perepletchikova \& Kaufman were more confident in finding violence towards both self and others linked to earlier experience of abuse. Most dramatically, one study showed suicide attempts in abused boys fifteen times more frequent than others.

\subsubsection{Other Outcomes}

Personality disorders have been associated with childhood abuse in some studies with a wide scope. Cutajar et al., [241] found a very significant relationship in the large Victoria study, giving an OR of 5.47 (CI 3.30-9.08) for PD overall, but all types gave somewhat similar results. Associations 
with borderline PD was almost exclusively in women (x7 controls), but anti-social PD was predominantly a male phenomenon. Perhaps stereotyping and labelling by expectation played a part? The authors felt that the study provided strong confirmatory evidence for CSA being a risk factor for developing severe personality disorders.

Fossati [299] in his meta-analysis found no support for this relationship, but Caspi et al., [300], in a case-control study found significant excess of obsessional compulsive disorder and OCPD in patients who had experienced contact sexual abuse as a child, but not otherwise.

Eating disorders seem to have attracted very little attention in this literature and what there is has provided conflicting and very uncertain evidence, though from the same population. In the large and convincing follow up study of 2,688 sexually abused children and controls in Victoria, Australia, virtually all categories of disorder were significantly associated with abuse, including even psychosis, but not eating disorders, which showed no correlation at all [241]. From the same population but a different sample, another Victoria cohort study focussed specifically upon eating disorders, 1,936 adolescents were observed from 1992 to 2003. Self-reported child sexual abuse before age 16 was ascertained at age 24 , but eating disorders had been identified during teenage years. They did find one strong and significant relationship - for bulimic syndromes related to more than one experience of sexual abuse [301].

There are serious problems of definition and measurement of eating disorders as well as child abuse and the usual epidemiological issues [302], and numbers tend to be very small for conclusive studies. Palmer et al., in 1990 [303] collected a sample of 158 women (aged 15-49, mean 24) over a number of years, who were questioned carefully about child sexual abuse and later adverse sexual experiences, by self-report questionnaire followed by an interview. Virtually equal numbers had anorexia nervosa and bulimia. About a third reported child sexual abuse, $80 \%$ contact abuse, though a minority had experienced full penetration. Though these seem high rates, the authors could not adequately compare them with rates for psychiatric conditions in general or other populations. They concluded that, though the hypothesis that child sexual abuse was a causal factor in later eating disorders was compatible with the evidence, it was unlikely to be specific, and anything more certain would have to await further research.

Recently, a relatively large sample $(753$, mean age $~ 29)$ of women with eating disorders has been reported in a study that examined the relationships with concurrent PTSD, and possible precursors. $13.7 \%$ had PTSD, proportionately more for anorexia than bulimia. The most frequently reported traumatic events by those with both eating disorders and PTSD were sexually related traumas in childhood (40.8\%) and in adulthood (35\%). But it is hard to disentangle these variables and draw any conclusions about child sexual abuse and eating disorders per se [304].

Quite a number of studies have focussed not upon psychiatric disorders but more ambiguous symptoms of emotional and psychological distress and adjustment. Jumper ([305] published a meta-analysis in 1995 of the relationship of child sexual abuse to adult psychological adjustment as measured by psychological symptoms, depression and selfesteem. Results suggested an increased risk (not surprisingly) but any interpretation is prejudiced by the heterogeneity of studies. Following up 907 youths from a community sample in Rochester, USA, from age 14 to 31 with 14 serial assessments, Thornberry et al., in 2010 [306] found specific relationships of childhood maltreatment with internalising problems, but adolescent abuse had stronger and longer effects on adjustment.

In Watson and Halford's 2010 study [307] of 1,335 Australian women aged 18-41, reported child sexual abuse had the highest risk of adult relationship problems when perpetrated by a family member. Other studies report more adolescent ADHD [308], adult psycho-social difficulties [309], emotional and behaviour problems [298], and psychologically generated seizures [216]. Given the overwhelming finding that whatever adverse or pathological psychological conditions are investigated, they seem to be associated with child abuse, these must simply be added to the melange.

\subsubsection{Conclusion}

Acknowledging the vagaries of research results, we must believe that child physical abuse and child sexual abuse are both common in western societies (and elsewhere). In spite of inherent methodological problems, there is now a great deal of evidence from many studies in many different communities, that child abuse, and especially child sexual abuse, has a very powerful impact on a majority of victims and the consequences are legion and important [239]. They include major psychiatric disorders, personality disorders, high risk life-styles, self-destructive and violent behaviours, antisocial behaviour, problems with relationships, impaired capacity for parenting, physical illness, early parenthood, and others. There are extremely important knock-on consequences for others, especially their own children, and for society in general (eg. [310, 260]).

However, we must not forget that many children who are severely abused grow up into apparently healthy and untroubled adults. This is no reason for complacency but is an opportunity to explore the origins, processes and influences that produce such resilience, that we might learn how to improve the resistance of others to childhood trauma. But it remains far more important to find ways of diminishing abuse.

For treatment, claims are made for the effectiveness of cognitive behaviour therapy, but the huge importance of prevention should also concentrate our minds. However, the prevention of child abuse is far from easy, and there do not seem to be many ideas among professionals or politicians. A variety of amelioration strategies has been reviewed recently by Lalor and Mc Elvaney in 2010, [213] but the quality of research published is not impressive. We need some new thinking if we are to save the present and next generation of children from this scourge. Few researchers have examined this, though Collishaw et al., in 2007 in the UK [258], and DuMont et al., in 2007 in the USA [270] have set examples, and much more might be done. There are pointers to factors which are not really surprising - good relationships with parents, or at least one parent, and peers at all ages, are most likely to be important in fostering resilience and stable per- 
sonalities. The corollary opposite is that unloving, conflictual or broken family relationships reduce the capacity of children to resist trauma.

Both prevention and amelioration are challenging, but there are many possible strategies; several are discussed by Lalor \& McElvaney [213]. Mostly, in Western societies we are doing little in a concerted, logical, evaluated way, though many small scale initiatives can be cited. In the UK the very recent (2011) major surveys of the appalling frequency of abuse $[174,240]$ should stimulate serious, national and prolonged action, but previous action or inaction by governments does not offer much hope.

\section{Parenting, Parent-Child Relationships and Parent Psychological Disturbance}

\subsection{Introduction}

Parenting - its style and quality - has not been a subject of study for very long; there were no validated measures until relatively recently, and it was not generally conceived of as an issue in research. So measures were not generally applied to the old, long-standing cohorts, such as the 1946 and 1958 British cohorts, but there is hope for the future in the 1970 and the Bristol ALSPAC cohorts. For most studies, therefore, it was only possible to collect data retrospectively; for example, the 1946 cohort used the Parental Bonding Instrument (PBI) at the age of 43 , so issues of recall are prominent [245].

Measures such as the PBI are now available, and to some extent now determine the aspects of parenting examined. In particular, they record degrees of 'care' and degrees of 'control' experienced by a child from each parent, and this has lead to some common stereotypes, especially 'affectionless control', which has often been associated in research with depression in adulthood.

Creating instruments to measure 'parenting' and thus identifying it as a separate variable, does not, of course, make it a psycho-socially independent entity. It is clear that it must relate closely, indeed, to overlap with family structure, inter-parental conflict, divorce and separation, child abuse and neglect, as well as aspects of child behaviour, and aspects of material circumstances. It is very artificial to measure it and analyse it as a separate entity, but without doing so there can be no clarity on its contribution to the whole family picture.

Parenting styles are also very much subject to cultural variation, norms and pressures. What in one culture is acceptable daily discipline is seen in other cultures as violence or abuse. And these attitudes are subject to progressive change in most societies; historical accounts reveal very different expectations of mother-child, father-child, and mother-father relationships, from those in our own current cultures and sub-cultures. And this change is rapid enough for it to affect the long-term cohorts currently available for study, so that many aspects of family life, including parenting, cannot be interpreted without understanding attitudes and expectations in the $1940 \mathrm{~s}$ and $50 \mathrm{~s}$, or whatever period is in focus.

So, there are difficulties of measurement, difficulties of analysis in relationship with other variables, and difficulties in interpretation, as well as only relatively recent data. Nevertheless, evidence is gradually being accumulated on the effect of parenting style on child, adolescent and adult relationships, behaviour and illness, which suggests that it has a significance in the family which cannot be ignored if children in the future are to be given the childhood experience they need for healthy and happy adult lives.

\subsection{Parenting Style and Child-Parent Relationships}

There have been several reviews. In 1999, Weiss et al., [236] looked at eight studies, and concluded that poor parenting was associated with later depression even in the absence of child abuse, though it is often correlated with child abuse. Buist in 1998 [249] examined relationships between mother's experience of abuse as a child and her own parenting problems, though almost every study reviewed had different problems or different ways of describing them. Parenting difficulties were more likely in mothers who themselves had been abused, but the risk factors were similar to those for abuse itself, and for post-partum depression, a major focus of this review.

A more recent review by Weich et al., in 2009 [237] examined only cohort studies. Earlier work had found only ten with relevant data for some parenting variable and data for later depression, though some cohorts were small - only four had $>1000$ subjects. Relationships at home were considered to have an important impact on later mental health. In particular they found mal-treatment in childhood was strongly associated with adult depression, in eight of ten studies for women, and six out of ten for men. But there were only four studies encompassing parent-child discord, and 'affectionless control' which were associated with later depression in three of them. The authors commented that most studies focus upon abuse and neglect, but that there is evidence that less dramatic problems of parenting are also important. Much more research is needed, however.

Their review of the literature on prospective studies again in 2009 was restricted to those where measures of relationships in the home were made during childhood, and there was at least ten years between that and measures of psychiatric disorder. By that time they found 23 papers relating to 16 cohorts, following up children for between 10 and 37 years. As one might expect, the most robust findings related to child abuse and neglect which predicted later PTSD in all, and depression in all but one study (which had some problems of method) - see Section 10. Associations with other forms of dysfunction in parent-child relationships were less consistent. Eight out of ten studies measuring aspects of family relationships (including quality of marital relationship) found positive associations with depression, but not all in the same groups. So conclusions are suggestive but not proven. However, where parenting was more dramatically negative, harsh maternal discipline and/or emotional unresponsiveness, the experience predicted more suicide attempts in the children as adults, as well as anxiety and PTSD. The reviewers still found too few, and too inconsistent studies of relationship quality to comment.

Focussing on parental bonding and adult psychiatric disorders, Lima et al., in 2010, [311] found only eight papers, of which only two reported longitudinal data. In spite of 
method inconsistencies, they thought that there were clear conclusions relating parent-child relationships to later mental illness, in both positive and negative ways, reported in conventional concepts. Parent-child interactions characterised by affective enhancement (affectional bonds) and low control (encouragement of autonomy) appeared protective of psychiatric symptoms in adulthood, whereas relationships characterised by affective impoverishment (neglectful relationships) and over-protection (intrusive behaviour) were apparent risk factors.

The PBI was used retrospectively at age 43 in the British 1946 cohort because no data had been collected during the earlier life of the cohort [245]. Self-reported neglect and abuse were significantly related to multiple health problems and diseases including depression. But low scores on the PBI 'care' scales and high scores on the 'control' scales, combined as 'affectionless control', were associated strongly with depression (measured on the PSF) at ages 36 and 43 [276]. Indeed, there were progressively higher symptom scores from high care / low control to low care / high control, and these associations were not removed by adjusting for social class, divorce, mother's neuroticism and other possible confounders. Those reporting abuse or neglect also gave low care and high control scores, especially for fathers, which, at least, has face validity. However, it also seemed that high levels of emotional support and an effective social network in adulthood could remove or reduce the significance of these characteristics of childhood [127].

A further follow-up of this cohort related retrospective data on the experience of parenting to the psychological well-being of women at age 52 [312]. The effects of parenting, both positive and negative persisted even into the sixth decade of life. As before, a high level of parental care was positively associated with all aspects of well-being, especially upon the quality of relationships in later mid-life. The effect of father's parenting was at least equal to that of the mother. The effect of personality (using the MPI) was interesting: the correlations between well-being and parental 'care' or 'non-engagement' were significantly affected by the personality of the offspring, whereas those between wellbeing and parental 'control' seemed to be direct and independent of personality features. The category of well-being 'autonomy' gave strange results, probably because of the unusual definition used in the Ryff Scales.

In the British 1958 birth cohort, relationships with parents were reported by adolescents at age 16 . Poor relationships with mother and father at 16 were associated with poor general health, mental health, and multiple problems and diseases at age 33. Specifically, poor parental relationships were associated progressively with worse mental health (MI data) at 33 for both mother and father, though rather more for father. However, no association gave a risk of more than twice those for good parental relationships [276]. On the positive side, high parental involvement with the child and interest in their education carried a particularly low risk of teenage child-bearing [187].

Work on the year 2000 wave of data collection for the 1958 cohort confirms previous findings [313]. There was an advantage over previous data collections in using the 12-item General Health Questionnaire (GHQ-12) as well as the MI, both validated instruments for mental health problems in adults. Relationship with mother and relationship with father were both predictive of mental health problems at age 42 as measured by the MI, results being very similar to age 33 . Both relationships were similarly predictive as measured by the GHQ-12, and there was a significant trend for both mother and father, with increasingly poor relationships resulting in increasing likelihood of mental ill-health. Adjustment for sex and social class attenuated the relationship a little, as did adjusting for the impact of the relationship with the other parent. In these analyses, as in those reporting the total number of health problems or diseases, relationship with father appeared to be slightly more influential than relationship with mother, but as in earlier analyses of health in general, both retained an independent effect in the final analyses.

In the British 1970 birth cohort various aspects of parentchild relationships, using a variant of the PBI, were recorded at age 16 for about 4,000 children. The Malaise Inventory was used at age 16 for mental health. Poor relationships with parents at age 16 were associated with poor mental health, general health and multiple problems and diseases at age 26. Good relationships at 16 were associated with good health at 26 . For mental health, poor scores at 26 were predicted by "Parents always nagging, moaning etc", and "Parents overprotective and fussy" for women, and by "Parents treat me like a child" and "Parents don't understand me etc" for men. "Parents loving, caring, look after me" predicted good scores [276].

The work on year 2000 data included the 1970 cohort and also confirms previous findings [313]. They also used both the MI and GHQ-12 and at age 16 they had used a more detailed questionnaire based upon the PBI. At age 30, results for the GHQ-12 and MI were almost the same. All five of the positive aspects of parental relationships covered by the questionnaire at age 16 were predictive in unadjusted analyses and those adjusted for sex and social class. Three (GHQ) or four (MI) remained predictive after adjusting also for depression at age 16, these data being available for this cohort.

For the six negative aspects of relationships in the questionnaire at age 16, all were predictive, increasing the odds of mental health problems at age 30 between $40 \%$ and $100 \%$. Adjustment of these analyses for sex, social class and teenage depression had only a marginal effect on the results; 'My parents are strict and bossy' became less significant related to MI scores and non-significant related to GHQ scores, and 'My parents are over-protective' became less significant related to GHQ scores and non-significant related to $\mathrm{MI}$ scores. The most predictive descriptor for both measures was 'Parents don't understand me / my motives'.

Interestingly, the rather general question at age 16, 'My parents treat me like a child', the most predictive question in unadjusted analyses and those adjusted for sex, social class and depression at age 16 , became virtually non-significant when adjustment was made for all the other questions. Presumably the more specific questions covered the same feelings about their relationship with their parents.

Stewart-Brown and Shaw in 2004 [276] not only reviewed all the relevant publications from the three British 
cohorts, but done some additional analyses on their data, incorporated into the summaries above. In spite of the many inadequacies of the data available, their overall conclusions were that parent-child relationships help to determine both mental and physical health in adulthood, and that the child's perception of their relationship with their parents is predictive of both in all three cohorts. This is independent of social class and adolescent mental health at the time of recording the relationships. In many cases, the father's relationship seemed particularly important. The size of the problem makes it an important public health issue.

These conclusions were confirmed by later follow-up of the British cohorts $[312,313]$. In the 1958 cohort, parental relationships progressively predicted mental health problems in adulthood (GHQ-12 and MI). Increasingly poor relationships with either parent were associated with increasing mental health problems at age 42 . In the 1970 cohort, positive items from the PBI predicted lower risk of mental health problems, whereas negative aspects predicted increased risk at age 30 . Odds of mental health problems were increased by around $50 \%$ in fully adjusted models. Interestingly, in an earlier analysis of cohort data, Stewart-Brown et al., in 2005 $[313,314]$ had shown a wide range of non-psychiatric illnesses at ages 26,33 and 43 , to be associated with poor quality parent-child relationships, as well as the expected abuse. Poor quality relationships with mother or father in the 1958 cohort, and a range of descriptors of negative relationships in the 1970 cohort predicted reports of three or more illnesses or health problems in adulthood.

In Victoria, Australia, they also used the PBI, at age 17, together with two psychiatric instruments, the Clinical Interview Schedule - Revised (CIS-R) and the Composite International Diagnostic Interview (CIDI) [315]. Low maternal and paternal care scores increased independent associations with depressive disorder by the age of 17 as recorded by both instruments (OR 2-3 for lowest quartile). High maternal and paternal control scores similarly predicted depression, but care seemed the more powerful predictor.

The 'Children in the Community' longitudinal study of 593 families in New York State, recorded ten types of parenting behaviour during child-rearing years, covering punishment, degree of affection, time given to a child, extent of supervision and effectiveness of communication. A paper by Johnson et al., in 2006 [316] focussed on personality disorder outcomes. All types of parenting, when negative, were associated with greater frequency of all types of PD, though varying somewhat in degree. However, $20 \%$ of all subjects were diagnosed with one type of PD or another, and these results might suggest the need for far better nuancing of measures. Gao et al., in 2010 [317] found that poor parental bonding was associated with later 'psychopathic personality' and that early separation made poor boding more likely. But the sample was small and the study only cross-sectional.

A much more recent British pre-birth cohort in Bristol (ALSPAC) with about 14,000 births from 1991/92, has a much wider range of relevant data from all ages, but, as yet, their subjects have barely reached adult life.. Parenting problems have been related especially to the mother's experience of child abuse, but these appear mediated by the mother's mental health problems [255]. This is consistent with the finding that mal-treatment of the children in the cohort (162 cases identified) was closely associated with three factors very young parents, parents of poor education, and parents with psychological ill-health [318]. All these might be expected to have a high risk of difficulties in parenting, but the direct links have not yet been published. It is, perhaps, not surprising that poor parental child-rearing has also been associated with adult offending and delinquency, as in the South London cohort [148].

In the Christchurch birth cohort they have demonstrated that less nurturant parenting is associated with very young mothers. Indeed, their provisional conclusion after complex analysis of many factors, was that the association of low maternal age with poor education and psycho-social outcomes could be largely explained by factors likely to increase the risk of poor parenting, including changes of parents, physical punishment, abuse, and parental history of offending and illicit drug use [319]. In a review by Nagin et al., in 1997 [320] it was concluded that both low level material resources and poor parenting were important in rendering very young mothers and their children more vulnerable. Child sexual abuse produced a higher risk of parenting problems with their own children, but abuse was also more likely where there was poor parental bonding or over-protection [251]. Suicidal behaviour was also associated with poor parent-child relationships [38].

On the positive side, paternal support, affection and nurturing in Christchurch were associated with significantly fewer mental health problems: close parent-child relationships may protect children even from the effects of earlier abuse [257]. Interestingly, in the Tampere, Finland cohort, closeness to the father appeared protective of later depression after divorce, but only for girls [124]. In a small USA study, warm and supportive relationships with parents was associated with better school performance, less risk-taking behaviour, and less teenage pregnancy [321]. In that study, adolescents involved with pregnancy usually had conflictual relationships with parents. In adolescence and early adult life, adjustment problems, poor school performance, low self confidence and unsatisfactory peer relationships were associated with 'disruption of skilful parenting', itself deriving from depression and demoralisation in the parents due to unemployment and severe economic stresses [180]. These are very likely to increase the risk of depression in the children, as is shown in a larger USA study, where maladaptive parenting had a high risk of later psychiatric disorder in the children, particularly anxiety and depression [122], as well as suicide attempts in adolescence and young adulthood.

There are several interesting papers which examine early parenting in the light of later childhood or adolescent psychological problems. Since it is well established that there is substantial continuity of disorders into adult life (see Section 3), these must be relevant. McLeod et al., in 2007 [322] reviewed published work on the association between parenting and childhood depression, with a meta-analysis of 45 studies. Although the overall results showed poor parenting to be associated with more childhood depression, they also calculated that the effect was quite small ("8\% of the variance"), partly due to inconsistency of methods. The strongest relationship was with parent hostility to the child. Quality and 
style of parenting were also related to early childhood conduct disorders (age 4-5) in wave one of the Longitudinal Study of Australian Children (n 4,936) [323].

Cross-sectional data on several thousand children in Finland and Denmark examined parental violence against their children, which showed a clear dose-response effect on adolescents' mental health problems, both internalising and externalising. There were some differences between sexes and nationalities, but the most surprising and worrying finding was in the frequency of violence in the home - both samples showed around $40 \%$ of adolescents had experienced serious verbal aggression and/or physical violence from their parents during the previous 12 months [324]. Australian twin studies have also showed a strong link between especially harsh punishment of children and later mental illness, independent of any genetic or familial effects [325].

In a recent much simpler and, perhaps, more fundamental study, Oddy et al., in 2010 [326] following through the Western Australian Pregnancy Cohort of 2,900 women for over 14 years, have shown that length of breast feeding predicts problem behaviour and mental health problems throughout childhood, recorded at ages 2, 6, 8, 10 and 14 years. There was a significant difference between those breast feeding for less than or more than six months. Other factors tested did not remove this, but there are still possibilities of confounders not encompassed in the research design.

\subsection{Psychiatric Disorder in Parents}

Parental mental disorder is accepted as an important risk factor for later mental illness in the offspring p146, [327330]. There have been many studies suggesting an increased risk of major depression and anxiety of two or three times, for offspring of parents with serious depression, but very few have been longitudinal studies, and only one has a substantial period of follow-up. From Munich, a four-year follow-up of 2,548 offspring aged 18-24 of patients with depression showed about three times the risk of depression in the offspring, with lesser risks of anxiety and substance abuse, compared with the offspring of non-depressed parents [331333]. This study had strengths in being a large and representative population sample, and making prospective observations, though the follow-up period was only four years.

A recent 20-year follow-up from New York of 101 offspring aged 6-23 years (a mean of 15 years), and 50 controls with non-depressed parents showed higher risks for the research group of about three times for major depression, anxiety disorders, and substance dependence, and also, greater social impairment [334]. Although a very small, clinically selected sample, precluding generalisation, the length of follow-up gives some conviction to these results, confirming, as they do, the general levels of greater risk previously suggested. Interestingly, the offspring have also shown a higher risk of physical illness, especially for cardio-vascular problems, for which they had five times the risk of controls, an issue reviewed in 2003 by Rudisch and Nemeroff [335]. They found depression, especially major depression to be an additional risk factor for both first-event coronary artery disease and further episodes.

The same New York research team have also published early observations of the third generation of these families
[336]. At a mean age of 12 years, and mostly pre-pubertal, nearly $60 \%$ of children with a history of both parental and grandparental depression had shown recognisable psychiatric disorder, mostly anxiety. This was far more than those with a history of parental depression alone. The study is very small, confidence intervals were very wide, and various biases cannot be ruled out, but these results warrant further study. Questions arise as to what degree earlier and effective treatment of depression in either parents or grandparents might modify grandchild risks, and what the effects of early intervention in the grandchildren themselves might affect psychological and social outcomes in adolescence and adulthood for them.

Johnson et al., [316, 337] from the USA study referred to above, suggest that much of the well-established risk for child mental health (and their mental health later as adolescents and adults) attributed to their parents' psychological disturbance may be a product of the inadequate or disturbed parenting associated with that. This hypothesis is somewhat confirmed by further work showing the significance of parenting for a wide range of mental health problems and problem behaviours in the offspring of parents who are psychologically disturbed [338]. The highest risks for aggressive and criminal behaviour in the offspring were for poor maternal control of anger (OR 3.3), lack of maternal affection (2.68), and lack of child supervision (OR 2.24 maternal; 2.23 paternal). A small study of boys in a very criminal culture also concluded that parenting style was crucial [339]. In the Dunedin cohort, childhood-onset delinquency in boys was associated with inadequate parenting, whereas adolescent onset delinquency was not, and the longer term outcome was much worse in the former [153].

This point is more substantially addressed in the British, 'Newcastle 1,000 Families' study from 1947. They were able to demonstrate significant associations of criminal offence rates from childhood to age 33 with ineffective parenting styles. These specifically included poor physical care of children and poor mothering ability, which were probably closely related in particular families. Male offenders in particular were more likely to come from families deemed 'ineffective', slightly more likely to have aggressive fathers. They were less likely to have parents they considered 'effective and kind'. These features were part of a measure of 'deprivation' or 'multiple disadvantage' in childhood which had a big impact on offence rates in both men and women, especially during adolescence [340]. It also had a big impact upon rates of depression by age 33; and in later analyses of a sample of the cohort, poor parenting was associated with major depressive episode especially in women [341].

However, a recent study from the Pittsburgh (USA) Girls Study, with data from 2,451 girls aged 5-8, examined the relationships between child mental health problems and that of their mothers [342]. The most important results indicated that multiple mental health problems in mothers predicted multiple mental health problems in daughters, especially disruptive behaviour and ADHD. Low parental warmth in mothers, and harsh punishment, independently increased the risk of multiple mental health problems in their daughters, but the overall effect of this was small. 
At variance with this is the finding of a large Canadian cohort study of over 4,000 parents and over 6,000 children from 1998 to 2000 [343]. As background to their study they review (with many references) the evidence for the relationship between parent depression and child maladjustment, considering it established beyond doubt (and 2-5 times). Similarly they conclude from previous work that parent depression can affect parenting; that the quality of parenting is related to child emotional, behavioural and social outcomes; and that these various processes inter-relate. In their own follow-up study, three domains of parental behaviour - nurturance, rejection, and monitoring, were found to mediate links between a change in parental depressive symptoms over time and negative child outcomes.

The passing of problems and problem behaviours from parents to children no doubt acts in many ways. Hayatbakhsh et al., [344] in their 3,647 sample followed up to age 21 in Queensland, Australia, showed that it applied to early drug use by children, which was strongly related to parent drug use, but also to poor parental monitoring and supervision and disrupted families. Knappe et al., [345, 346] in their German sample of 1,395 adolescents found that lack of emotional warmth, and dysfunctional family characteristics were associated with higher persistence of social phobia in children, particularly in interaction with parental psycho-pathology. The relationship was especially strong between social phobia in the parents and social phobia in the children, but again, parent over-protection, rejection or lack or emotional warmth were important factors.

Parental 'warmth' was also the outstanding factor in a recent analysis of the long established Whitehall II Study [192]. The study sample was created in 1985-88 from all London-based civil servants aged 35-55 (n 10,308). Data from phases 5 and $7(n=7,276$ and 6,967) related childhood social adversity, social position and adult attachment styles. Low social position (father's occupational social class) was strongly associated with material and emotional adversity, which were also associated with inadequate or insecure attachment styles as adults. In turn these were closely associated with symptoms of depression. But high levels of parental warmth significantly decreased the risk of insecure attachment styles, in the presence of adversities. Adams and Bukowski [283] found that good relationships with parents (and peers) appeared to bring some protection from the negative effects of childhood sexual abuse.

In a recent paper describing a very novel approach to some of these issues, 1,120 children born as a result of IVF treatment ('in-vitro fertilisation') and their parents were examined for depressive symptoms and anti-social behaviour [347]. Because some parents (mother, father, or both) were genetically related to their offspring and some not, the interaction of genetic and environmental factors could be teased out in a new way. They measured depressive symptoms and anti-social behaviour in each parent and in each child, and aspects of parenting behaviour. Both parents provided observations on their children, who were aged 4 to 10 years, a little over half being girls. They particularly differentiated the relationships between mothers and their children and fathers and their children, in genetically related and unrelated groups.
Parent depression and anti-social behaviour were correlated with child depression and anti-social behaviour (except depression in genetically unrelated fathers and children), and parental hostility was generally related both to parent psycho-pathology and to child psycho-pathology, but in such a way as to imply that the genetic relationship was not as important as the environment of the family. Any genetic correlations appeared to be mediated by parenting behaviour. The authors concluded that, from this study, inter-generational transmission of depression would appear to be non-inherited, though where parent hostility was recorded, there appeared to be a relationship between genetic and environmental factors. They also concluded "that there are true environmentally mediated risk effects of negative parenting on children's anti-social behaviour problems." Limitations of the study lay in the exclusive use of postal self-administered questionnaires, and the age of the children. The authors accept that, in the age group 4-10 years, previous research suggests that genetic factors are not prominent in relationships between depression in parents and children, but iare more prominent for adolescents and adults. (see Section 4 above) Perhaps the most interesting finding adds weight to the importance of warmth or hostility in parental attitudes to their children.

There is now evidence that parenting quality and parenting styles are important for later mental health, social behaviour and capacity for relationships, but much more research is needed to tease out the details and gain more precision, to inform preventive action. However, as many of the above authors have commented, evaluated interventions in parenting with very vulnerable groups of parents would seem a sensible if not essential strategy in the light of current knowledge.

\section{Divorce and Separation of Parents; Disrupted \& Dys- Functional Families}

Divorce might appear to be a fairly precise event to record within families of cohort studies. However, it usually leads to single parent status for a time at least, then to remarriage in many, both of which may also affect the child. So its status as a predictor of later mental health problems is not so clear. It is also subject to social change in acceptability, and, indeed, in the status and expectations of marriage; changes in these and related phenomena have been dramatic and profound throughout the western world over the past 50 years.

One element in these many changes is the dramatic reduction in institutional care. It is now relatively uncommon, and so is far less relevant than it was; there is evidence from earlier cohorts that most children placed in effective adoptive families largely recovered from the ill-effects of loss of parents, though there was some indication of difficulties in close relationships. However, those who experienced institutional care throughout childhood and adolescence showed an excess of virtually all types of psycho-social disturbance as adults [348].

For research, even in well conducted, long-term cohort studies, all relevant data have not been collected, because there are serious practical limits on how much can be collected, and because many variables now of lively interest 
were not thought of 30 or 40 years ago. So the collateral characteristics of parents, families, children and communities are available only very partially. And it is necessarily difficult to interpret the significance of divorce and separation within families without knowing about social and economic circumstances, parent personalities, inter-parental conflict, parenting styles and competence, parent drug-use or offending record, re-marriage, new parent characteristics, and so on.

However, in spite of these inevitable inadequacies, evidence has emerged for an important role of divorce or separation in later depression and other psychiatric disorders. In 1991, a meta-analysis by Amato and Keith of over 30 studies identified a significant impact of separation or divorce on educational and occupational achievements, material circumstances in adult life and physical health, as well as psychological well-being. The effects, though significant, were small; the greatest (equivalent to about a third of a standard deviation) was the association with psychological adjustment [349].

What is not so clear is how much this role is dependent upon conflict situations before separation, which has certainly been shown to be important in itself. The importance of the age of the child at the time of separation remains an issue, and how much single parent status and re-marriage affect child mental health outcomes is also still up for debate. This is not surprising, as none of these 'variables' s is homogeneous, however measured, but masks wide variation which will often include both very positive and very negative features.

Divorce or separation is at least an indicator of vulnerability or high risk status for children, and may often be the only point at which intervention is possible. However, intervention in family situations before divorce or separation would be a preferred option where possible, and if suitable interventions are available. Generally speaking, research shows none of the associations of later mental health problems with the death of a parent, but it would surprise no one that children bereaved in a dramatic and terrifying context (such as the bombing of the Twin Towers in New York in 2001) had measurable mental health consequences, especially anxiety disorders, and particularly PTSD [350]. In the Baltimore ECA Study, paternal death increased the risk of major depression, anxiety and panic disorders, but not maternal death, and the authors hypothesise that this could be due to economic consequences for the families [351].

Rodgers, in a 1991 review [126], had noted that broken homes appeared to increase the vulnerability of children to later stresses, associated frequently with mental health and behavioural problems. Weiss et al., in 1999 [236] examined eight relevant studies and found family dysfunction, including early separation to be a risk factor for adult depression in women, but probably correlated with poor parenting and child sexual abuse among other risk factors.

The 1946 British birth cohort showed divorce or separation to be associated with psychiatric disorder at age 26, stomach ulcers and colitis (considered partially psychosomatic disorders) at age 26 , and delinquency by age 21 , but most of the significant associations were for family disruption before the age of five. The exception was psychiatric disorder by age 26 , which had the highest increased risk when parental divorce took place during the subject's adolescence or early adulthood [352]. However, the overall increase in risk related to divorce or separation of parents, when adjusted for social class, birth order and family ties was only x1.6 compared with children not suffering such family disruption.

This was most obvious when the analysis was for any one of the three outcomes (psychiatric disorder, stomach ulcers or colitis, and delinquency). Many early childhood factors showed little effect; those with significant effects on later psychiatric disorder tended to be small groups at the extremes, but most were related to family disruption or parental attitudes and behaviour. Parental divorce or separation had been shown to be most associated with a higher risk of own divorce, with neurosis, and with higher PSE scores at age 26 in women, and delinquency in men [188, 353].

By the age of 43 , the risk of anxiety and depression after parental separation was higher for women than for men, and it was restricted to women who had never married or who had divorced before age 43 [354]. There was no effect for remarriage, and those who were young adults when their parents divorced were also at higher risk of depression. Parental separation was also highly significantly associated with own divorce in men, and both were associated with high PSF scores at 43 [355]. Overall, after follow-up into early midlife, the greatest risk of psychological distress was in women who had experienced both their parents' and their own divorce, and high scores were recorded even before their own divorce [128], suggesting an increased vulnerability.

The same was found also in the 8,000 subjects of the British 1958 cohort - the greatest effect was in women experiencing both parental and their own divorce. For both men and women they found a significant but moderate long-term increase in psychological distress and depression (on the Malaise Inventory) at age 23 and 33 that was associated with parental divorce (ORs <1.6). This was greatest when the child was older, in contrast to the 1946 cohort $[356,357]$. Re-marriage of parents did not change the risks; indeed, restructuring of the family seemed less important than severe disadvantage, and especially experience of being 'in care' (outside the family), but single parent family situation also predicted depression in men at 33 [34]. The association of divorce with depression was still seen at the age of 33 [358]. Death of a parent showed none of these effects in either the 1946 or the 1958 British cohort.

Children often showed signs of distress before parental divorce, and there was evidence that pre-divorce conflict and disruption might be a more important risk factor than divorce itself [356, 358]. Rutter, in 1993 [219] reviewed longitudinal studies and thought there was already substantial evidence that the deleterious effects related to divorce and separation often ante-dated those events; therefore the causative factor was more likely to be the family conflict situation [353, 358]. A review by Birmaher et al., in 1996 [39] of early onset major depressive disorder also found family conflict to be a risk factor. In the Newcastle 1,000 Families cohort, a main predictor of depression in adults up to age 33 was family and parent-marital instability, not always leading to separation or divorce [340]. 
In both New Zealand birth cohorts, there was a measurable association with divorce itself, but in Christchurch, anxiety and depression by age 16 was more closely related to pre-divorce inter-parental conflict $[41,38]$. Suicide attempts were also more in children of divorced parents, but both divorce and prior conflict were part of a melange of disadvantages which, in the most disadvantaged $5 \%$ of children, with very dysfunctional families, was collectively associated with one hundred times the risk of multiple problem behaviours than the most advantaged children [38, 26[. On the other hand, panic attacks and panic disorder, after adjustment for confounders, were not associated with inter-parental violence, though they were with child abuse [277].

Some very interesting data on suicide in young adults arise from the huge cohort (n 610,359) of Norwegian births from 1967 to 1976 , with family data at birth and age 18, and followed through to 2004. Among other precursors of suicide was instability of maternal marital status. Mother being divorced or widowed before the index person was age 18 was associated with an increased risk of suicide, but there was no such risk if the mother was recorded as unmarried both at birth and at age 18 of the index person. It is possible that some of these were cohabiting in a stable relationship but this was not common in the early part of the period. Mother's death increased the risk of suicide only for men. These findings might add weight to the idea that it is marital conflict that underlies the increased risks [117].

Green et al., [194] using the USA National Co-morbidity Survey Replication (NCS-R) found only marginal significance for divorce asssociated with later onset of any DSMIV disorder. But they did find that a group of childhood adversities that together constituted maladaptive family functioning was highly correlated with later psychiatric disorder. The original NCS data, now 20 years old, showed major depression, dysthymia and bipolar disorder related to parental divorce, and that rates of adult illness were increased even more when divorce and reported child abuse occurred together [284].

Recent analyses from the Christchurch cohort have shown a progressive but rather weakly significant association of anxiety (but not depression, suicidal behaviour or substance dependence) between ages 21 and 25 with experience of single parenthood before age 16. This was weakened further when controlling for a series of disadvantages correlated with single parenthood. They included lower maternal age, lower levels of parental education, poorer socioeconomic status, more family problems, exposure to childhood sexual and physical abuse, parental illicit drug use and criminal offending, and lower IQ. However, as the authors admit, it is almost certain that some of these factors are consequences of being a single parent, at least in some cases, so there may be serious over-control of the analysis [359]. Single parenthood must relate to divorce in a complex and very variable way, but it is not equivalent in any way.

In a large US study focussed on alcohol problems over 43,000 adults were interviewed in 2001-2002. Among other things, childhood or adolescent parental divorce or separation appeared to be a strong predictor of offspring life-time alcohol dependence [360].
In the Northern Finland cohort, both treated alcoholism and drunken driving offences in adolescents were strongly associated with parental divorce or separation and single parent status [361]. In men violent criminal offences (but not non-violent offences), were also associated with divorce and separation of parents, being part of a spectrum of characteristics related particularly to single parent family status [362, 363]. Parent child-rearing issues were implicated, and psychological disturbance was also part of the outcome for these adolescent offspring. Similarly, in the Finnish 1981 birth cohort of over 5,000 subjects, 'any psychiatric disorder' at age 18 , but particularly depression, were strongly predicted by not living with two biological parents at age eight [42]. The OR for 'any disorder' was 2.2; for depression it was 2.3; and for psychosis 2.5; but anti-social personality disorder and substance abuse gave ORs of 3.5. Mother-alone and mother-absent families in the rather special AfricanAmerican community of Woodlawn, Chicago, were associated with later suicidal behaviours by the age of 32 [198]. But it has to be questioned as to how much social statistics from such disparate communities can be compared or contribute towards any one conclusion.

In Tampere, Finland, 1,656 16-year old adolescents were followed up to age $22.24 \%$ of them had experienced parental divorce before the age of 16 and for these, depression was significantly more common in both males and females, as was low self-esteem at age 16. In girls only, closeness to the father appeared to be protective of depression after divorce [124].

In a cohort study in North-Eastern USA following 354 children aged 5 into their 20s, family violence was the strongest predictor of adult major depression in young adults, diagnosed in nearly one quarter of the whole sample [31]. In over 1,000 children followed through from before birth into their 30s in Providence, Rhode Island, USA, family disruption, largely involving separation or divorce, was associated with about two times the rate of depression in adult life, especially for depression of onset before age 14 . Inter-parental conflict was independently associated with depression, and both together increased the risks even further $[364,365]$.

In a similar design, following up over 4,000 children from before birth to age 14, the Queensland, Australia cohort also found a small but significant excess of serious anxiety and depression even at 14 , in the group of children exposed to divorce or separation, though not for single parent status if retained for the first five years. Data on later years have not been presented. Repeated parent-partner problems within the family was additive [366].

Separation of children not implying family conflict or break-up of relationships has not featured in this research much, but an interesting paper from Finland looked at depression in adults around age 60 (two measures two years apart) who were part of a 1934-44 birth cohort (n 1,658). The years of the second world war offered an un-planned experiment as many children were separated from fathers in the army and many others were separated from both parents by unaccompanied evacuation [367]. Former evacuees (n 410) revealed $20 \%$ more severe depressive symptoms (Beck) than non-evacuees, and even for mild symptoms there was 
an odds ratio of 1.7 (CI 1.1-2.6) but those separated from their military father (n 744) showed no differences from nonseparated peers. Those evacuated as toddlers showed the least effects, but those evacuated as infants showed $23 \%$ more severe depression, and as school children $30 \%$ more.

There is no reason, without careful analysis, to think that evacuation experiences were closely similar in different countries during the war. A study of evacuees in Britain used a sample of 870 people aged 62-72 years. Those evacuated when aged 4-6 years, or who received poor foster care had increased risks of depression and anxiety, and high levels of self-criticism. Those evacuated at age 13-15, who received good care, had lowered risks. The quality of foster-home nurture was an important factor for all [368].

Few studies even among the cohorts have had opportunity to study the effect on children of abused parents. A paper from the ALSPAC team in Bristol UK, whose cohort started during pregnancy, has been able to explore the inter-generational effects up to age 7 [258]. 13,971 women enrolled in the ALSPAC cohort between April 1991 and December 1992 gave birth to a child who survived infancy. Data were collected before birth and at ages 4 and 7 (see [255] and Section 10). $17.1 \%$ of women had experienced abuse in their own childhood, and increased offspring adjustment problems were associated at age 4 and 7 with all forms of abuse, with the rated severity of abuse (mostly), and with multiple types of abuse. These associations were consistent for data from both parent and teacher ratings of child adjustment.

It was also evident that children of abused mothers had an increased risk of experiencing a wide range of adversity, increasing with the severity of the maternal abuse. These included being physically hurt, and parental separation, but not death. The results overall suggested a cumulative impact of maternal abuse experience, family adversity, and negative events as children grow older so that these children had very poor adjustment trajectories between age 4 and 7 . It will be interesting (and hopefully very instructive) to follow through these children into older ages, but interventions cannot be eschewed if clinically indicated.

\section{Summary of Results}

\subsection{Summaries According to Sections 3-12}

As Fergussen and Horwood wrote in 2005 (143): "... these disorders are multi-causally determined and reflect the aggregate effects of social, family (including genetic), individual, peer and other factors."

\subsubsection{Psychological Disturbance in Childhood and Ado- lescence. (Section 3)}

This is the most well-established precursor, though not a cause of adult psychiatric disorder. There is strong evidence of continuity of morbidity; that is, much mental illness in adults starts in childhood or adolescence, and some shows a continuity of morbid symptoms from early childhood through adolescence into adult life. This applies particularly to the common mental disorders of anxiety and depression, but also to serious depressive illness These children can be identified but very often are not. The increased risk of adult persistent depression in adolescents with persistent depres- sion may be over ten times. Apart from other factors, psychotic and pre-psychotic symptoms do occur in children as well as adolescents and are strongly indicative of later nonaffective psychosis. In the first place, children's mental health problems should be identified and treated appropriately for their own sake, but also because they represent a high risk for adult illness. This needs wider recognition, and more and better trained professionals.

\subsubsection{Genetic Influences. (Section 4)}

There are undoubted genetic components in the causation of psychoses, especially of schizophreniform disorders, and heritable risks in both schizophrenia and bipolar disorder. The life-time risk of psychosis with one parent with schizophrenia is probably around $10 \%$, compared with $1 \%$ for the general population. The contribution may be perceived largely as conferring increased vulnerability or resilience to stressors throughout life. In some cases the increased susceptibility may reside in CNS disorders. Condition at birth and neurological deficits in childhood may be indicators of genetic predisposition. At least 20 genes or gene variants have now been associated specifically with schizophrenia, but genetic intervention is not yet in sight. The dominant hypothesis is now that schizophrenia is at root a neurological disorder, predisposing to illness in response to later stimuli.

\subsubsection{Neurological Deviance in Childhood; Intelligence, Brain Damage and Disorder. (Section 5)}

There is very strong evidence for demonstrable brain damage or disorder, present at birth or incurred during birth, being associated with a greatly increased risk of psychosis, especially schizophrenic spectrum disorders, possibly as much as five times, and is likely to be a prime cause. Damage may arise also in CNS infections. There are many indicators of neurological deviance which are susceptible to identification in childhood, including birth weight, delayed milestones and measured intelligence. Evidence of association with anxiety and depression is not very strong.

\subsubsection{Features of Personalitv. (Section 6)}

Neuroticism, measured using standard instruments, has been commonly found as a precursor of adult psychiatric symptoms and disorder, but in a very non-specific way. It may be an indicator of vulnerability and poor coping skills, or represent early manifestations of persistent disorder. More recently 'locus of control' and measures of self-esteem have been shown to be useful indicators of future psychological health, and recording positive experiences and events in childhood has shown them to be progressively associated with good mental health as adults.

\subsubsection{Behaviour in Childhood and Adolescence. (Section 7)}

Measurement of behaviour is difficult and can be dimensional or categorical, with different implications for research. Both generalised aspects of difficult behaviour and specific diagnoses such as conduct disorders and ADHD can be related to later mental illness. But they have themselves causal factors and processes and cannot readily be separated from other factors either conceptually or operationally. In some cases, behavioural disturbance is related to neurological defects or incipient schizoid traits. Certain observed behav- 
iours, especially inattention and hyperactivity, withdrawn, deviant, aggressive, anti-social and disruptive behaviour, consistently show associations with later depression and anxiety, as well as delinquent and criminal behaviour.

Bullying is a specific aggressive behaviour linked to later psychiatric disorder, but the most disturbed are young men who are both bullies and bullied. All these disorders have many causes reflecting genetic, family, social, individual, peer and other factors. Abnormal behaviour may also provoke more negative life events than other children experience, and these are also associated with later anxiety and depression. Alcohol and other drug abuse is, also related, not surprisingly; and there is now a body of evidence specifically linking cannabis use in childhood and adolescence with later psychosis, perhaps doubling the risk, but the interpretation is disputed.

\subsubsection{Poor School Performance; Poor Educational Achievement. (Section 8)}

Poor school performance and low levels of educational achievement by the end of formal schooling are known to be associated with adult anxiety and depression, with an increased risk of probably two or three times, and this is confirmed by cohort studies. Difficult and deviant behaviour, and neurological deficits of any type in childhood are likely to have an impact on school performance and achievements, and these may mediate a higher risk. This is likely to explain associations with schizophrenia.

It is unlikely to represent a primary causal factor, representing more often a late- childhood manifestation of a preexisting relatively high risk, but it may well be a contributory cause of later mental health problems (as with delinquent and criminal behaviour, especially illiteracy) and it does provide a relatively easily identifiable group of high risk adolescents for preventive intervention.

\subsubsection{Childhood Adversity; Life Events; Multiple} Disadvantage. (Section 9)

Measurement of adversities in childhood have most commonly had to repy upon retrospective data, but recent research has suggested that they are sufficiently reliable to be very useful. Adverse situations and negative life events include very many different types of experience, provoking many individual responses, but relatively few have been measured and studied. Generalisation is, therefore, inescapable, but so much research suggests very non-specific relationships between a wide range of childhood adversities and a wide range of adult psychological problems or mental illness including suicide, that this may prove to be not so important an issue. It is also obvious that many adversities, however measured, are related to each other, and tend to cluster. However, it is possible to say that a wide range of adverse experiences in childhood, in which we can include negative life events and chronic ill-health, have been shown to increase the risk of psychiatric disorder in adulthood. Studies tend to find the increased risk for a wide variety of factors to be around two to three times. There are inconsistent results for differences between boys and girls.

More importantly, it is commonly found that children experiencing multiple adversities of many types have an in- creased risk of around three times, for anxiety and depression, suicidal behaviour and admission to hospital for serious psychiatric disorder. The more recorded adversities, the greater the risk of psychological ill-health later. In one reliable study reporting in this way, the $5 \%$ most disadvantaged children had an increased risk of 100 times compared with the $50 \%$ least disadvantaged children.

It does not require proof of adult mental illness to justify interventions to reduce childhood adversity, poverty, and family dysfunction, but there is evidence that reducing gross and multiple disadvantage will reduce later psychological distress and psychiatric disorder.

\subsubsection{Child Abuse, Neglect and Mal-Treatment. (Section 10)}

Substantial proportions of children and young people are apparently subjected to serious verbal, physical and sexual abuse, severe mal-treatment and neglect in every community where it has been investigated. But also many children are resilient and, achieve healthy, adjusted adult lives even even after child abuse, dependent also upon the type, degree and circumstances of abuse.

Child abuse is difficult to measure and study and definitions do not easily achieve consistency. Some reviews have concluded that child physical abuse has been experienced by $10-31 \%$ of men and $6-40 \%$ of women; and child sexual abuse by $3-29 \%$ of men and $7-36 \%$ of women, but these wide ranges are not very helpful. A large UK study interviewing young adults gave: serious mal-treatment $16 \%$ : contact sexual abuse $11 \%$ (16\% women and 7\% men); physical abuse $7 \%$ (12\% women and $17 \%$ men); emotional abuse $6 \%$; absence of care $6 \%$; absence of supervision $5 \%$. Replication of this some years later gave largely similar results.

It is not surprising that serious abuse is associated with later serious psychiatric disorder, as well as personality disorders, self-destructive and violent behaviour, physical illness, teenage pregnancy, and problems raising their own children.

Multiple effects and general psycho-pathology commonly reveal non-specific relationships with almost all psychiatric diagnostic categories. If outcomes are varied and non-specific, so, it seems, are the putative causes. No one type of child abuse, however defined, stands alone, but should be seen in the context of family values, behaviour, conflict, socio-economic circumstances, parental illness, and so on, all of which may also change throughout the period of childhood.

Four principle risk factors in parents have emerged: young parental age; low educational achievements; adverse childhood experiences, particularly parental exposure to abuse or neglect in their own childhood; and parental psychiatric history, including a history of alcohol or drug abuse.

Depressive and anxiety disorders in adulthood, including PTSD, are consistently shown to be increased following all types of child abuse, with some gradation with the seriously of the type of abuse, frequency, timing and relationship with the abuser. The effects are seen at least into middle age in the longest cohorts. Suicide, ideation and attempts are also increased. 
Evidence for psychosis or psychotic symptoms is rather varied and inconsistent but less so for serious sexual abuse. It may be that other factors are involved in the relationships where they emerge but it seems not to be a simple relationship.

Treatment is possible but not sufficiently available, cognitive behaviour therapy (CBT) being generally favoured, but prevention should be a major focus. It is far from easy, and, unfortunately, there are not many ideas in evidence. It needs politicians as well as professionals to be engaged with this issue if present and future generations of children are to be adequately protected.

\subsubsection{Parenting, Parent-Child Relationships and Parent Psychological Disturbance. (Section 11)}

Parenting style and quality has only recently been subject to much study, and measures are limited, but the literature is growing. Many studies focus on degrees of care and control by parents, data being mostly retrospective from the children as adolescents or adults. Parent-child interactions characterised by affective enhancement (affectional bonds) and low control (encouragement of autonomy) appeared protective of psychiatric symptoms in adulthood, whereas relationships characterised by affective impoverishment (neglectful relationships) and over-protection (intrusive behaviour) were apparent risk factors. For

depression and anxiety in adults, the increased risk may be between 1.5 and 3 times. And the evidence also links parenting style and quality with social behaviour and capacity for relationships, including capacity for parenting their own children. Parents own experience of childhood abuse and other adversities may be important in mediating poor parenting, Parents who suffer from psychiatric disorders pose higher risks for the mental health of their children, and this may be through poor parenting. Most commonly in the research reviewed here, fathers are at least as important in determining good or bad outcomes for their children as mothers. Much more research is needed to tease out the details if preventive action is to be undertaken, but intervention in parenting with very vulnerable groups of parents would seem a sensible and important strategy, even in the light of current knowledge. There are already evaluated intervention studies.

13.1.10. Divorce and Separation of Parents; Disrupted and Dysfunctional Families. (Section 12)

Divorce cannot stand alone as a factor affecting children. It is often preceded by separation, will often be the culmination of inter-parental conflict, and may result in a nonoptimal family situation for the child. Divorce or separation of parents usually emerges strongly as a factor in all periods of childhood and adolescence, associated with later anxiety and depression anti-social behaviour and other outcomes, but evidence suggests that prior inter-parental conflict may be a more important determinant. The effect may not be great; the evidence from the large cohorts suggests an increased risk of depression in early adult life of somewhat less or somewhat more than $\mathrm{x} 2$, but it is usually found to be greater in women, and is increased by own divorce as an adult. Generally speaking, research shows none of these associations with the death of a parent unless in very dramatic circumstances.
Separation of children from one parent without conflict (as fathers going away as soldiers) appears to have no bad effects. The mental health of children evacuated during wars and fostered appears to depend largely upon the quality of the foster-family. More related studies would be welcome.

Dysfunctional families, including inter-parental violence, do create greater risks of adult anxiety and depression and probably suicide, There is evidence of inter-generational effects, with the offspring of parents who experienced abuse in their own childhood experiencing more adversities, negative events and adjustment problems, but this research is not yet very extensive.

Divorce or separation is at least an indicator of vulnerability or high risk status for children, and may be a key point at which intervention is possible. However, intervention in family conflict situations before divorce or separation would be a preferred option where possible, and if suitable interventions are available.

\subsection{Conclusions Concerning Research}

1. In spite of many methodological difficulties, longitudinal studies continue to provide a great deal of extremely valuable evidence relating childhood factors to adult psychiatric disorder, and their potential for more remains, if they can continue to be funded.

2. However, given the variety of studies, methods and populations, the evidence for generalisable associations inevitably remains incomplete, and conclusions are always tentative and provisional, more for some variables than others.

3. Probably the most clearly established association is between mental health problems / psychological disturbance in childhood and adolescence, and mental health problems / psychiatric disorder in adulthood, and it seems likely that the key interpretation is that of continuity of morbidity. This provides an opportunity for early intervention by identifying and treating childhood problems seriously and thoroughly, but evaluation of interventions is required.

4. There seems little doubt that there are significant heritable genetic contributions to psychosis, but usually requiring later factors for expression. There are probably also genetic contributions to vulnerability and resilience which affect later ability to resist and cope with stress, but more research will be required to elucidate this. Similarly, brain damage or disorder, before during or after birth increase the risks of recurrent psychiatric disorder which also may represent an increased vulnerability, requiring additional factors to cause serious problems; these factors also need elucidating.

5. Personality features, especially neuroticism, deviant child behaviour, and poor school performance appear to be important indicators of children vulnerable to stress or already revealing precursors of psychiatric disorder, in many cases related to genetic factors or CNS damage. We need to evaluate ways of identifying at-risk children so that preventive measures may most effectively and efficiently be implemented.

6. There is clear evidence that serious childhood adversity increases the risk of recurrent psychiatric disorder 
throughout life. The most serious is child abuse, especially child sexual abuse, which the evidence suggests is surprisingly and disturbingly common. Depressive and anxiety disorders are most prominent but most diagnostic categories tend to show excesses. Parental conflict leading to divorce or separation appears to be an important causal factor. Poor parenting must now be considered demonstrably important as a cause of later mental health problems, but is difficult to isolate from other intra-family adversities.

There has been a great increase in research related to child abuse in recent years and prevention of child abuse should be a high priority with the evidence available now. Further careful research, especially in large population cohorts and samples will add further weight to that evidence, but perhaps most needed is further understanding of the causal factors and processes in families and communities that lead to child abuse, and then to a life-time of mental distress. Parenting education and training may be a crucial element; interest in evaluating its effectiveness need to be strongly encouraged (eg. [369].

7. Multiple adversities and multiple experiences of abuse have strong evidence as causing many very serious social and psycho-social consequences affecting individuals, families and communities, and must be considered among the most important potentially preventable 'causes' of psychiatric disorder throughout life. It is, of course, not a single 'cause' but an accumulation of several or many 'causes', which appear to be cumulative in their ultimate effects on children, and families with the highest levels of multiple adversity should be a very high priority for early identification and relief. Further work should aim to render such identification effective and efficient in bringing help to children most in need. Identification should be possible within school systems or their associated services, and these should offer opportunities for evaluation.

\subsection{The Potential for Prevention}

No review of existing evidence in a field as complex as the causes of mental illness can be anything near complete. There are too many facets, too many interacting, overlapping factors involved, some of which are barely touched by research, some with a considerable body of research, and few with unarguable conclusions. And those features of childhood with the most research attention, or the best evidence for their contribution to mental illness, are not necessarily the most important. Nor are they necessarily the most practicable to prevent.

The main focus of this research review was evidence for situations, events or characteristics of childhood which were potentially ameliorable. Rapidly increasing knowledge of genetics may create the potential for gene modification, but this lies in the future.

Preventing brain damage before, during or after birth is already a very high priority in any developed obstetric and child health service, and many successes have been recorded. It is, however, a continuing challenge, particularly as less and less mature babies are saved, alive but with greatly increased risks of damage. Drug and alcohol abuse in pregnancy also offer increasing risks for babies in many societies. We cannot be so sure of the influence of ante-natal and post-natal maternal mental health on the new-born child, but it is likely to be important for the child's continuing emotional and social development subsequently. We are making progress towards improving maternal mental health, important for itself but also for the child and the family [370]. Prevention is surely not beyond our imagination, but the challenge is far more to politicians and social agencies than medicine, and current successes are limited.

Although many disabled children come through their childhood without serious psychological problems, any physical damage in a child is an important risk factor for psychological distress and mental ill-health, both in childhood and later. This may be especially true of damage to the central nervous system, which may not be evident to most lay observers, including family, friends, and teachers at school, but may produce abnormal behaviour which is obvious to everyone. Such situations need very thorough specialist investigation, treatment where possible, and full consultation, information and support for the child, parents and others involved, if additional psychological damage is to be minimised.

This shows the importance of taking behavioural problems in children seriously. Hopefully, children with neurological deficits will be identified early in life, but appropriate and thorough investigation of behaviour problems should be the norm whatever the age of the child. Children may demand attention largely by reason of unwanted behaviours for which there is no neurological explanation. These will include those who show worrying features of personality; those who show school failure, especially where inconsistent with expectations; and those who show psychological disturbance.

Investigation, of course, may show nothing to explain the behaviour, in which case only the behaviour itself can be addressed. Behaviours of particular concern include inattention and hyper-activity, withdrawn, deviant, aggressive, antisocial and disruptive behaviour, delinquent and criminal behaviour, and abuse of alcohol and other drugs. Cognitive behaviour therapy is currently considered the treatment approach most likely to succeed, but professional assessment and prescription is essential.

Where underlying or surrounding factors are discovered which go some way at least to explaining the behaviour, these must be addressed, and in many cases this will need assisting the family as a whole. But psychological disturbance is frequently missed in children, not least depression, and we might achieve substantial benefits in terms of adult mental illness by treating it in children adequately, though of course, it justifies treatment for itself.

All this is well known and, to a limited extent, common practice, though most service systems are grossly inadequate to serve the needs of all the children and families in their communities. However, addressing underlying causes of behaviour and psychological disturbance in children when they lie in parental behaviour or family circumstance is often not addressed. Child abuse is certainly not exclusively parental, but is most commonly parental, and there are few programmes for intervention other than removing the child, and, it seems, few ideas for how to prevent the abuse happening or continuing. 
The evidence from the literature suggest that child abuse is a very big problem in most societies, and one which is frequently ignored. We need a very aggressive programme to address this if large numbers of our children are going to be protected. The first need is for thorough evaluation of any existing programmes which might be considered preventative of child abuse. However, these are limited in scope, and evaluation might take a long time. New strategies would also be welcome, and a commitment from professionals and politicians to address this issue. It is possible that productive ideas will come not only from professional, academic and research sources but from those who are the victims of abuse and suffer the greatest consequences. "Stuart, a Life Backwards" by Alexander Masters should be read by all concerned [310].

There is, perhaps, a continuum from serious and serial child sexual abuse to poor parenting skills, but interventions can only be prescribed when the situation has been analysed and specific issues have been identified. A need is increasingly recognised for parenting education and training for inadequate or unusually stressed parents, and there are now examples of evaluated programmes. Identifying at-risk parents is clearly one issue; interference in families is another, but where children are at risk, their welfare must be given priority, and this is likely also to benefit parents once help is accepted.

Some easily recognised situations place children at an increased risk and should be used to offer help; an obvious example is separation or divorce of parents, an opportunity to take preventive action on behalf of the child. But though there is evidence of the higher risk of mental illness in children of divorced parents, there is more evidence that this may be commonly the result of parental and family conflict before separation or divorce renders it obvious. It is much more difficult to conceive of a preventive strategy for this, except through identifying early signs of problematic child - or parental - behaviour, and intervening, also far from easy [369].

When parents suffer from recognised mental illness and have sought psychiatric treatment, the context of their identification may make it a little easier to get agreement for intervention on behalf of the children. Quite substantial family programmes are now being implemented in some countries; a majority of health districts in Finland have trained psychiatric service professionals in the "Effective Family Programme", for which early evaluation is hopeful. It is intended to extend it to primary health care [371, 372]. Older programmes in the USA and elsewhere have been evaluated $[369,373]$.

Sometimes the style and quality of parenting may appear to stand alone as an issue, though more commonly it will be associated with other problems in the parents or the family situation. So parenting education may be applicable to many situations, and there is now evidence of effective programmes (eg. [369, 374]. Rarely is real interest shown by governments, unless perhaps with regard to anti-social behaviour, but there needs to be concern for the real challenges to parents of the ever-changing social context in which they must bring up children. This may apply particularly to the single parents now so common in Western societies. Parent- ing quality and parent training are issues which are not going to go away, and we need to address them much more comprehensively.

The main interest of this project was the potential for intervention in children and in families to prevent potentially harmful situations and events from happening in the first place, or to ameliorate deleterious effects by early intervention. There is much evidence of potentially preventable factors, though we would like more, and evidence building of the effectiveness of interventions. The potential is there, but it will need more political will and professional commitment if it is to be realised to any substantial degree.

\section{CONFLICT OF INTEREST}

The authors confirm that this article content has no conflicts of interest.

\section{ACKNOWLEDGEMENT}

Declared none.

\section{REFERENCES}

[1] Lavikainen J, Fryers T, Lehtinen V. Improving Mental Health Information in Europe. $1^{\text {st }}$ ed. Helsinki: STAKES 2006.

[2] Fryers T, Melzer D, Jenkins R. Social inequalities and the common mental disorders: a systematic review of the evidence. Soc Psychiatry Psychiatr Epidemiol 2003; 38(5): 229-37.

[3] Melzer D, Fryers T, Jenkins R. Social inequalities and the distribution of the common mental disorders. $1^{\text {st }}$ ed. Hove; New York: Psychology Press 2004.

[4] Waterston T, Alperstein G, Stewart BS. Social capital: a key factor in child health inequalities. Arch Dis Child 2004; 89(5): 456-9.

[5] Goldberg D, Huxley P. Common mental disorders. $2^{\text {nd }}$ ed. London; New York: Routledge 1992.

[6] Barlow J, Coren E, Stewart-Brown S. Meta-analysis of the effectiveness of parenting programmes in improving maternal psychosocial health. Br J Gen Pract 2002; 52(476): 223-33.

[7] Morgan C, Fisher H. Environment and Schizophrenia: environmental factors in schizophrenia: childhood trauma, a critical review. Schizophr Bull 2007; 33(1): 3-10.

[8] Bendall S, Jackson HJ. Childhood trauma and psychotic disorders: a systematic, critical review of the evidence. Schizophr Bull 2008; 34(3), 568-79.

[9] Skodol AE, Bender DS, Pagano ME, et al. Positive childhood experiences: resilience and recovery from personality disorder in early adulthood. J Clin Psychiatry 2007; 68(7): 1102-8.

[10] Marmot MG. Early life and adult disorder: Research themes. $\mathrm{Br}$ Med Bull 1997; 53(1): 3-9.

[11] Reef J, Diamantopoulou S, van MI, Verhulst FC, van der EJ. Developmental trajectories of child to adolescent externalizing behavior and adult DSM-IV disorder: results of a 24-year longitudinal study. Soc Psychiatry Psychiatr Epidemiol 2011; 46(12): 1233-41.

[12] Reef J, Diamantopoulou S, van MI, Verhulst F, van der EJ. Child to adult continuities of psychopathology: a 24-year follow-up. Acta Psychiatr Scand 2009; 120(3): 230-8.

[13] Reef J, van MI, Verhulst FC, van der EJ. Children's problems predict adults' DSM-IV disorders across 24 years. J Am Acad Child Adolesc Psychiatry 2010; 49(11): 1117-24.

[14] Reef J, Diamantopoulou S, van MI, Verhulst F, van der EJ. Predicting adult emotional and behavioral problems from externalizing problem trajectories in a 24-year longitudinal study. Eur Child Adolesc Psychiatry 2010; 19(7): 577-85.

[15] Dekker MC, Ferdinand RF, van Lang ND, Bongers IL, van der EJ, Verhulst FC. Developmental trajectories of depressive symptoms from early childhood to late adolescence: gender differences and adult outcome. J Child Psychol Psychiatry 2007; 48(7): 657-66.

[16] Fergusson DM, Woodward LJ. Mental health, educational, and social role outcomes of adolescents with depression. Arch Gen Psychiatry 2002; 59(3): 225-31. 
[17] Roza SJ, Hofstra MB, van der Ende J, Verhulst FC. Stable prediction of mood and anxiety disorders based on behavioral and emotional problems in childhood: a 14-year follow-up during childhood, adolescence, and young adulthood. Am J Psychiatry 2003; 160(12): 2116-21.

[18] Kandel D, Davies M. Adult sequellae of adolescent depressive symptoms. Arch Gen Psychiatry 1986; 43: 255-62.

[19] Wadsworth ME, Rodgers B. Longterm follow-up studies; a critical overview. [Review] [203 refs]. Rev Epidemiol Sante Publique 1989; 37(5-6): 533-47.

[20] Bardone A, Moffitt TE, Caspi A, Dickson N, Silva PA. Adult mental health and social outcomes of adolescent girls with depression and conduct disorder. Dev Psychopathol 1996; 8: 811-29.

[21] Lewinsohn P, Rohde P, Klein D, Seeley J. Natural course of adolescent major depressive disorder,1; continuity into young adulthood. J Am Acad Child Adolesc Psychiatry 1999; 38: 56-63.

[22] Rao U, Hammen C, Daley S. Continuity of depression during the transition to adulthood: a 5-year longitudinal study of young women. J Am Acad Child Adolesc Psychiatry 1999; 38: 908-15.

[23] Hofstra MB, van der Ende J, Verhulst FC. Child and adolescent problems predict DSM-IV disorders in adulthood: A 14-year follow-up of a Dutch epidemiological sample. J Am Acad Child Adolesc Psychiatry 2002; 41(2): 182-9.

[24] Weissman MM, Wolk S, Wickramaratne P, et al. Children with pre-pubertal-onset major depressive disorder and anxiety, grown up. Arch Gen Psychiatry 1999; 56: 794-801.

[25] Wals M, Verhulst F. Child and adolescent antecedents of adult mood disorders. Curr Opin Psychiatry 2005; 18(1): 15.

[26] Fergusson DM, Horwood LJ. The Christchurch Health and Development Study: review of findings on child and adolescent mental health. Aust NZ J Psychiatry 2001; 35(3): 287-96.

[27] Fombonne E, Wostear G, Cooper V, Harrington R, Rutter M. The Maudsley long-term follow-up of child and adolescent depression. 1. Psychiatric outcomes in adulthood. Br J Psychiatry 2001; 179: 210-7.

[28] Fombonne E, Wostear G, Cooper V, Harrington R, Rutter M. The Maudsley long-term follow-up of child and adolescent depression. 2. Suicidality, criminality and social dysfunction in adulthood. Br J Psychiatry 2001; 179: 218-23.

[29] Kim-Cohen J, Caspi A, Moffitt Tea. Prior juvenile diagnoses in adults with mental disorder; developmental follow-back of a prospective-longitudinal cohort. Arch Gen Psychiatry 2003; 60: 70917.

[30] Gyllenberg D, Sourander A, Niemela S, et al. Childhood predictors of later psychiatric hospital treatment: findings from the Finnish 1981 birth cohort study. Eur Child Adolesc Psychiatry 2010; 19(11): 823-33.

[31] Reinherz HZ, Paradis AD, Giaconia RM, Stashwick CK, Fitzmaurice G. Childhood and adolescent predictors of major depression in the transition to adulthood. Am J Psychiatry 2003; 160(12): 2141-7.

[32] Hofstra MB, van der Ende J, Verhulst FC. Adolescents' selfreported problems as predictors of psychopathology in adulthood: 10-year follow-up study. Br J Psychiatry 2001; 179: 203-9.

[33] Van Os J, Jones P, Lewis G, Wadsworth M, Murray R. Developmental precursors of affective illness in a general population birth cohort. Arch Gen Psychiatry 1997; 54(7): 625-31.

[34] Buchanan A, Ten Brinke J, Flouri E. Parental background, social disadvantage, public "care," and psychological problems in adolescence and adulthood. J Am Acad Child Adolesc Psychiatry 2000; 39(11): 1415-23.

[35] Clark C, Rodgers B, Caldwell T, Power C, Stansfeld S. Childhood and adulthood psychological ill health as predictors of midlife affective and anxiety disorders: the 1958 British Birth Cohort. Arch Gen Psychiatry 2007; 64(6): 668-78.

[36] Copeland WE, Shanahan L, Costello EJ, Angold A. Childhood and adolescent psychiatric disorders as predictors of young adult disorders. Arch Gen Psychiatry 2009; 66(7): 764-72.

[37] Beautrais AL. Risk factors for suicide and attempted suicide among young people. Aust NZ J Psychiatry 2000; 34(3): 420-36.

[38] Fergusson DM, Woodward LJ, Horwood LJ. Risk factors and life processes associated with the onset of suicidal behaviour during adolescence and early adulthood. Psychol Med 2000; 30(1): 23-39.

[39] Birmaher B, Ryan ND, Williamson DE, et al. Childhood and adolescent depression: A review of the past 10 years, Part I. J Am Acad Child Adolesc Psychiatry 1996; 35(11): 1427-39.
[40] Patton GC, Coffey C, Posterino M, Carlin JB, Bowes G. Life events and early onset depression: cause or consequence? Psychol Med 2003; 33(7): 1203-10

[41] Feehan M, McGee R, Williams SM, Nada-Raja S. Models of adolescent psychopathology: Childhood risk and the transition to adulthood. J Am Acad Child Adolesc Psychiatry 1995; 34(5): 670-

[42] Sourander A, Multimaki P, Nikolakaros G, et al. Childhood predictors of psychiatric disorders among boys: A prospective community-based follow-up study from age 8 years to early adulthood. J Am Acad Child Adolesc Psychiatry 2005; 44(8): 756-67.

[43] Sourander A, Haavisto A, Ronning JA, et al. Recognition of psychiatric disorders, and self-perceived problems. A follow-up study from age 8 to age 18. J Child Psychol Psychiatry 2005; 46(10): 1124

[44] Ford T, Collishaw S, Meltzer H, Goodman R. A prospective study of childhood psychopathology: independent predictors of change over three years. Soc Psychiatry Psychiatr Epidemiol 2007; 42(12): 953-61.

[45] Najman JM, Hayatbakhsh MR, Clavarino A, Bor W, O'Callaghan MJ, Williams GM. Family poverty over the early life course and recurrent adolescent and young adult anxiety and depression: a longitudinal study. Am J Public Health 2010; 100(9): 1719-23.

[46] Biederman J, Petty CR, Faraone SV, et al. Antecedents to panic disorder in nonreferred adults. J Clin Psychiatry 2006; 67(8): 117986.

[47] Cannon M, Caspi A, Moffitt TE, et al. Evidence for earlychildhood, pan-developmental impairment specific to schizophreniform disorder: results from a longitudinal birth cohort.[see comment]. Arch Gen Psychiatry 2002; 59(5): 449-56.

[48] Welham J, Scott J, Williams GM, et al. The antecedents of nonaffective psychosis in a birth-cohort, with a focus on measures related to cognitive ability, attentional dysfunction and speech problems. Acta Psychiatr Scand 2010; 121(4): 273-9.

[49] Scott J, Martin G, Welham J, et al. Psychopathology during childhood and adolescence predicts delusional-like experiences in adults: a 21-year birth cohort study. Am J Psychiatry 2009; 166(5): 567-74.

[50] Post RM, Leverich GS, Kupka RW, et al. Early-onset bipolar disorder and treatment delay are risk factors for poor outcome in adulthood. J Clin Psychiatry 2010; 71(7): 864-72.

[51] Colman I, Jones PB. Birth cohort studies in psychiatry: beginning at the beginning. Psychol Med 2004; 34: 1375-83

[52] Kendler KS, Aggen SH, Knudsen GP, Røysamb E, Neale MC, Reichborn-Kjennerud $T$. The structure of genetic and environmental risk factors for syndromal and subsyndromal common dsmiv axis $\mathrm{i}$ and all axis ii disorders. Am J Psychiatry 2011; 168: 2939.

[53] DelBello MP, Geller B. Review of studies of child and adolescent offspring of bipolar parents. Bipolar Disord 2001; 3(6): 325-34.

[54] Ripke S, Sanders AR, Kendler KS, et al. Genome-wide association study identifies five new schizophrenia loci. Nat Genet 2011; 43(10): 969-76.

[55] Hankin BL, Abramson LY. Development of gender differences in depression: Description and possible explanations. Ann Med 1999; 31(6): 372-9.

[56] McGuffin P, Cohen S, Knight J. Homing in on depression genes. Am J Psychiatry 2007; 164(2): 195-7.

[57] Wiles NJ, Peters TJ, Leon DA, Lewis G. Birth weight and psychological distress at age 45-51 years: results from the Aberdeen Children of the 1950s cohort study. Br J Psychiatry 2005; 187: 21-8.

[58] Caspi A, McClay J, Moffitt TE, et al. Role of genotype in the cycle of violence in maltreated children.[see comment]. Science 2002; 297(5582): 851-4.

[59] Caspi A, Sugden K, Moffitt TE, et al. Influence of life stress on depression: moderation by a polymorphism in the 5-HTT gene.[see comment]. Science 2003; 301(5631): 386-9.

[60] Craske MG, Poulton R, Tsao JC, Plotkin D. Paths to panic disorder/agoraphobia: an exploratory analysis from age 3 to 21 in an unselected birth cohort. J Am Acad Child Adolesc Psychiatry 2001; 40(5): 556-63.

[61] Polanczyk G, Caspi A, Williams B, et al. Protective effect of CRHR1 gene variants on the development of adult depression following childhood maltreatment: replication and extension. Arch Gen Psychiatry 2009; 66(9): 978-85. 
[62] Paykel ES. Life events: effects and genesis. Psychol Med 2003; 33(7): 1145-8.

[63] Portin P, Alanen YO. A critical review of genetic studies of schizophrenia. I. Epidemiological and brain studies. [Review] [55 refs]. Acta Psychiatr Scand 1997; 95(1): 1-5.

[64] Zammit S, Rasmussen F, Farahmand B, et al. Height and body mass index in young adulthood and risk of schizophrenia: a longitudinal study of 1347520 Swedish men. Acta Psychiatr Scand 2007; 116(5): 378-85

[65] Rasmussen F. Paternal age, size at birth, and size in young adulthood - risk factors for schizophrenia. Eur J Endocrinol 2006; 155: S65-9.

[66] Isohanni M, Murray GK, Jokelainen J, Croudace T, Jones PB. The persistence of developmental markers in childhood and adolescence and risk for schizophrenic psychoses in adult life. A 34-year follow-up of the Northern Finland 1966 birth cohort. Schizophr Res 2004; 71(2-3): 213-25.

[67] Isohanni M, Lauronen E, Moilanen K, et al. Predictors of schizophrenia - Evidence from the northern Finland 1966 birth cohort and other sources. Br J Psychiatry 2005; 187: S4-7.

[68] Erlenmeyer-Kimling L, Rock D, Roberts SA, et al. Attention, memory, and motor skills as childhood predictors of schizophreniarelated psychoses: the New York High-Risk Project. Am J Psychiatry $2000 ; 157(9): 1416-22$.

[69] Kapur S. New Genes for Schizophrenia. BBC News on Line 2011.

[70] Merikangas KR, Risch N. Will the genomics revolution revolutionize psychiatry? Am J Psychiatry 2003; 160(4): 625-35.

[71] Kendler KS. "A gene for...": the nature of gene action in psychiatric disorders. Am J Psychiatry 2005; 162(7): 1243-52.

[72] Jones P. Childhood motor milestones and IQ prior to adult schizophrenia: Results from a 43 year old British birth cohort. Psychiatr Fenn 1995; 26: 63-80

[73] Jones PB, Tarrant CJ. Specificity of developmental precursors to schizophrenia and affective disorders. [Review] [32 refs]. Schizophr Res 1999; 39(2): 121-5.

[74] Jones PB. The early origins of schizophrenia. [Review] [91 refs]. Br Med Bull 1997; 53(1): 135-55.

[75] Geddes J, Lawrie S. Obstetric complications and schizophrenia: a meta-analysis. Br J Psychiatry 1995; 167: 786-93.

[76] Jones P, Rodgers B, Murray R, Marmot M. Child development risk factors for adult schizophrenia in the British 1946 birth cohort. Lancet 1994; 344(8934): 1398-402.

[77] Wahlbeck K, Forsen T, Osmond C, Barker DJP, Eriksson JG. Association of schizophrenia with low maternal body mass index, small size at birth, and thinness during childhood. Arch Gen Psychiatry $2001 ; 58(1)$ : 48-52.

[78] Gunnell D, Rasmussen F, Fouskakis D, Tynelius P, Harrison G. Patterns of fetal and childhood growth and the development of psychosis in young males: A cohort study. Am J Epidemiol 2003; 158(4): 291-300.

[79] Hoek H, Brown AS, Susser ES. The Dutch famine and schizophrenia spectrum disorders. Soc Psychiatry Psychiatr Epidemiol 1998; 33: 373-9.

[80] Susser E, Brown A, Matte T. Prenatal antecedents of neuropsychiatric disorder over the life course: Collaborative studies of United States birth cohorts. In: Rapoport JL, Ed. Childhood onset of "adult" psychopathology: Clinical and research advances.Washington DC: American Psychiatric Publishing Inc. 2000; pp. 121-46.

[81] Moilanen K, Jokelainen J, Jones PB, Hartikainen AL, Jarvelin MR, Isohanni $\mathrm{M}$. Deviant intrauterine growth and risk of schizophrenia: a 34-year follow-up of the Northern Finland 1966 Birth Cohort. Schizophr Res 2010; 124(1-3): 223-30.

[82] Hultman CM, Ohman A, Cnattingius S, Wieselgren IM, Lindstrom LH. Prenatal and neonatal risk factors for schizophrenia. Br J Psychiatry $1997 ; 170: 128-33$.

[83] Cannon M, Jones PB, Murray RM. Obstetric complications and schizophrenia: historical and meta-analytic review. Am J Psychiatry 2002; 159: 1080-92.

[84] Kagan J, Zentner M. Early childhood predictors of adult psychopathology. Harv Rev Psychiatry 1996; 3(6): 341-50.

[85] Ellison Z, Van Os J, Murray R. Special feature: childhood personality characteristics of schizophrenia: manifestations of, or risk factors for, the disorder?. [Review] [69 refs]. J Pers Dis 1998; 12(3): $247-61$.
[86] Welham J, Isohanni M. The antecedants of Schizophrenia: a review of birth cohort studies. Schizophr Bull 2009; 35(3): 603-23.

[87] Tarrant CJ, Jones PB. Precursors to schizophrenia: do biological markers have specificity? [see comment]. [Review] [103 refs]. Can J Psychiatry 1999; 44(4): 335-49.

[88] Schubert EW, Henriksson KM, McNeil TF. A prospective study of offspring of women with psychosis: visual dysfunction in early childhood predicts schizophrenia-spectrum disorders in adulthood. Acta Psychiatr Scand 2005; 112(5): 385-93.

[89] Perrin MA, Chen H, Sandberg DE, Malaspina D, Brown AS. Growth trajectory during early life and risk of adult schizophrenia. Br J Psychiatry 2007; 191: 512-20.

[90] Rodgers B. Behaviour and personality in childhood as predictors of adult psychiatric disorder. J Child Psychol Psychiatry Allied Discipline 1990; 31(3): 393-414.

[91] Walker E, Lewine EJ. Prediction of adult-onset schizophrenia from childhood movies of the patients. Am J Psychiatry 1990; 147: 1052-6.

[92] Poulton R, Caspi A, Moffitt TE, Cannon M, Murray RM, Harrington H. Children's self-reported psychotic symptoms and adult schizophreniform disorder. Arch Gen Psychiatry 2000; 57: 1053-8.

[93] Jaffee SR, Moffitt TE, Caspi A, Fombonne E, Poulton R, Martin J. Differences in early childhood risk factors for juvenile-onset and adult-onset depression.[see comment]. Arch Gen Psychiatry 2002; 59(3): 215-22.

[94] Cannon M, Moffitt TE, Caspi A, Murray RM, Harrington H, Poulton R. Neuropsychological performance at the age of 13 years and adult schizophreniform disorder: prospective birth cohort study. $\mathrm{Br}$ J Psychiatry 2006; 189: 463-4

[95] Koenen KC, Moffitt TE, Roberts AL, et al. Childhood IQ and adult mental disorders: a test of the cognitive reserve hypothesis. Am J Psychiatry 2009; 166: 50-7.

[96] Isohanni I, Jarvelin MR, Nieminen P, et al. School performance as a predictor of psychiatric hospitalization in adult life. A 28-year follow-up in the Northern Finland 1966 Birth Cohort. Psychol Med 1998; 28(4): 967-74.

[97] Lahti J, Raikkonen K, Sovio U, et al. Early-life origins of schizotypal traits in adulthood. Br J Psychiatry 2009; 195(2): 132-7.

[98] Brown AS, Cohen P, Harkavy-Friedman J, et al. Prenatal rubella, premorbid abnormalities, and adult schizophrenia. Biol Psychiatry 2001; 49(6): 473-86.

[99] Abrahao AL, Focaccia R, Gattaz WF. Childhood meningitis increases the risk for adult schizophrenia. World J Biol Psychiatry 2005; 6: 44-8.

[100] Rantakallio P, Jones P, Moring J, von Wendt L. Association between central nervous system infections during childhood and adult-onset schizophrenia and other psychoses. Int J Epidemiol 1997; 26: 837-43.

[101] Westergaard D, Mortensen P, Pedersen C, Wohlfarth J, Melbye M. Exposure to pre-natal and childhood infections and the risk of schizophrenia. Arch Gen Psychiatry 1999; 56: 993-8.

[102] Jokela M, Elovainio M, Singh-Manoux A, Kivimaki M. IQ, socioeconomic status, and early death: The US National Longitudinal Survey of Youth. Psychosom Med 2009; 71(3): 322-8.

[103] Jokela M, Batty GD, Deary IJ, Gale CR, Kivimaki M. Low childhood IQ and early adult mortality: the role of explanatory factors in the 1958 British Birth Cohort. Pediatrics 2009; 124(3): e380-8.

[104] Aylward E, Walker E, Battes B. Intelligence and schizophrenia: meta-analysis of the research. Schizophr Bull 1984; 10: 430-59.

[105] Cannon M, Jones P, Huttunen MO, Tanskanen A, Murray RM. Motor co-ordination deficits as predictors of schizophrenia among Finnish school children. Hum Psychopharmacol 1999; 14(7): 4917.

[106] Cannon M, Jones P, Huttunen MO, et al. School performance in Finnish children and later development of schizophrenia - A population-based longitudinal study. Arch Gen Psychiatry 1999; 56(5): 457-63.

[107] Szoke A, Trandafir A, Dupont ME, Meary A, Schurhoff F, Leboyer M. Longitudinal studies of cognition in schizophrenia: metaanalysis. Br J Psychiatry 2008; 192(4): 248-57.

[108] Murray GK, Jones PB. Infant motor development and adult cognitive functions in schizophrenia. Schizophr Res 2006; 81(1): 65-74.

[109] Costello EJ, Worthman C, Erkanli A, Angold A. Prediction from low birth weight to female adolescent depression: a test of competing hypotheses. Arch Gen Psychiatry 2007; 64(3): 338-44. 
[110] Gudmundsson P, Andersson S, Gustafson D, et al. Depression in Swedish women: relationship to factors at birth. Eur J Epidemiol 2011; 26(1): 55-60.

[111] Cunnane SC, Crawford MA. Survival of the fattest: fat babies were the key to evolution of the large human brain. Comp Biochem Physiol Part A Mol Integr Physiol 2003; 136(1): 17-26.

[112] Lahti J, Raikkonen K, Pesonen AK, et al. Prenatal growth, postnatal growth and trait anxiety in late adulthood - the Helsinki Birth Cohort Study. Acta Psychiatr Scand 2010; 121(3): 227-35.

[113] Zammit S, Allebeck P, David AS, et al. A longitudinal study of premorbid IQ Score and risk of developing schizophrenia, bipolar disorder, severe depression, and other nonaffective psychoses. Arch Gen Psychiatry 2004; 61(4): 354-60.

[114] Gale CR, Batty GD. Intelligence in early adulthood and subsequent hospitalization for mental disorders. Epidemiology 2010; 21(1): 707.

[115] Martin LT, Kubzansky LD, LeWinn KZ, Lipsitt LP, Satz P, Buka SL. Childhood cognitive performance and risk of generalized anxiety disorder. Int J Epidemiol 2007; 36(4): 769-75.

[116] Moran P, Klinteberg BAF. Childhood intelligence predicts hospitalization with personality disorder in adulthood: evidence from a population-based study in sweden. J Pers Disord 2009; 23(5): 53540

[117] Gravseth HM, Mehlum L. Suicide in young Norwegians in a life course perspective: population-based cohort study. J Epidemiol Communit Health 2010; 64(5): 407-12.

[118] McKinlay A, Grace R, Horwood J, Fergusson D, MacFarlane M. Adolescent psychiatric symptoms following preschool childhood mild traumatic brain injury: evidence from a birth cohort. J Head Trauma Rehabil 2009; 24(3): 221-7.

[119] Van Os J, Jones PB. Neuroticism as a risk factor for schizophrenia. Psychol Med 2001; 31(6): 1129-34.

[120] Fergusson DM, Beautrais AL, Horwood LJ. Vulnerability and resiliency to suicidal behaviours in young people. Psychol Med 2003; 33(1): 61-73.

[121] Fergusson DM, Horwood LJ. Does cannabis use encourage other forms of illicit drug use?[see comment]. Addiction 2000; 95(4): 505-20.

[122] Johnson JG, Cohen P, Kasen S, Smailes E, Brook JS. Association of maladaptive parental behavior with psychiatric disorder among parents and their offspring. Arch Gen Psychiatry 2001; 58(5): 45360

[123] Done DJ, Crow TJ, Johnstone EC, Sacker A. Childhood antecedents of schizophrenia and affective illness: social adjustment at ages 7 and 11.[see comment]. Br Med J 1994; 309(6956): 699-703.

[124] Palosaari U, Aro H, Laippala P. Parental divorce and depression in young adulthood: Adolescents' closeness to parents and self-esteem as mediating factor. Acta Psychiatr Scand 1996; 93(1): 20-6.

[125] Neeleman J, Sytema S, Wadsworth M. Propensity to psychiatric and somatic ill-health: evidence from a birth cohort. Psychol Med 2002; 32(5): 793-803.

[126] Rodgers B. Models of stress, vulnerability and affective disorder. J Affect Disord 1991; 21(1): 1-13.

[127] Rodgers B. Reported parental behaviour and adult affective symptoms. 2. Mediating factors. Psychol Med 1996; 26(1): 63-77.

[128] Kuh D, Hardy R, Rodgers B, Wadsworth ME. Lifetime risk factors for women's psychological distress in midlife. Soc Sci Med 2002; 55(11): 1957-73.

[129] Neeleman J, Wessely S, Wadsworth M. Predictors of suicide, accidental death, and premature natural death in a general-population birth cohort.[see comment]. Lancet 1998; 351(9096): 93-7.

[130] Sullaway FJ. Birth order and evolutionary psychology: a metaanalytic overview. Psychol Inq 1995; 6: 75-80.

[131] Kendler K, Aggen S, Jacobson K, Neale M. Does the level of family dysfunction moderate the impact of genetic factors on the presonailty trait of neuroticism? Psychol Med 2003; 33: 817-25.

[132] Van Os J, Jones PB. Early risk factors and adult person-environment relationships in affective disorder. Psychol Med 1999; 29(5): 1055-67.

[133] Gale CR, Batty GD, Deary IJ. Locus of control at age 10 years and health outcomes and behaviors at age 30 years: the 1970 British Cohort Study. Psychosom Med 2008; 70(4): 397-403.

[134] Acarturk C, Smit F, de GR, van SA, Ten HM, Cuijpers P. Incidence of social phobia and identification of its risk indicators: a model for prevention. Acta Psychiatr Scand 2009; 119(1): 62-70.
[135] Richards M, Huppert FA. Do positive children become positive adults? Evidence from a longitudinal birth cohort study. J Posit Psychol 2011; 6(1): 75-87.

[136] Fergusson DM, Goodwin RD, Horwood LJ. Neuroticism in adolescence and psychotic symptoms in adulthood. Psychol Med 2003; 33(6): 1089-97.

[137] Malmberg A, Lewis G, David A, Allebeck P. Pre-morbid adjustment and personality in people with schizophrenia. Br J Psychiatry 1998; 172: 308-13.

[138] Kosterman R, Hawkins JD, Mason WA, Herrenkohl TI, Lengua LJ, McCauley E. Assessment of behavior problems in childhood and adolescence as predictors of early adult depression. J Psychopathol Behav Assess 2009; 32(1): 118-27.

[139] Loeber R, Hay D. Key issues in the development of aggression and violence from childhood to early adulthood. Annu Rev Psychol 1997; 48: 371-410.

[140] Pajer KA. What happens to "bad" girls? A review of the adult outcomes of antisocial adolescent girls. Am J Psychiatry 1998; 155(7): 862-70.

[141] Patterson GR, DeBaryshe BD, Ramsey E. A developmental perspective on antisocial behavior. Am Psychol 1989; 44(2): 329-35.

[142] Rutter M. Relationships between mental disorders in childhood and adulthood. Acta Psychiatr Scand 1995; 91(2): 73-85.

[143] Fergusson DM, Horwood LJ, Ridder EM. Show me the child at seven: the consequences of conduct problems in childhood for psychosocial functioning in adulthood. J Child Psychol Psychiatry 2005; 46(8): 837-49.

[144] Fergusson DM, Horwood LJ. The structure, stability and correlations of the trait components of conduct disorder, attention deficit and anxiety/withdrawal reports. J Child Psychol Psychiatry 1993 ; 34(5): 749-66.

[145] Jokela M, Ferrie J, Kivimaki M. Childhood problem behaviors and death by midlife: the British National Child Development Study. J Am Acad Child Adolesc Psychiatry 2009; 48(1): 19-24.

[146] Rutter M. Isle of Wight revisited: twenty-five years of child psychiatric epidemiology. [Review] [325 refs]. J Am Acad Child Adolesc Psychiatry 1989; 28(5): 633-53.

[147] Biederman J, Petty C, Faraone SV, et al. Childhood antecedents to panic disorder in referred and nonreferred adults. J Child Adolesc Psychopharmacol 2005; 15(4): 549-61.

[148] Farrington DP. The Twelfth Jack Tizard Memorial Lecture. The development of offending and antisocial behaviour from childhood: key findings from the Cambridge Study in Delinquent Development. J Child Psychol Psychiatry Allied Disciplines 1995; 36(6): 929-64.

[149] Champion LA, Goodall G, Rutter M. Behaviour problems in childhood and stressors in early adult life. I. A 20 year follow-up of London school children. Psychol Med 1995; 25(2): 231-46.

[150] Schoon I, Sacker A, Bartley M. Socio-economic adversity and psychosocial adjustment: a developmental-contextual perspective. Soc Sci Med 2003; 57(6): 1001-15.

[151] Arseneault L, Moffitt TE, Caspi A, Taylor PJ, Silva PA. Mental disorders and violence in a total birth cohort: results from the Dunedin Study. Arch Gen Psychiatry 2000; 57(10): 979-86.

[152] Arseneault L, Cannon M, Murray R, Poulton R, Caspi A, Moffitt TE. Childhood origins of violent behaviour in adults with schizophreniform disorders. Br J Psychiatry 2003; 183: 520-5.

[153] Moffitt TE, Caspi A, Harrington H, Milne BJ. Males on the lifecourse-persistent and adolescence-limited antisocial pathways: follow-up at age 26 years. Dev Psychopathol 2002; 14(1): 179-207.

[154] Dalsgaard S, Mortensen PB, Frydenberg M, Thomsen PH. Conduct problems, gender and adult psychiatric outcome of children with attention-deficit hyperactivity disorder. Br J Psychiatry 2002; 181 : 416-21.

[155] Babinski LM, Hartsough CS, Lambert NM. Childhood conduct problems, hyperactivity-impulsivity, and inattention as predictors of adult criminal activity. J Child Psychol Psychiatry Allied Disciplines 1999; 40(3): 347-55.

[156] Mason WA, Kosterman R, Hawkins JD, Herrenkohl TI, Lengua LJ, McCauley E. Predicting depression, social phobia, and violence in early adulthood from childhood behavior problems. J Am Acad Child Adolesc Psychiatry 2004; 43(3): 307-15.

[157] Ensminger ME, Hanson SG, Riley AW, Juon HS. Maternal psychological distress: Adult sons' and daughters' mental health and educational attainment. J Am Acad Child Adolesc Psychiatry 2003; 42(9): 1108-15. 
[158] Appleyard K, Egeland B, van Dulmen MH, Sroufe LA. When more is not better: the role of cumulative risk in child behavior outcomes. J Child Psychol Psychiatry Allied Disciplines 2005; 46(3): 235-45.

[159] Heijmens Visser JH, van der Ende J, Koot HM, Verhulst FC. Predictors of psychopathology in young adults referred to mental health services in childhood or adolescence. Br J Psychiatry 2000; 177: 59-65.

[160] Arseneault L, Cannon M, Poulton R, Murray R, Caspi A, Moffitt TE. Cannabis use in adolescence and risk for adult psychosis: longitudinal prospective study. Br Med J 2002; 324(7374): 1212-3.

[161] Macleod J, Oakes R, Copello A, et al. Psychological and social sequelae of cannabis and other illicit drug use by young people: a systematic review of longitudinal, general population studies.[see comment]. [Review] [104 refs]. Lancet 2004; 363(9421): 1579-88.

[162] Hayatbakhsh MR, Najman JM, Bor W, O'Callaghan MJ, Williams GM. Multiple risk factor model predicting cannabis use and use disorders: a longitudinal study. Am J Drug Alcohol Abuse 2009; 35(6): 399-407.

[163] Nansel TR, Craig W, Overpeck MD, Saluja G, Ruan WJ. Crossnational consistency in the relationship between bullying behaviors and psychosocial adjustment. Arch Pediatr Adolesc Med 2004; 158(8): 730-6.

[164] Undheim AM, Sund AM. Prevalence of bullying and aggressive behavior and their relationship to mental health problems among 12- to 15-year-old Norwegian adolescents. Eur Child Adolesc Psychiatry 2010; 19(11): 803-11.

[165] Sourander A, Ronning J, Brunstein-Klomek A, et al. Childhood bullying behavior and later psychiatric hospital and psychopharmacologic treatment: findings from the Finnish 1981 birth cohort study. Arch Gen Psychiatry 2009; 66(9): 1005-12.

[166] Lien L, Green K, Welander-Vatn A, Bjertness E. Mental and somatic health complaints associated with school bullying between 10 th and 12th grade students; results from cross sectional studies in Oslo, Norway. Clin Pract Epidemiol Ment Health 2009; 5: 6.

[167] Olweus D, Limber SP, Mihalic S. The Bullying Prevention Program: Blueprints for Violence Prevention. vol. 10. Boulder,CO,USA: Center for the Study and Prevention of Violence 1999.

[168] Olweus D, Limber SP. The olweus bullying prevention program: implementation and evaluation over two decades. In: Jimerson SR, Swearer SM, Espelage DL, Eds. The International Handbook of School Bullying 2010. $1^{\text {st }}$ ed. New York: Routledge 2010.

[169] Smith PK, Pepler D, Rigby K. Bullying in schools: How successful can interventions be? Cambridge,UK: Cambridge University Press 2004.

[170] Solberg ME, Olweus D, Endresen IM. Bullies and victims at school: are they the same pupils? Br J Educ Psychol 2007; 77(Pt 2): 441-64.

[171] Duke NN, Pettingell SL, McMorris BJ, Borowsky IW. Adolescent violence perpetration: associations with multiple types of adverse childhood experiences. Pediatrics 2010; 125(4): e778-86.

[172] Klomek AB, Sourander A, Gould M. The association of suicide and bullying in childhood to young adulthood: a review of crosssectional and longitudinal research findings. Can J Psychiatry 2010; 55(5): 282-8.

[173] Klomek AB, Sourander A, Niemela S, et al. Childhood bullying behaviors as a risk for suicide attempts and completed suicides: a population-based birth cohort study. J Am Acad Child Adolesc Psychiatry 2009; 48(3): 254-61.

[174] Radford L, Corral S, Bradley C, et al. Child abuse and neglect in the UK today. London: NSPCC 2011.

[175] Public Safety Canada. Bullying Prevention: Nature and Extent of Bullying in Canada. Ottowa: Canadian National Crime Prevention Centre 2012.

[176] Fryers T, Brugha T, Melzer D, Jenkins R. The distribution of the common mental disorders; social inequalities in Europe. Clin Pract Epidemiol Ment Health 2005; 1: 14.

[177] Pless IB, Cripps HA, Wadsworth. Chronic physical illness in childhood: Psychological and social effects in adolescence and adult life. Dev Med Child Neurol 1989; 31(6): 746-55.

[178] Maughan B, Lindelow M. Secular change in psychosocial risks: the case of teenage motherhood. Psychol Med 1997; 27(5): 1129-44.

[179] Lehtinen H, Raikkonen K. School performance in childhood and adolescence as a predictor of depressive symptoms in adulthood. Sch Psychol Int 2006; 27 (3): 281-95.
[180] Conger R, Conger K, Elder G, Lorenz F, Simons R, Whitbeck L. A family process model of economic hardship and adjustment of early adolescent boys. Child Dev 1992; 63: 526-41.

[181] MacCabe JH, Lambe MP, Cnattingius S, et al. Scholastic achievement at age 16 and risk of schizophrenia and other psychoses: a national cohort study. Psychol Med 2008; 38(8): 1133-40.

[182] Almquist Y. Peer status in school and adult disease risk: a 30-year follow-up study of disease-specific morbidity in a Stockholm cohort. J Epidemiol Communit Health 2009; 63(12): 1028-34.

[183] Hardt J, Rutter M. Validity of adult retrospective reports of adverse childhood experiences: Review of the evidence. J Child Psychol Psychiatry Allied Disciplines 2004; 45(2): 260-73.

[184] Hardt J, Vellaisamy P, Schoon I. Sequelae of prospective versus retrospective reports of adverse childhood experiences. Psychol Rep 2010; 107(2): 425-40.

[185] Wadsworth MEJ, Kuh DJL. Childhood influences on adult health: A review of resent work from the British 1946 national birth cohort study, the MRC National Survey of Health and Development. Paediatr Perinat Epidemiol 1997; 11(1): 2-20.

[186] Richards M, Wadsworth ME. Long term effects of early adversity on cognitive function. Arch Dis Child 2004; 89(10): 922-7.

[187] Power C, Stansfeld SA, Matthews S, Manor O, Hope S. Childhood and adulthood risk factors for socio-economic differentials in psychological distress: evidence from the 1958 British birth cohort. Soc Sci Med 2002; 55(11): 1989-2004.

[188] Rodgers B. Adult affective disorder and early environment. Br J Psychiatry 1990; 157: 539-50.

[189] Anda RF, Felitti VJ. The enduring effects of abuse and related adverse experiences in childhood. A convergence of evidence from neurobiology and epidemiology. Eur Arch Psychiatry Clin Neurosci 2006; 256(3): 174-86.

[190] Anda RF, Brown DW, Felitti VJ, Bremner JD, Dube SR, Giles WH. Adverse childhood experiences and prescribed psychotropic medications in adults. Am J Prev Med 2007; 32(5): 389-94.

[191] Rosenman S, Rodgers B. Childhood adversity and adult personality. Aust NZ J Psychiatry 2006; 40(5): 482-90.

[192] Stansfeld S, Head J, Bartley M, Fonagy P. Social position, early deprivation and the development of attachment. Soc Psychiatry Psychiatr Epidemiol 2008; 43(7): 516-26.

[193] McLaughlin KA, Green JG, Gruber MJ, Sampson NA, Zaslavsky AM, Kessler RC. Childhood adversities and adult psychiatric disorders in the national comorbidity survey replication II: associations with persistence of DSM-IV disorders. Arch Gen Psychiatry 2010; 67(2): 124-32.

[194] Green JG, McLaughlin KA, Berglund PA, et al. Childhood adversities and adult psychiatric disorders in the national comorbidity survey replication I: associations with first onset of DSM-IV disorders. Arch Gen Psychiatry 2010; 67(2): 113-23.

[195] McLaughlin KA, Green JG, Gruber MJ. Childhood adversities and adult psychopathology in the National Co-morbidity Survey Replication (NCS-R) III: associations with functional impairment related to DSM-IV disorders. Psychol Med 2012; 40(5): 847-59.

[196] Benjet C, Borges G, Medina-Mora ME. Chronic childhood adversity and onset of psychopathology during three life stages: childhood, adolescence and adulthood. J Psychiatr Res 2010; 44(11): 732-40.

[197] Pirkola S, Isometsa E, Aro H, et al. Childhood adversities as risk factors for adult mental disorders: results from the Health 2000 study. Soc Psychiatry Psychiatr Epidemiol 2005; 40(10): 769-77.

[198] Juon H-S, Ensminger ME. Childhood, adolescent, and young adult predictors of suicidal behaviors: A prospective study of African Americans. J Child Psychol Psychiatry 1997; 38(5): 553-63.

[199] Enns MW, Cox BJ, Afifi TO, de GR, Ten HM, Sareen J. Childhood adversities and risk for suicidal ideation and attempts: a longitudinal population-based study. Psychol Med 2006; 36(12): 1769-78.

[200] Lee S, Guo WJ. The prevalence of family childhood adversities and their association with first onset of DSM-IV disorders in metropolitan China. Psychol Med 2011; 41(1): 85-96.

[201] Kristensen P, Gravseth HM, Bjerkedal T. Influence of early life factors on social inequalities in psychiatric outcomes among young adult Norwegian men. Eur J Public Health 2010; 20(5): 517-23.

[202] Melchior M, Moffitt TE, Milne BJ, Poulton R, Caspi A. Why do children from socioeconomically disadvantaged families suffer from poor health when they reach adulthood? A life-course study. Am J Epidemiol 2007; 166(8): 966-74. 
[203] Power C, Manor O. Explaining social class differences in psychological health among young adults: a longitudinal perspective. Soc Psychiatry Psychiatr Epidemiol 1992; 27(6): 284-91.

[204] Schoon I, Montgomery SM. The relationship between early life experiences and adult depression. Z Psychosom Med Psychother 1997; 43(4): 319-33.

[205] Russell ST. Childhood developmental risk for teen childbearing in Britain. J Res Adolesc 2002; 12(3): 305

[206] Andersen TF. Persistence of social and health problems in the welfare state: a Danish cohort experience from 1948 to 1979. Soc Sci Med 1984; 18(7): 555-60.

[207] Costello E, Compton S, Keeler G, Angold A. Relationships between poverty and psychopathology; a natural experiment. J Am Med Assoc 2003; 290: 2023-9.

[208] Wicks S, Hjern A, Gunnell D, Lewis G, Dalman C. Social adversity in childhood and the risk of developing psychosis: a national cohort study. Am J Psychiatry 2005; 162(9): 1652-7.

[209] Anselmi L, Barros FC. Prevalence and early determinants of common mental disorders in the 1982 birth cohort, Pelotas, Southern Brazil. Rev Saude Publica 2008; 2: 26-33.

[210] Vinnerljung B, Hjern A, Lindblad F. Suicide attempts and severe psychiatric morbidity among former child welfare clients--a national cohort study. J Child Psychol Psychiatry 2006; 47(7): 72333

[211] Stansfeld SA, Clark C, Rodgers B, Caldwell T, Power C. Childhood and adulthood socio-economic position and midlife depressive and anxiety disorders. Br J Psychiatry 2008; 192(2): 152-3.

[212] Power C, Atherton K, Strachan DP, et al. Life-course influences on health in British adults: effects of socio-economic position in childhood and adulthood. Int J Epidemiol 2007; 36(3): 532-9.

[213] Lalor K, McElvaney R. Child sexual abuse, links to later sexual exploitation/high-risk sexual behavior, and prevention/treatment programs. Trauma Violence Abuse 2010; 11(4): 159-77.

[214] Maniglio R. The impact of child sexual abuse on health: a systematic review of reviews. Clin Psychol Rev 2009; 29(7): 647-57.

[215] Neumann DA, Houskamp BM, Pollack VE, Briere J. The longterm sequellae of childhood sexual abuse in women: A metaanalytic review. Child Maltreat 1996; 1: 1-16.

[216] Sharpe D, Faye C. Non-epileptic seizures and child sexual abuse: a critical review of the literature. Clin Psychol Rev 2006; 26(8): $1020-40$

[217] Korkeila J, Vahtera J, Nabi H, et al. Childhood adversities, adulthood life events and depression. J Affect Disord 2010; 127(1-3): 130-8.

[218] Paykel ES. The evolution of life events research in psychiatry. [Review] [82 refs]. J Affect Disord 2001; 62(3): 141-9.

[219] Rutter M. Cause and course of psychopathology: some lessons from longitudinal data. Paediatr Perinat Epidemiol 1993; 7(2): 10520 .

[220] Paykel ES. Stress and affective disorders in humans. [Review] [81 refs]. Semin Clin Neuropsychiatry 2001; 6(1): 4-11.

[221] Phillips NK, Hammen CL, Brennan PA, Najman JM, Bor W. Early adversity and the prospective prediction of depressive and anxiety disorders in adolescents. J Abnorm Child Psychol 2005; 33(1): 1324.

[222] Heinonen K, Keltikangas-Jarvinen L, Pesonen A-K, Raikkonen K, Schubert C, Viikari J. Parental reports of global physical health at ages 3 and 6 predict self-reported depressive symptoms 17 years later. Br J Dev Psychol 2004; 22(3): 459-69.

[223] Power C, Peckham C. Childhood morbidity and adulthood ill health. J Epidemiol Communit Health 1990; 44(1): 69-74.

[224] Pless IB, Power C, Peckham CS. Long-term psychosocial sequelae of chronic physical disorders in childhood. Pediatrics 1993; 91(6): 1131-6.

[225] Fearon P, Hotopf M. Relation between headache in childhood and physical and psychiatric symptoms in adulthood: national birth cohort study. Br Med J 2005; 322(7295): 1145-8.

[226] Viner R, Hotopf M. Childhood predictors of self reported chronic fatigue syndrome/myalgic encephalomyelitis in adults: national birth cohort study.[see comment]. Br Med J 2004; 329(7472): 941.

[227] Veijola J, Maki P, Joukamaa M, Laara E, Hakko H, Isohanni M. Parental separation at birth and depression in adulthood: a longterm follow-up of the Finnish Christmas Seal Home Children. Psychol Med 2004; 34(2): 357-62.

[228] Hawker DSJ, Boulton MJ. Twenty years' research on peer victimization and psychosocial maladjustment. A meta-analytic review of cross-sectional studies. J Child Psychol Psychiatry 2000; 41: 44155.

[229] Solberg ME, Olweus D. Prevalence estimation of school bullying with the Olweus Bully/Victim Questionnaire. Aggress Behav 2003; 29: 239-68.

[230] Olweus D. Bullying at school: What we know and what we can do. Cambridge,UK: Blackwell 1993.

[231] Gladstone GL, Parker GB, Malhi GS. Do bullied children become anxious and depressed adults?: A cross-sectional investigation of the correlates of bullying and anxious depression. J Nerv Ment Dis 2006; 194(3): 201-8.

[232] Fosse GK. Mental health of psychiatric outpatients bullied in childhood. PhD Thesis. Trondheim: Department of Neuroscience, Faculty of Medicine, Norwegian University of Science and Technology 2006.

[233] Lund R, Nielsen KK, Hansen DH, et al. Exposure to bullying at school and depression in adulthood: a study of Danish men born in 1953. Eur J Public Health 2009; 19(1): 111-6.

[234] Due P, Damsgaard MT, Lund R, Holstein BE. Is bullying equally harmful for rich and poor children?: a study of bullying and depression from age 15 to 27. Eur J Public Health 2009; 19(5): 464-9.

[235] Fergusson DM, Horwood LJ, Lynskey MT. Childhood sexual abuse and psychiatric disorder in young adulthood: II. Psychiatric outcomes of childhood sexual abuse. J Am Acad Child Adolesc Psychiatr 1996; 35(10): 1365-74.

[236] Weiss EL, Longhurst JG, Mazure CM. Childhood sexual abuse as a risk factor for depression in women: psychosocial and neurobiological correlates. Am J Psychiatry 1999; 156(6): 816-28.

[237] Weich S, Patterson J, Shaw R, Stewart-Brown S. Family relationships in childhood and common psychiatric disorders in later life: systematic review of prospective studies. Br J Psychiatry 2009; 194(5): 392-8.

[238] Nurcombe B. Child sexual abuse I: psychopathology. Aust NZ J Psychiatry 2000; 34(1): 85-91.

[239] Springer KW, Sheridan J, Kuo D, Carnes M. The long-term health outcomes of childhood abuse. An overview and a call to action.[see comment]. J Gen Intern Med 2003; 18(10): 864-70.

[240] Beckett H. 'Not a world away' The sexual exploitation of children and young people in Northern Ireland. Barnado's Northern Ireland: Belfast 2011

[241] Cutajar MC, Mullen PE, Ogloff JR, Thomas SD, Wells DL, Spataro J. Psychopathology in a large cohort of sexually abused children followed up to 43 years. Child Abuse Negl 2010; 34(11): 813-22.

[242] May-Chahal C, Cawson P. Measuring child maltreatment in the United Kingdom: a study of the prevalence of child abuse and neglect. Child Abuse Negl 2005; 29(9): 969-84.

[243] United Nations. Report of the independent expert for the United Nations study on violence against children. United Nations; 2006.

[244] Tajima EA, Herrenkohl TI, Huang B, Whitney SD. Measuring child maltreatment: A comparison of prospective parent reports and retrospective adolescent reports. Am J Orthopsychiatry 2004; 74(4): 424-35

[245] Rodgers B. Reported parental behaviour and adult affective symptoms. 1. Associations and moderating factors. Psychol Med 1996; 26(1): 51-61

[246] Mina E, Gallop R. Childhood sexual and physical abuse and adult self-harm and suicidal behaviour; a literature review. Can J Psychiatry 1998; 43: 793-800.

[247] Cicchetti D, Manly J. Operationalising child mal-treatment: developmental processes and outcomes. Editorial. Dev Psychopathol 2001; 13(4): 755-8.

[248] Widom CS, White HR. Problem behaviours in abused and neglected children grown up: Prevalence and co-occurrence of substance abuse, crime and violence. Crim Behav Ment Health 1997; 7(4): 287-310.

[249] Buist A. Childhood abuse, postpartum depression and parenting difficulties: a literature review of associations. Aus NZ J Psychiatry 1998; 32(3): 370-8.

[250] Spataro J, Mullen PE, Burgess PM, Wells DL, Moss SA. Impact of child sexual abuse on mental health: prospective study in males and females.[see comment]. Br J Psychiatry 2004; 184: 416-21.

[251] Fergusson DM, Lynskey MT, Horwood LJ. Childhood sexual abuse and psychiatric disorder in young adulthood: I. Prevalence of sexual abuse and factors associated with sexual abuse. J Am Acad Child Adolesc Psychiatry 1996; 35(10): 1355-64. 
[252] Fergusson DM, Boden JM, Horwood LJ. Exposure to childhood sexual and physical abuse and adjustment in early adulthood. Child Abuse Negl 2008; 32(6): 607-19.

[253] Thornberry T, Ireland TO, Smith CA. The importance of timing: the varying impact of childhood and adolescent mal-treatment on multiple problem outcomes. Dev Psychopathol 2001; 13(4): 95780.

[254] Malinosky-Rummell R, Hansen DJ. Long-term consequences of childhood physical abuse. Psychol Bull 1993; 114(1): 68-79.

[255] Roberts R, O'Connor T, Dunn J, Golding J. The effects of child sexual abuse in later family life; mental health, parenting and adjustment of offspring. Child Abuse Negl 2004; 28(5): 525.

[256] Zimrin H. A profile of survival. Child Abuse Negl 1986; 10(3): 339-49.

[257] Lynskey MT, Fergusson DM. Factors protecting against the development of adjustment difficulties in young adults exposed to childhood sexual abuse. Child Abuse Neglect 1997; 21(12): 1177-90.

[258] Collishaw S, Dunn J, O'Connor TG, Golding J. Maternal childhood abuse and offspring adjustment over time. Dev Psychopathol 2007;19(2):367-83.

[259] Sachs-Ericsson N, Cromer K, Hernandez A, Kendall-Tackett K. A review of childhood abuse, health, and pain-related problems: the role of psychiatric disorders and current life stress. J Trauma Dissociation $2009 ; 10(2)$ : 170-88.

[260] Noll JG, Trickett PK, Harris WW, Putnam FW. The cumulative burden borne by offspring whose mothers were sexually abused as children: descriptive results from a multigenerational study. J Interpers Violence 2009; 24: 424-49.

[261] CDC. Adverse Childhood Experiences Reported by Adults - Five States, 2009. Report No.: Morbidity and Mortality Weekly Report, December $17^{\text {th }}, 2009$. USA: Centres for Disease Control (CDC); 2010.

[262] Stouthamer-Loeber M, Loeber R, Homish DL, Wei E. Maltreatment of boys and the development of disruptive and delinquent behaviour. Dev Psychopathol 2001; 13(4): 941-56.

[263] Silverman AB, Reinherz HZ, Giaconia RM. The long-term sequelae of child and adolescent abuse: a longitudinal community study. Child Abuse Negl 1996; 20(8): 709-23.

[264] Cawson P, Wattam L, Brooker S, Kelly G. A study of the prevalence of child abuse and neglect. NSPCC, UK 2000.

[265] Bifulco A, Bernazzani O, Moran PM, Jacobs C. The childhood experience of care and abuse questionnaire (CECA.Q): validation in a community series. Br J Clin Psychol 2005; 44(Pt 4): 563-81.

[266] Geraerts E, Jelicic M, Merckelbach H. Symptom over-reporting and recovered memories of childhood sexual abuse. Law Hum Behav 2006; 30(5): 621-30.

[267] Sidebotham P, Heron J. Child maltreatment in the "children of the nineties": a cohort study of risk factors. Child Abuse Negl 2006; 30(5): 497-522.

[268] Rind B, Tromovitch P, Bauserman R. A meta-analytic examination of assumed properties of child sexual abuse using college samples. Psychol Bull 1998; 124(1): 22-53.

[269] Cutajar MC, Mullen PE, Ogloff JR, Thomas SD, Wells DL, Spataro J. Schizophrenia and other psychotic disorders in a cohort of sexually abused children. Arch Gen Psychiatry 2010; 67(11): 1114-9.

[270] DuMont KA, Widom CS, Czaja SJ. Predictors of resilience in abused and neglected children grown-up: the role of individual and neighborhood characteristics. Child Abuse Negl 2007; 31(3): 25574.

[271] Fisher HL, Jones PB, Fearon P, et al. The varying impact of type, timing and frequency of exposure to childhood adversity on its association with adult psychotic disorder. Psychol Med 2010; 40(12): 1967-78.

[272] Shevlin M, Dorahy MJ, Adamson G. Trauma and psychosis: an analysis of the National Comorbidity Survey. Am J Psychiatry 2007; 164(1): 166-9.

[273] Schurhoff F, Laguerre A. Self-reported childhood trauma correlates with schizotypal measures in schizophrenia but not bipolar pedigrees. Psychol Med 2009; 39(3): 365-70.

[274] Shevlin M, Houston JE, Dorahy MJ, Adamson G. Cumulative traumas and psychosis: an analysis of the national comorbidity survey and the British Psychiatric Morbidity Survey. Schizophr Bull 2008; 34(1): 193-9.
[275] Paolucci EO, Genuis ML, Violato C. A meta-analysis of the published research on the effects of child sexual abuse. J Psychol 2001; 135(1): 17-36.

[276] Stewart-Brown S, Shaw R. The roots of social capital; relationships in the home during childhood, and health in adult life. In: Morgan A, Swann C, Eds. Social capital and health: issues of definition, measurement and links to health. London: Health Development Agency 2004.

[277] Goodwin RD, Fergusson DM, Horwood LJ. Childhood abuse and familial violence and the risk of panic attacks and panic disorder in young adulthood. Psychol Med 2005; 35(6): 881-90.

[278] Fergusson DM, Horwood LJ. Exposure to interparental violence in childhood and psychosocial adjustment in young adulthood. Child Abuse Negl 1998; 22(5): 339-57.

[279] Brown J, Cohen P, Johnson JG, Smailes EM. Childhood abuse and neglect: specificity of effects on adolescent and young adult depression and suicidality.[see comment]. J Am Acad Child Adolesc Psychiatry 1999; 38(12): 1490-6.

[280] Dube S, Felitti M, Dong M, Giles W, Anda R. The impact of adverse childhood experiences on health problems: evidence from four birth cohorts dating back to 1900. Prev Med 2003; 37: 268-77.

[281] Horwitz AV, Widom CS, McLaughlin J, White HR. The impact of childhood abuse and neglect on adult mental health: a prospective study. J Health Soc Behav 2001; 42(2): 184-201.

[282] Clark C, Caldwell T, Power C, Stansfeld SA. Does the influence of childhood adversity on psychopathology persist across the lifecourse? A 45-year prospective epidemiologic study. Ann Epidemiol 2010; 20(5): 385-94.

[283] Adams RE, Bukowsky WM. Relationships with mothers and peers moderate the association between childhood sexual abuse and anxiety disorders. Child Abuse Negl 2007; 31: 645-56.

[284] Afifi TO, Boman J, Fleisher W, Sareen J. The relationship between child abuse, parental divorce, and lifetime mental disorders and suicidality in a nationally representative adult sample. Child Abuse Neg1 2009; 33: 139-47.

[285] Cougle JR, Timpano KR, Sachs-Ericsson N, Keough ME, Riccardi CJ. Examining the unique relationships between anxiety disorders and childhood physical and sexual abuse in the National Comorbidity Survey-Replication. Psychiatry Res 2010; 177(1-2): 150-5.

[286] Wiersma JE, Hovens JG, van OP, et al. The importance of childhood trauma and childhood life events for chronicity of depression in adults. J Clin Psychiatry 2009; 70(7): 983-9.

[287] Gault-Sherman M, Silver E, Sigfusdottir ID. Gender and the associated impairments of childhood sexual abuse: a national study of Icelandic youth. Soc Sci Med 2009; 69(10): 1515-22.

[288] Geschwind N, Peeters F, Jacobs N, et al. Meeting risk with resilience: high daily life reward experience preserves mental health Acta Psychiatr Scand 2010; 122(2): 129-38.

[289] Hovens JG, Wiersma JE, Giltay EJ, et al. Childhood life events and childhood trauma in adult patients with depressive, anxiety and comorbid disorders vs. controls. Acta Psychiatr Scand 2010; 122(1): 66-74.

[290] McCutcheon VV, Heath AC. Accumulation of trauma over time and risk for depression in a twin sample. Psychol Med 2009; 39(3): 431-41.

[291] McCutcheon VV, Sartor CE. Age at trauma exposure and PTSD risk in young adult women. J Trauma Stress 2011; 23(6): 811-4.

[292] McDonagh A, Friedman M, McHugo G, et al. Randomized trial of cognitive-behavioral therapy for chronic posttraumatic stress disorder in adult female survivors of childhood sexual abuse. J Consult Clin Psychol 2005; 73(3): 515-24.

[293] Brezo J, Paris J, Vitaro F, Hebert M, Tremblay RE, Turecki G. Predicting suicide attempts in young adults with histories of childhood abuse. Br J Psychiatry 2008; 193(2): 134-9.

[294] Joiner TE, Sachs-Ericsson NJ. Childhood physical and sexual abuse and lifetime number of suicide attempts: a persistent and theoretically important relationship. Behav Res Ther 2007; 45(3): 539-47.

[295] Cutajar MC, Mullen PE, Ogloff JR, Thomas SD, Wells DL, Spataro J. Suicide and fatal drug overdose in child sexual abuse victims: a historical cohort study. Med J Aust 2010; 192(4): 184-7.

[296] Fliege H, Lee JR, Grimm A, Klapp BF. Risk factors and correlates of deliberate self-harm behavior: a systematic review. J Psychosom Res 2009; 66(6): 477-93

[297] Klonsky ED, Moyer A. Childhood sexual abuse and non-suicidal self-injury: meta-analysis. Br J Psychiatry 2008; 192(3): 166-70. 
[298] Perepletchikova F, Kaufman J. Emotional and behavioral sequelae of childhood maltreatment. Curr Opin Pediatr 2010; 22(5): 610-5.

[299] Fossati A, Madeddu F, Maffei C. Borderline Personality Disorder and childhood sexual abuse: a meta-analytic study. J Pers Disord 1999; 13(3): 268-80.

[300] Caspi A, Vishne T, Sasson Y, Gross R, Livne A, Zohar J. Relationship between childhood sexual abuse and obsessive-compulsive disorder: case control study. Isr J Psychiatry Relat Sci 2008; 45(3): 177-82.

[301] Sanci L, Coffey C, Olsson C, Reid S, Carlin JB, Patton G. Childhood sexual abuse and eating disorders in females: findings from the Victorian Adolescent Health Cohort Study. Arch Pediatr Adolesc Med 2008; 162(3): 261-7.

[302] Bohn K, Doll HA, Cooper Z, O'Connor M, Palmer RL, Fairburn CG. The measurement of impairment due to eating disorder psychopathology. Behav Res Ther 2008; 46(10): 1105-10.

[303] Palmer RL, Oppenheimer R, Dignon A, Chaloner DA, Howells K. Childhood sexual experiences with adults reported by women with eating disorders: an extended series. Br J Psychiatry 1990; 156: 699-703.

[304] Reyes-Rodriguez ML, Von HA, Ulman TF, et al. Posttraumatic stress disorder in anorexia nervosa. Psychosom Med 2011; 73(6): 491-7.

[305] Jumper SA. A meta-analysis of the relationship of child sexual abuse to adult psychological adjustment. Child Abuse Negl 1995; 19(6): 715-28

[306] Thornberry TP, Henry KL, Ireland TO, Smith CA. The causal impact of childhood-limited maltreatment and adolescent maltreatment on early adult adjustment. J Adolesc Health 2010; 46(4): 359-65.

[307] Watson B, Halford WK. Classes of childhood sexual abuse and women's adult couple relationships. Violence Vict 2010; 25(4): 518-35.

[308] Ouyang L, Fang X, Mercy J, Perou R, Grosse SD. Attentiondeficit/hyperactivity disorder symptoms and child maltreatment: a population-based study. J Pediatr 2008; 153(6): 851-6.

[309] Briere J, Jordan CE. Childhood maltreatment, intervening variables, and adult psychological difficulties in women: an overview. Trauma Violence Abuse 2009; 10(4): 375-88.

[310] Masters A. Stuart, A life backwards. $2^{\text {nd }}$ ed. London: Harper Perennial 2006.

[311] Lima AR, Mello MF, Mari JJ. The role of early parental bonding in the development of psychiatric symptoms in adulthood. Curr Opin Psychiatry 2010; 23(4): 383-7.

[312] Huppert FA, Abbott RA. Parental practices predict psychological well-being in midlife: life-course associations among women in the 1946 British birth cohort. Psychol Med 2010; 40(9): 1507-18.

[313] Morgan Z, Brugha T, Fryers T, Stewart-Brown S. The effects of parent-child relationships on later life mental health status in two national birth cohorts. Soc Psychiatry Psychiatr Epidemiol 2012.

[314] Stewart-Brown SL, Fletcher L, Wadsworth ME. Parent-child relationships and health problems in adulthood in three UK national birth cohort studies. Eur J Public Health 2005; 15(6): 640-6.

[315] Patton GC, Coffey C, Posterino M, Carlin JB, Wolfe R. Parental 'affectionless control' in adolescent depressive disorder. Soc Psychiatry Psychiatr Epidemiol 2001; 36(10): 475-80.

[316] Johnson JG, Cohen P, Chen H, Kasen S, Brook JS. Parenting behaviors associated with risk for offspring personality disorder during adulthood. Arch Gen Psychiatry 2006; 63(5): 579-87.

[317] Gao Y, Raine A, Chan F, Venables PH, Mednick SA. Early maternal and paternal bonding, childhood physical abuse and adult psychopathic personality. Psychol Med 2010; 40(6): 1007-16.

[318] Sidebotham P, Golding J, Parents ASTALSo, Children. Child maltreatment in the "children of the nineties" a longitudinal study of parental risk factors. Child Abuse Negl 2001; 25(9): 1177-200.

[319] Fergusson DM, Woodward LJ. Maternal age and educational and psychosocial outcomes in early adulthood. J Child Psychol Psychiatry Allied Disciplines 1999; 40(3): 479-89.

[320] Nagin D, Pogarsky G, Farrington DP. Adolescent mothers and the criminal behaviour of their children. Law Soc Rev 1997; 31: 13762.

[321] Scaramella L, Conger R, Simons R, Whitbeck L. Predicting risk for pregnancy by late adolescence: a social contextual perspective. Dev Psychol 1998; 34: 1233-45.
[322] McLeod BD, Weisz JR, Wood JJ. Examining the association between parenting and childhood depression: A meta-analysis. Clin Psychol Rev 2007; 27: 986-1003.

[323] Yu ML, Ziviani J, Baxter J, Haynes M. Time use, parenting practice and conduct problems in four- to five-year-old Australian children. Aust Occup Ther J 2010; 57(5): 284-92.

[324] Peltonen K, Ellonen N, Larsen HB, Helweg-Larsen K. Parental violence and adolescent mental health. Eur Child Adolesc Psychiatry $2010 ; 19(11): 813-22$.

[325] Lynch SK, Turkheimer E, D'Onofrio BM, et al. A genetically informed study of the association between harsh punishment and offspring behavioral problems. J Fam Psychol 2006; 20(2): 190-8.

[326] Oddy WH, Kendall GE. The long-term effects of breastfeeding on child and adolescent mental health: a pregnancy cohort study followed for 14 years. J Pediatrics 2010; 156(4): 568-74.

[327] Barnes J, Stein A. Effects of parental psychiatric and physical illness on child development. In: Gelder M, Lopez-Ibor J, Andreasson N, Eds. New Oxford Textbook of Psychiatry.Oxford, UK: Oxford University Press 2000; pp. 1848-55.

[328] Beardslee WR, Versage EM, Gladstone TR. Children of affectively ill parents: a review of the past 10 years. J Am Acad Child Adolesc Psychiatry 1998; 37(11): 1134-41.

[329] Goodman SH, Gotlib IH. Children of depressed parents;alternative pathways to risk for psycho-pathology. Washington DC, USA: Am Psychol Assoc Press 2002.

[330] Niemi LT, Suvisaari JM, Haukka JK, Wrede G, Lonnqvist JK. Cumulative incidence of mental disorders among offspring of mothers with psychotic disorder. Results from the Helsinki HighRisk Study. Br J Psychiatry 2004; 185: 11-7.

[331] Lieb R, Isensee B, Hofler M, Wittchen HU. Parental depression and depression in offspring: evidence for familial characteristics and subtypes? J Psychiatr Res 2002; 36(4): 237-46.

[332] Lieb R, Merikangas KR, Hofler M, Pfister H, Isensee B, Wittchen HU. Parental alcohol use disorders and alcohol use and disorders in offspring: a community study. Psychol Med 2002; 32(1): 63-78.

[333] Lieb R, Isensee B, Hofler M, Pfister H, Wittchen HU. Parental major depression and the risk of depression and other mental disorders in offspring: a prospective-longitudinal community study. Arch Gen Psychiatry 2002; 59(4): 365-74.

[334] Weissman MM, Wickramaratne P, Nomura Y, Warner V, Pilowsky D, Verdeli H. Offspring of depressed parents: 20 years later. Am J Psychiatry 2006; 163(6): 1001-8.

[335] Rudisch B, Nemeroff CB. Epidemiology of comorbid coronary artery disease and depression. Biol Psychiatry 2003; 54(3): 227-40.

[336] Weissman MM, Wickramaratne P, Nomura Y, et al. Families at high and low risk for depression: a 3-generation study. Arch Gen Psychiatry 2005; 62(1): 29-36.

[337] Johnson JG, Cohen P, Gould MS, Kasen S, Brown J, Brook JS. Childhood adversities, interpersonal difficulties, and risk for suicide attempts during late adolescence and early adulthood. Arch Gen Psychiatry 2002; 59(8): 741-9.

[338] Johnson JG, Smailes E, Cohen P, Kasen S, Brook JS. Anti-social parental behaviour, problematic parenting and aggressive offspring behaviour during adulthood - A 25 -year longitudinal investigation. Br J Criminol 2004; 44(6): 915.

[339] Larzelere R, Patterson G. Parental management: mediator of the effect of socio-economic status on early delinquency. Criminology 1990; 28: 301-23.

[340] Kolvin I, Miller FJ, Fleeting M, Kolvin PA. Social and parenting factors affecting criminal-offence rates: Findings from the Newcastle Thousand Family Study (1947-1980). Br J Psychiatry 1988; 152: 80-90.

[341] Sadowski H, Ugarte B, Kolvin I, Kaplan C, Barnes J. Early life family disadvantages and major depression in adulthood. Br J Psychiatry $1999 ; 174: 112-20$.

[342] Loeber R, Hipwell A, Battista D, Sembower M, Stouthamer-Loeber M. Intergenerational transmission of multiple problem behaviors: prospective relationships between mothers and daughters. J Abnorm Child Psychol 2009; 37(8): 1035-48.

[343] Elgar FJ, Mills RS, McGrath PJ, Waschbusch DA, Brownridge DA. Maternal and paternal depressive symptoms and child maladjustment: the mediating role of parental behavior. J Abnorm Child Psychol 2007; 35(6): 943-55.

[344] Hayatbakhsh MR, Mamun AA, Najman JM, O'Callaghan MJ, Bor W, Alati R. Early childhood predictors of early substance use and 
substance use disorders: prospective study. Aust N Z J Psychiatry 2008; 42(8): 720-31.

[345] Knappe S, Lieb R. The role of parental psychopathology and family environment for social phobia in the first three decades of life. Depress Anxiety 2009; 26(4): 363-70.

[346] Knappe S, Beesdo K, Fehm L, Hofler M, Lieb R, Wittchen HU. Do parental psychopathology and unfavorable family environment predict the persistence of social phobia? J Anxiety Disord 2009; 23(7): 986-94

[347] Harold GT, Rice F, Hay DF, Boivin J, van de Bree M, Thapar A. Familial transmission of depression and antisocial behavior symptoms: disentangling the contribution of inherited and environmental factors and testing the mediating role of parenting. Psychol Med 2011; 41(6): 1175-85.

[348] Maughan B, McCarthy G. Childhood adversities and psychosocial disorders. Br Med Bull 1997; 53(1): 156-69.

[349] Amato P, Keith B. Parental divorce and adult well-being; a metaanalysis. J Marriage Fam 1991; 53: 43-58.

[350] Pfeffer CR, Altemus M. Salivary cortisol and psychopathology in children bereaved by the september 11, 2001 terror attacks. Biol Psychiatry 2007; 61(8): 957-65.

[351] Jacobs JR, Bovasso GB. Re-examining the long-term effects of experiencing parental death in childhood on adult psychopathology. J Nerv Ment Dis 2009; 197(1): 24-7.

[352] Wadsworth MEJ. Follow-up of the first national birth cohort: Findings from the Medical Research Council National Survey of Health and Development. Paediatr Perinat Epidemiol 1987; 1(1): 95-117.

[353] Wadsworth M, Maclean M, Kuh D, Rodgers B. Children of divorced and separated parents: summary and review of findings from a long-term follow-up study in the UK. Family Pract 1990; 7(2): 104-9.

[354] Rodgers B. Pathways between parental divorce and adult depression. J Child Psychol Psychiatry Allied Disciplines 1994; 35(7): 1289-308.

[355] Richards M, Hardy R, Wadsworth M. The effects of divorce and separation on mental health in a national UK birth cohort. Psychol Med 1997; 27(5): 1121-8.

[356] Chase-Lansdale PL, Cherlin AJ, Kiernan KE. The long-term effects of parental divorce on the mental health of young adults: a developmental perspective. Child Dev 1995; 66(6): 1614-34.

[357] Rodgers B, Power C, Hope S. Parental divorce and adult psychological distress: evidence from a national birth cohort: a research note. J Child Psychol Psychiatry Allied Disciplines 1997; 38(7):867-72

[358] Cherlin AJ, Chase-Lansdale PL, McRae C. Effects of parental divorce on mental health throughout the life course. Am Soc Rev 1998; 63(2): 239.

[359] Fergusson DM, Boden JM, Horwood LJ. Exposure to single parenthood in childhood and later mental health, educational, economic, and criminal behavior outcomes. Arch Gen Psychiatry 2007; 64(9): 1089-95.

[360] Thompson RG Jr., Lizardi D, Keyes KM, Hasin DS. Childhood or adolescent parental divorce/separation, parental history of alcohol problems, and offspring lifetime alcohol dependence. Drug Alcohol Depend 2008; 98(3): 264-9.
[361] Sauvola A, Miettunen J, Jarvelin MR, Rasanen P. An examination between single-parent family background and drunk driving in adulthood: findings from the Northern Finland 1966 Birth Cohort. Alcohol Clin Exp Res 2001; 25(2): 206-9.

[362] Sauvola A, Koskinen O, Jokelainen J, Hakko H, Jarvelin MR, Rasanen P. Family type and criminal behaviour of male offspring: The Northern Finland 1966 Birth Cohort Study. Int J Soc Psychiatry $2002 ; 48(2): 115-21$.

[363] Koskinen O, Sauvola A, Valonen P, Hakko H, Marjo-Riitta Jr, Rasanen $\mathrm{P}$. Increased risk of violent recidivism among adult males is related to single-parent family during childhood: The Northern Finland 1966 Birth Cohort Study. J Forensic Psychiatry 2001; 12(3): 539-48.

[364] Gilman SE, Kawachi I, Fitzmaurice GM, Buka SL. Family disruption in childhood and risk of adult depression. Am J Psychiatry 2003; 160(5): 939-46.

[365] Gilman SE, Kawachi I, Fitzmaurice GM, Buka L. Socio-economic status, family disruption and residential stability in childhood: relation to onset, recurrence and remission of major depression. Psychol Med 2003; 33(8): 1341-55.

[366] Spence SH, Najman JM, Bor W, O'Callaghan MJ, Williams GM. Maternal anxiety and depression, poverty and marital relationships factors during early childhood as predictors of anxiety and depressive symptoms in adolescence. J Child Psychol Psychiatry Allied Dis 2002; 43(4): 457-69.

[367] Pesonen AK, Raikkonen K, Heinonen K, Kajantie E, Forsen T, Eriksson JG. Depressive symptoms in adults separated from their parents as children: a natural experiment during World War II. Am J Epidemiol 2007; 166(10): 1126-33.

[368] Rusby JS, Tasker F. Long-term effects of the British evacuation of children during World War 2 on their adult mental health. Aging Ment Health 2009; 13(3): 391-404.

[369] Scott S, Spender Q, Doolan M, Jacobs B, Aspland H. Multicentre controlled trial of parenting groups for childhood antisocial behaviour in clinical practice. BMJ 2001; 323(7306): 194-8.

[370] Brugha TS, Morrell CJ, Slade P, Walters SJ. Universal prevention of depression in women postnatally: cluster randomized trial evidence in primary care. Psychol Med 2011; 41(4): 739-48.

[371] Solantaus T, Toikka S. The Effective Family Programme. Preventative Services for the Children of Mentally Ill Parents in Finland. Int J Mental Health Promotion 2006; 8(4): 37-44.

[372] Toikka S, Solantaus T. The Effective Family Programme II. Clinicians' Experiences of Training in Promotive and Preventative Methods in Child Mental Health. Int J Mental Health Promotion 2006; 8(4): 4-10.

[373] Beardslee WR, Gladstone TR, Wright EJ, Cooper AB. A familybased approach to the prevention of depressive symptoms in chil dren at risk: evidence of parental and child change. Pediatrics 2003 112(2): e119-e131.

[374] Patterson J, Mockford C, Stewart-Brown S. Parents' perceptions of the value of the Webster-Stratton Parenting Programme: a qualitative study of a general practice based initiative. Child Care Health Dev 2005; 31(1): 53-64.

(c) Fryers and Brugha; Licensee Bentham Open.

This is an open access article licensed under the terms of the Creative Commons Attribution Non-Commercial License (http://creativecommons.org/licenses/by-nc/3.0/) which permits unrestricted, non-commercial use, distribution and reproduction in any medium, provided the work is properly cited. 